\title{
Metal-Catalyzed Regioselective Oxy-Functionalization of Internal Alkynes: An Entry into Ketones, Acetals, and Spiroketals
}

\author{
Bo Liu and Jef K. De Brabander* \\ Department of Biochemistry, The University of Texas Southwestern Medical Center at Dallas, 5323 \\ Harry Hines Boulevard, Dallas, TX 75390-9038 \\ E-mail:jdebra@biochem.swmed.edu
}

Table of Contents:

1. General Techniques

2. Experimental Procedures

3. Characterization Data

4. Copies of GC Chromatograms

5. Copies of NMR Spectra ...p1

...p1

...p3

...p8

...p13

\section{General Techniques}

Unless otherwise noted, commercially available materials were used without further purification. $\mathrm{MeAuPPh}_{3}$ was prepared according to the literature [A. Tamaki, S. A. Magennis, J. K. Kochi, J. Am. Chem. Soc. 1974, 96, 6140-6148]. All solvents were of HPLC or ACS grade. Solvents used in metal-catalyzed reactions were distilled from drying reagents under a nitrogen atmosphere: $\mathrm{Et}_{2} \mathrm{O}$ and THF from sodium benzophenone ketyl.

Gas chromatography (GC) was performed on an HP 6890N autosampling GC with an HP-5 capillary column $(30.0 \mathrm{~m} \times 320 \mu \mathrm{m} \times 0.25 \mu \mathrm{m})$ and equipped with a FID detector.

Flash chromatography (FC) was performed using E Merck silica gel 60 (240-400 mesh) or alumina (80-200 mesh). Thin layer chromatography (TLC) was performed using precoated plates purchased from E. Merck (silica gel $60 \mathrm{PF} 254,0.25 \mathrm{~mm}$ ) that were visualized using $\mathrm{KMnO}_{4}$ or Ce (IV) stain.

Nuclear magnetic resonance (NMR) spectra were recorded on a Varian Inova-400 or Mercury-300 spectrometer at operating frequencies of 400/300 MHz ( ${ }^{1} \mathrm{H}$ NMR) or $100 / 75 \mathrm{MHz}\left({ }^{13} \mathrm{C} \mathrm{NMR}\right)$. Chemical shifts $(\delta)$ are given in ppm relative to residual solvent (usually chloroform $\delta 7.27$ for ${ }^{1} \mathrm{H}$ NMR or $\delta 76.9$ for proton decoupled ${ }^{13} \mathrm{C}$ NMR), and coupling constants $(J)$ in Hz. Multiplicity is tabulated as s for singlet, $d$ for doublet, $t$ for triplet, q for quadruplet, and $\mathrm{m}$ for multiplet, whereby the prefix app is applied in cases where the true multiplicity is unresolved, and $b r$ when the signal in question is broadened.

Infrared spectra were recorded on a Perkin-ElmerI 1000 series FTIR instrument, with wavenumbers expressed in $\mathrm{cm}^{-1}$, using samples prepared as thin films between salt plates. Low resolution mass spectra (LRMS) were recorded on a Shimadzu 2010-LCMS, or at the NIH regional mass spectrometry facility at the University of Washington, St. Louis, MO. High-resolution mass spectra (HRMS) were recorded at the NIH regional mass spectrometry facility at the University of Washington, St. Louis, MO.

\section{Experimental Procedures}

\section{General procedure for Table 1}

Under Ar, a solution of $\mathbf{1 b}(0.20 \mathrm{mmol}, 48.0 \mathrm{mg})$ in ether $(0.1 \mathrm{ml})$ or acetonitrile $(0.1 \mathrm{ml})$ was added dropwise to a solution of the catalyst in ether $(0.9 \mathrm{ml})$ or acetonitrile $(0.9 \mathrm{ml})$ at room temperature. The resultant solution was stirred at room temperature for the indicated time, followed by derivatization to spiroketals $\mathbf{2} / \mathbf{3}$ (Method A described below). 


\section{General procedure for Table 2}

Under Ar, a solution of the starting material $(0.20 \mathrm{mmol})$ in ether $(0.1 \mathrm{ml})$ was added dropwise to a solution of Zeise's dimer $(1 \mathrm{~mol} \%, 2.0 \mu \mathrm{mol}, 1.2 \mathrm{mg})$ in ether $(0.9 \mathrm{ml})$ at room temperature. The resultant solution was stirred at room temperature for $30 \mathrm{~min}$, followed by derivatization to spiroketals $\mathbf{2} / \mathbf{3}$ (Method A described below, entries 1-2),

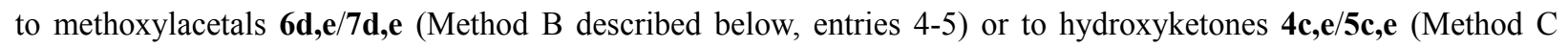
described below, entries 3 and 6 ).

\section{General procedure for Table 3, entries 1-7, 9-10:}

Under Ar, a solution of the starting material $(0.20 \mathrm{mmol})$ in ether $(0.1 \mathrm{ml})$ was added to a solution of Zeise's dimer $(1 \mathrm{~mol} \%, 2.0 \mu \mathrm{mol}, 1.2 \mathrm{mg})$ or $\mathrm{PdCl}_{2}\left(\mathrm{PhCN}_{2}(3 \mathrm{~mol} \%, 6 \mu \mathrm{mol}, 2.6 \mathrm{mg})\right.$ in ether $(0.9 \mathrm{ml})$ at room temperature. However, 4-nonyne-1,9-diol (entry 9) was added directly to a solution of $\mathrm{PdCl}_{2}\left(\mathrm{PhCN}_{2}\right.$ in ether (1 ml) in a glove bag since it cannot dissolve in ether. The resultant solution was stirred at room temperature for the indicated amount of time, followed by derivatization to spiroketals $\mathbf{2 / 3}$ (Method A described below, entries 1-3, 9), or to methoxylacetals 6d-g/9d-g (Method B described below, entries 4-7, 10).

\section{Procedure for Scheme 2 and Table 3, entry 8:}

A solution of the starting material $(0.20 \mathrm{mmol})$ in acetonitrile $(0.1 \mathrm{ml})$ was added to a solution of $\mathrm{PdCl}_{2}(1 \mathrm{~mol} \%$ or $5 \mathrm{~mol} \%)$ in acetonitrile $(0.9 \mathrm{ml})$ and water $(0.1 \mathrm{ml})$ under reflux. The resulting mixture was stirred for $30 \mathrm{~min}$ at reflux, followed by derivatization to spiroketals $\mathbf{2 / 3}$ (Method A described below, Eq. 1), or methoxylacetals 6e/9e (Method B described below, entry 8).

\section{General procedure for Table 3, entries 11-14:}

Under Ar, a suspension of $\mathrm{MeAuPPh}_{3}{ }^{1}(10 \mu \mathrm{mol}, 4.7 \mathrm{mg})$ and $\mathrm{AgPF}_{6}(10 \mu \mathrm{mol}, 2.5 \mathrm{mg})$ in isopropyl ether $(0.9$ $\mathrm{ml}$ ) was stirred at room temperature for $20 \mathrm{~min}$. Then a solution of the starting material $(0.2 \mathrm{mmol})$ in isopropyl ether $(0.1 \mathrm{ml})$ was added. However, 4-nonyne-1,9-diol (entry 11) was added directly to a suspension of $\mathrm{MeAuPPh}_{3}$ and $\mathrm{AgPF}_{6}$ in isopropyl ether $(1 \mathrm{ml})$ in a glove bag since it does not dissolve in isopropyl ether. The resultant mixture was stirred at room temperature for $13 \mathrm{~h}$, followed by derivatization to spiroketals $\mathbf{2} / \mathbf{3}$ (Method A described below, entries 11-12), or to methoxylacetals 6e,f/9e,f (Method B described below, entries 13-14).

\section{Derivatization method A:}

Camphorsulphonic acid $(5 \mathrm{~mol} \%, 10 \mu \mathrm{mol}, 2.3 \mathrm{mg})$, water $(30 \mu \mathrm{l})$ and acetonitrile $(1 \mathrm{ml})$ were added to the reaction mixture and the resulting solution was stirred at room temperature for $4 \mathrm{~h}$. Anhydrous $\mathrm{MgSO}_{4}(0.100 \mathrm{~g})$ was then added and the suspension was stirred at room temperature for $5 \mathrm{~h}$. $\mathrm{Et}_{3} \mathrm{~N}(50 \mu \mathrm{l})$ and hexadecane $(0.12 \mathrm{mmol}$, $26.9 \mathrm{mg}$; internal standard for GC analysis) were added to the suspension. An aliquot was removed, filtered through a short pad of alumina to remove the catalyst and then subjected to GC analysis to determine the yield and ratio (2:3).

GC conditions: initial temperature, $100{ }^{\circ} \mathrm{C}$; hold time, $1.50 \mathrm{~min}$; then $20{ }^{\circ} \mathrm{C} / \mathrm{min}$; final temperature $250{ }^{\circ} \mathrm{C}$. Retention times: $t_{R}(2)=3.18 \mathrm{~min} ; t_{R}(3)=3.37 \mathrm{~min} ; t_{R}$ (hexadecane) $=6.20 \mathrm{~min}$. The $\mathrm{GC}$ detector sensitivity corrections were obtained in separate experiments with known amounts of pure compound 2 (purchased from Alfa Aesar) and hexadecane (purchased from Aldrich); the relative sensitivity of hexadecane was 1.5 times that of compound 2 .

\section{Derivatization method B:}

Pyridinium $p$-toluenesulphonate $(5 \mathrm{~mol} \%, 10 \mu \mathrm{mol}, 2.5 \mathrm{mg})$, anhydrous $\mathrm{MeOH}(1.0 \mathrm{ml})$ and $\mathrm{HC}(\mathrm{OMe})_{3}(0.10$ $\mathrm{ml})$ were added to the reaction mixture. After $2 \mathrm{~h}, \mathrm{Et}_{3} \mathrm{~N}(50 \mu \mathrm{l})$ was added to quench the reaction. The solvents were evaporated in vacuo and the residue was passed through a short pad of alumina (eluted with EtOAc/hexanes $=1 / 8$ ) to remove the catalyst. After removal of the solvents, the residue was dissolved in dry and neutral $\mathrm{CDCl}_{3}(4 \AA \mathrm{MS}$ and $\left.\mathrm{K}_{2} \mathrm{CO}_{3}\right)$ to determine the yield and ratio $(\mathbf{6}: 7$, or $6: 9)$. 


\section{Derivatization method C:}

THF $(1 \mathrm{ml})$ and water $(4.0 \mathrm{mmol}, 20$ eq., $72 \mu \mathrm{l})$ was added to the reaction mixture. After $5 \mathrm{~min}, \mathrm{Et}_{3} \mathrm{~N}(50 \mu \mathrm{l})$ was added to quench the reaction. After evaporation of the solvents, the residue was purified by flash chromatography $\left(\mathrm{SiO}_{2}, \mathrm{EtOAc} /\right.$ hexanes $\left.=1 / 5\right)$ to give hydroxyketones $\mathbf{4}$ and $\mathbf{5}(\mathbf{4 c}$ and $\mathbf{5 c}$ are separable compounds by flash chromatography, whereas $4 \mathbf{e}$ and $\mathbf{5 e}$ are unseparable and their ratio was determined after derivatization to methylacetals $6 \mathbf{e} / 7 \mathbf{e}$ via method B.

\section{General procedure for Table 4:}

Under Ar, a solution of Zeise's dimer $(2 \mu \mathrm{mol}, 1.2 \mathrm{mg})$ in $\mathrm{MeOH}(1.0 \mathrm{mmol}, 40 \mu \mathrm{l})$ and $\mathrm{HC}(\mathrm{OMe})_{3}(50 \mu \mathrm{l})$ was added dropwise to a solution of the starting material $(0.2 \mathrm{mmol})$ and $\mathrm{HC}(\mathrm{OMe})_{3}(50 \mu \mathrm{l})$ in freshly distilled THF $(1.0$ $\mathrm{ml})$. After the resultant yellow solution was stirred at room temperature for $30 \mathrm{~min}$, triethylamine $(50 \mu \mathrm{l})$ was added to quench the reaction. The solution was concentrated and the residue was passed through a short pad of alumina eluted with mixed solvents $($ EtOAc/hexanes $=1 / 8)$ to remove the catalyst. After removing the solvents, the residue was dissolved in dry and neutral $\mathrm{CDCl}_{3}\left(4 \AA \mathrm{MS}\right.$ and $\left.\mathrm{K}_{2} \mathrm{CO}_{3}\right)$ to determine the yield and regioselectivity.

\section{Hydroalkoxylation of 4-pentyn-1-ol in the presence of $\mathrm{CD}_{3} \mathrm{OD}$ (Scheme 2)}

A solution of 4-pentyn-1-ol $(0.1 \mathrm{mmol}, 8.4 \mathrm{mg})$ in $\mathrm{CD}_{3} \mathrm{OD}(0.1 \mathrm{ml})$ was added to a solution of Zeise's dimer $(1 \mu \mathrm{mol}$, $0.6 \mathrm{mg})$ in $\mathrm{CD}_{3} \mathrm{OD}(0.4 \mathrm{ml})$ in a NMR tube at room temperature. The resulting solution was monitored by ${ }^{1} \mathrm{H}$ NMR.

\section{Characterization Data}

\section{4-Nonyne-1,9-diol (1a):}

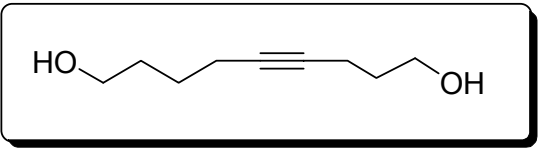

${ }^{1} \mathrm{H}$ NMR (400 MHz, $\left.\mathrm{CDCl}_{3}\right) \delta 3.76(2 \mathrm{H}, \mathrm{t}, J=6.1), 3.68(2 \mathrm{H}, \mathrm{t}, J=6.4)$, 2.22-2.30 (2H, m), 2.11-2.20 (2H, m), 2.00-2.20 (2H, -Oㅡ, br.), $1.74(2 \mathrm{H}$, quint., $J=6.5), 1.65-1.68(2 \mathrm{H}, \mathrm{m}), 1.56-1.60(2 \mathrm{H}, \mathrm{m}) ;{ }^{13} \mathrm{C} \mathrm{NMR}(100$ $\left.\mathrm{MHz}, \mathrm{CDCl}_{3}\right) \delta 80.5,79.7,62.1,61.5,31.6,31.3,25.0,18.3,15.2$; IR (thin film, $\left.\mathrm{cm}^{-1}\right) 3335,2940,2867,1434,1332,1057$; ESI-MS (m/z) calc. for $\mathrm{C}_{9} \mathrm{H}_{16} \mathrm{O}_{2} \mathrm{Na}\left([\mathrm{M}+\mathrm{Na}]^{+}\right) 179$, found 179.1 .

\section{9-(Tetrahydro-pyran-2-yloxy)-non-5-yn-1-ol (1b):}

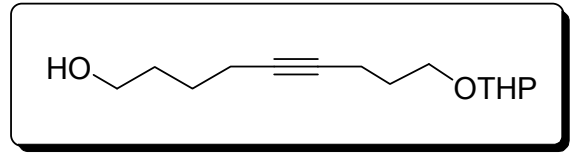

${ }^{1} \mathrm{H}$ NMR $\left(300 \mathrm{MHz}, \mathrm{CDCl}_{3}\right) \delta 4.62(1 \mathrm{H}, \mathrm{t}, J=3.8), 3.79-3.91(2 \mathrm{H}, \mathrm{m})$, $3.66(2 \mathrm{H}, \mathrm{t}, J=6.3), 3.44-3.55(2 \mathrm{H}, \mathrm{m}), 2.27(2 \mathrm{H}, \mathrm{tt}, J=7.0,2.2), 2.19$ $(2 \mathrm{H}, \mathrm{tt}, J=6.8,2.2), 1.48-1.95(12 \mathrm{H}, \mathrm{m}) ;{ }^{13} \mathrm{C} \mathrm{NMR}\left(75 \mathrm{MHz}, \mathrm{CDCl}_{3}\right) \delta$ 98.5, 80.1, 79.7, 65.8, 62.2, 61.9, 31.7, 30.5, 29.0, 25.3, 25.1, 19.2, 18.3, 15.4; IR (thin film, $\mathrm{cm}^{-1}$ ) 3418, 2942, 2869, 2361, 2341, 1454, 1441, 1137, 1120, 1062, 1034; ESI-MS (m/z) calc. for $\mathrm{C}_{14} \mathrm{H}_{24} \mathrm{O}_{3} \mathrm{Na}\left([\mathrm{M}+\mathrm{Na}]^{+}\right) 263$, found 263 .

\section{9-(tert-Butyl-dimethyl-silanyloxy)-non-5-yn-1-ol (1c):}

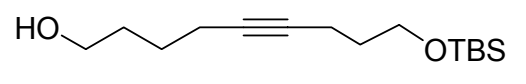

${ }^{1} \mathrm{H}$ NMR (300 MHz, $\left.\mathrm{CDCl}_{3}\right) \delta 3.69(4 \mathrm{H}, \mathrm{t}, J=6.1), 2.05-2.25(4 \mathrm{H}, \mathrm{m})$, 1.54-1.73 (6H, m), $0.90(9 \mathrm{H}, \mathrm{s}), 0.06(6 \mathrm{H}, \mathrm{s}) ;{ }^{13} \mathrm{C}$ NMR (100 MHz, $\left.\mathrm{CDCl}_{3}\right) \delta 80.0,79.8,62.3,61.6,32.0,31.7,25.8,25.2,18.4,18.2,15.0$, -5.4; IR (thin film, $\mathrm{cm}^{-1}$ ) 3350, 2930, 2858, 2361, 2173, 1255, 1104, 1068,

Acetic acid 9-hydroxy-non-4-ynyl ester (1d):

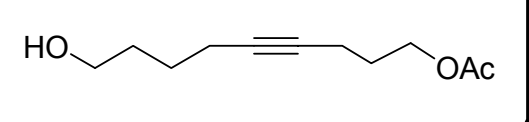

${ }^{1} \mathrm{H}$ NMR $\left(400 \mathrm{MHz}, \mathrm{CDCl}_{3}\right) \delta 4.16(2 \mathrm{H}, \mathrm{t}, J=6.4), 3.66(2 \mathrm{H}, \mathrm{t}, J=6.5)$, 2.22-2.27 (2H, m), 2.16-2.21 (2H, m), $2.05(3 \mathrm{H}, \mathrm{s}), 1.80(2 \mathrm{H}$, quint., $J=$ 6.6), 1.64-1.70 (2H, m), 1.54-1.60 (2H, m); $\left.{ }^{13} \mathrm{C} \mathrm{NMR} \mathrm{(100} \mathrm{MHz,} \mathrm{CDCl}_{3}\right) \delta$ 
171.1, 80.6, 78.8, 63.1, 62.3, 31.7, 27.8, 25.0, 20.8, 18.3, 15.2; IR (thin film, $\mathrm{cm}^{-1}$ ) 3415, 2940, 1740, 1368, 1245, 1045; ESI-MS (m/z) calc. for $\mathrm{C}_{11} \mathrm{H}_{18} \mathrm{O}_{3}\left([\mathrm{M}+\mathrm{Na}]^{+}\right)$221, found 221.0.

Dodec-5-yn-1-ol (1e): ${ }^{1}$

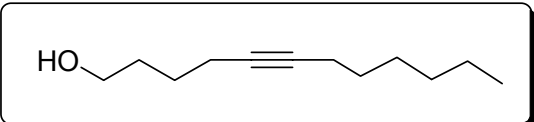

${ }^{1} \mathrm{H}$ NMR $\left(300 \mathrm{MHz}, \mathrm{CDCl}_{3}\right) \delta 3.68(2 \mathrm{H}, \mathrm{t}, J=6.4), 2.21(2 \mathrm{H}, \mathrm{tt}, J=6.7$, 2.4), 2.14 (2H, tt, $J=7.0,2.4), 1.19-1.74(12 \mathrm{H}, \mathrm{m}), 0.89$ (3H, t, $J=6.9$ );

${ }^{13} \mathrm{C}$ NMR $\left(75 \mathrm{MHz}, \mathrm{CDCl}_{3}\right) \delta 80.5,79.5,62.1,31.6,31.2,28.9,28.3,25.2$, 22.4, 18.5, 18.3, 13.8; IR (thin film, $\mathrm{cm}^{-1}$ ) 3341, 2931, 2859, 2360, 2341, 1456, 1060; ESI-MS (m/z) calc. for $\mathrm{C}_{12} \mathrm{H}_{23} \mathrm{O}\left([\mathrm{M}+\mathrm{H}]^{+}\right) 183$, found 183 .

\section{9-Methoxy-non-5-yn-1-ol:}

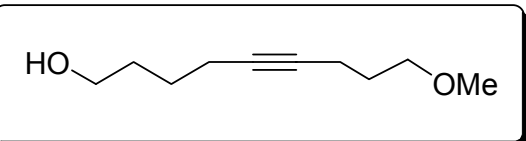

${ }^{1} \mathrm{H}$ NMR $\left(300 \mathrm{MHz}, \mathrm{CDCl}_{3}\right) \delta 3.67(2 \mathrm{H}, \mathrm{t}, J=6.4), 3.46(2 \mathrm{H}, \mathrm{t}, J=6.4)$, 3.34 (3H, s), 2.16-2.27 (4H, m), 1.46-1.79 (6H, m); ${ }^{13} \mathrm{C}$ NMR (100 MHz, $\left.\mathrm{CDCl}_{3}\right) \delta 80.0,79.6,71.2,62.2,58.4,31.7,28.8,25.1,18.3,15.3$; IR (thin film, $\left.\mathrm{cm}^{-1}\right) 3392,2935,2869,1450,1119,1066$; ESI-MS (m/z) calc. for

$\mathrm{C}_{10} \mathrm{H}_{19} \mathrm{O}_{2}\left([\mathrm{M}+\mathrm{H}]^{+}\right) 171$, found 171 .

\section{0-(tert-Butyl-dimethyl-silanyloxy)-dec-6-yn-2-ol:}

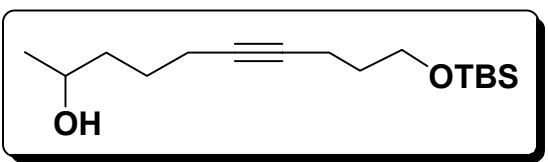

$\left.{ }^{1} \mathrm{H} \mathrm{NMR}\left(300 \mathrm{MHz}, \mathrm{CDCl}_{3}\right) \delta 3.76-3.88(1 \mathrm{H}, \mathrm{qt}, J=6.1,6.1)\right), 3.69(2 \mathrm{H}$, $\mathrm{t}, J=6.2), 2.12-2.30(4 \mathrm{H}, \mathrm{m}), 1.40-1.72(6 \mathrm{H}, \mathrm{m}), 1.21(3 \mathrm{H}, \mathrm{d}, J=6.1)$; ${ }^{13} \mathrm{C}$ NMR $\left(100 \mathrm{MHz}, \mathrm{CDCl}_{3}\right) \delta 79.9,79.8,67.4,61.6,38.2,31.9,25.8$, 25.1, 23.4, 18.5, 18.2, 14.9, -5.5; IR (thin film, $\mathrm{cm}^{-1}$ ) 3357, 2953, 2930, 2858, 2173, 1255, 1105, 1072, 836, 776; ESI-MS (m/z) calc. for $\mathrm{C}_{16} \mathrm{H}_{33} \mathrm{O}_{2} \mathrm{Si}\left([\mathrm{M}+\mathrm{H}]^{+}\right)$285, found 285 .

2-Methyl-tetradec-7-yn-3-ol:

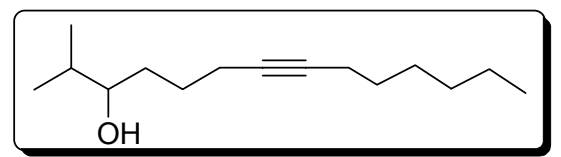

${ }^{1} \mathrm{H}$ NMR $\left(400 \mathrm{MHz}, \mathrm{CDCl}_{3}\right) \delta 3.33-3.38(1 \mathrm{H}, \mathrm{m}), 2.17(2 \mathrm{H}, \mathrm{tt}, J=6.6,2.4)$, $2.11(2 \mathrm{H}, \mathrm{tt}, J=7.0,2.4), 1.22-1.69(13 \mathrm{H}, \mathrm{m}), 0.90(3 \mathrm{H}, \mathrm{d}, J=2.8), 0.89$ $(3 \mathrm{H}, \mathrm{d}, J=2.8), 0.86(3 \mathrm{H}, \mathrm{t}, J=7.1) ;{ }^{13} \mathrm{C} \mathrm{NMR}\left(100 \mathrm{MHz}, \mathrm{CDCl}_{3}\right) \delta 80.5$, 79.7, 76.1, 33.4, 33.0, 31.2, 28.9, 28.4, 25.4, 22.4, 18.6 (2C), 17.0, 13.9; IR (thin film, $\mathrm{cm}^{-1}$ ) 3368, 2957, 2931, 2860, 1467, 1375 (d), 1131; ESI-MS (m/z) calc. for $\mathrm{C}_{15} \mathrm{H}_{29} \mathrm{O}\left([\mathrm{M}+\mathrm{H}]^{+}\right) 225$, found 225 .

\section{Dodec-6-yne-1,2-diol:}

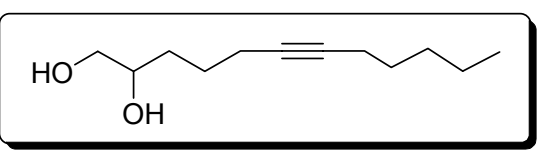

${ }^{1} \mathrm{H}$ NMR $\left(400 \mathrm{MHz}, \mathrm{CDCl}_{3}\right) \delta 3.73-3.79(1 \mathrm{H}, \mathrm{m}), 3.67(1 \mathrm{H}, \mathrm{A}$ of $\mathrm{AB}, \mathrm{dd}, J$ $=11.0,3.1), 3.46(1 \mathrm{H}, \mathrm{B}$ of $\mathrm{AB}, \mathrm{dd}, J=11.0,7.7), 2.10-2.26(4 \mathrm{H}, \mathrm{m})$, $1.42-1.73(6 \mathrm{H}, \mathrm{m}), 1.25-1.42(4 \mathrm{H}, \mathrm{m}), 0.90(3 \mathrm{H}, \mathrm{t}, J=6.0) ;{ }^{13} \mathrm{C} \mathrm{NMR}(100$ $\left.\mathrm{MHz}, \mathrm{CDCl}_{3}\right) \delta 80.6,79.3,71.7,66.5,31.9,30.9,28.6,25.0,22.0,18.5$ (2C), 13.8; IR (thin film, $\mathrm{cm}^{-1}$ ) 3352, 1378, 1332, 1102, 1062; ESI-MS (m/z) calc. for $\mathrm{C}_{12} \mathrm{H}_{22} \mathrm{O}_{2} \mathrm{Na}\left([\mathrm{M}+\mathrm{Na}]^{+}\right) 221$, found 221.1 .

\section{9-(Tetrahydro-pyran-2-yloxy)-non-4-yn-1-ol (8b): ${ }^{2}$}

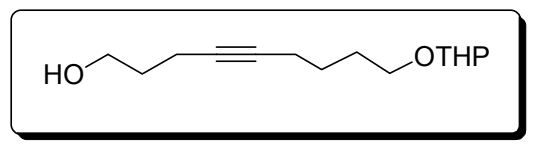

${ }^{1} \mathrm{H}$ NMR $\left(400 \mathrm{MHz}, \mathrm{CDCl}_{3}\right) \delta 4.59(1 \mathrm{H}, \mathrm{dd}, J=4.4,2.7), 3.76(2 \mathrm{H}, \mathrm{t}, J=$ 6.1), $3.76(1 \mathrm{H}, \mathrm{dt}, J=9.7,6.4), 3.73-3.79(1 \mathrm{H}, \mathrm{m}), 3.48-3.53(1 \mathrm{H}, \mathrm{m}), 3.41$ $(1 \mathrm{H}, \mathrm{dt}, J=9.7,6.4), 2.21-2.30(2 \mathrm{H}, \mathrm{m}), 2.17-2.20(2 \mathrm{H}, \mathrm{m}), 1.48-1.82(12 \mathrm{H}$, $\mathrm{m}) ;{ }^{13} \mathrm{CNMR}\left(100 \mathrm{MHz}, \mathrm{CDCl}_{3}\right) \delta 98.6,80.3,79.4,66.8,62.0,61.4,31.4,30.5,28.6,25.5,25.2,19.3,18.3,15.1 ; \mathrm{IR}$

\footnotetext{
${ }^{1}$ Known Compound, see: J. K. Stille, James H. Simpson, J. Am. Chem. Soc. 1987, 109, 2138-2152.

2 Known Compound, see: M. L. Sharma, S. Verma, T. Chand, Indian J. Chem. Sect.B 1995, 34, 1030-1034.
} 
(thin film, $\mathrm{cm}^{-1}$ ) 3409, 1453, 1440, 1352, 1323, 1284, 1261, 1201, 1186, 1157, 1137, 1120, 1075, 1034; ESI-MS (m/z) calc. for $\mathrm{C}_{14} \mathrm{H}_{24} \mathrm{O}_{3} \mathrm{Na}\left([\mathrm{M}+\mathrm{Na}]^{+}\right)$263, found 263.1.

\section{9-(tert-Butyl-dimethyl-silanyloxy)-non-4-yn-1-ol (8c):}

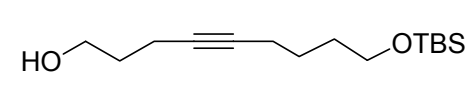

${ }^{1} \mathrm{H}$ NMR $\left(300 \mathrm{MHz}, \mathrm{CDCl}_{3}\right) \delta 3.75(2 \mathrm{H}, \mathrm{td}, J=5.8,5.8), 3.62(2 \mathrm{H}, \mathrm{t}, J=6.1)$, 2.24-2.30 (2H, m), 2.14-2.20 (2H, m), 1.73 (2H, quint., $J=6.1), 1.41-1.62$ $(5 \mathrm{H}, \mathrm{m}), 0.89(9 \mathrm{H}, \mathrm{s}), 0.04(6 \mathrm{H}, \mathrm{s}) ;{ }^{13} \mathrm{C} \mathrm{NMR}\left(75 \mathrm{MHz}, \mathrm{CDCl}_{3}\right) \delta 80.8,79.4$, 62.6, 62.0, 31.9, 31.5, 25.8, 25.3, 18.4, 18.2, 15.3, -5.4; IR (thin film, $\mathrm{cm}^{-1}$ ) $3352,1472,1255,1104$; ESI-MS (m/z) calc. for $\mathrm{C}_{15} \mathrm{H}_{31} \mathrm{O}_{2} \mathrm{Si}\left([\mathrm{M}+\mathrm{H}]^{+}\right) 271$, found 271; calc. for $\mathrm{C}_{15} \mathrm{H}_{30} \mathrm{O}_{2} \mathrm{SiNa}$ $\left([\mathrm{M}+\mathrm{Na}]^{+}\right)$293, found 293.1.

\section{Acetic acid 9-hydroxy-non-5-ynyl ester (8d):}

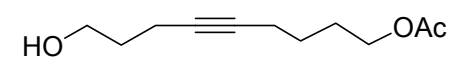

${ }^{1} \mathrm{H}$ NMR $\left(400 \mathrm{MHz}, \mathrm{CDCl}_{3}\right) \delta 4.08(2 \mathrm{H}, \mathrm{t}, J=6.6), 3.75(2 \mathrm{H}, \mathrm{t}, J=6.0)$, 2.20-2.29 (2H, m), 2.17-2.20 (2H, m), 2,05 (3H, s), 1.68-1.80 (4H, m), $1.50-1.60(2 \mathrm{H}, \mathrm{m}) ;{ }^{13} \mathrm{C} \mathrm{NMR}\left(100 \mathrm{MHz}, \mathrm{CDCl}_{3}\right) \delta 171.1,79.9,79.8,63.9$, 61.5, 31.4, 27.5, 25.2, 20.8, 18.2, 15.1; IR (thin film, $\mathrm{cm}^{-1}$ ) 3418, 2945, 2870, 1738, 1243, 1053; ESI-MS (m/z) calc. for $\mathrm{C}_{11} \mathrm{H}_{19} \mathrm{O}_{3}\left([\mathrm{M}+\mathrm{H}]^{+}\right) 199$, found 199.1; $\mathrm{C}_{11} \mathrm{H}_{18} \mathrm{O}_{3} \mathrm{Na}\left([\mathrm{M}+\mathrm{Na}]^{+}\right)$221, found 221.1.

Undec-4-yn-1-ol (8e): ${ }^{3}$

$$
\text { HO }={ }^{\mathrm{n}} \mathrm{C}_{6} \mathrm{H}_{13}
$$

${ }^{1} \mathrm{H}$ NMR (400 MHz, $\left.\mathrm{CDCl}_{3}\right) \delta 3.77$ (2H, br. s), 2.26-2.69 (2H, m), 2.11-2.17 $(2 \mathrm{H}, \mathrm{m}), 1.75(2 \mathrm{H}$, quint., $J=6.1), 1.21-1.51(8 \mathrm{H}, \mathrm{m}), 0.89(3 \mathrm{H}, \mathrm{t}, J=6.9) ;{ }^{13} \mathrm{C}$ NMR (75 MHz, $\left.\mathrm{CDCl}_{3}\right) \delta 80.9,79.1,61.8,31.4,31.2,28.9,28.4,22.4,18.5$, 15.2, 13.9; IR (thin film, $\mathrm{cm}^{-1}$ ) 3340, 2930, 2858, 1467, 1434, 1057; ESI-MS (m/z) calc. for $\mathrm{C}_{11} \mathrm{H}_{21} \mathrm{O}\left([\mathrm{M}+\mathrm{H}]^{+}\right) 168$, found 169.1 .

\section{9-Methoxymethoxy-non-4-yn-1-ol (8f):}

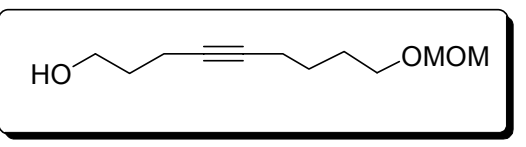

${ }^{1} \mathrm{H}$ NMR (400 MHz, $\left.\mathrm{CDCl}_{3}\right) \delta 4.63(2 \mathrm{H}, \mathrm{s}), 3.77(2 \mathrm{H}, \mathrm{t}, J=6.1), 3.55(2 \mathrm{H}, \mathrm{t}$, $J=6.4), 3.37(3 \mathrm{H}, \mathrm{s}), 2.22-2.30(2 \mathrm{H}, \mathrm{m}), 2.17-2.2 .22(2 \mathrm{H}, \mathrm{m}), 1.62-1.80(4 \mathrm{H}$, $\mathrm{m}), 1.52-1.62(2 \mathrm{H}, \mathrm{m}) ;{ }^{13} \mathrm{C} \mathrm{NMR}\left(100 \mathrm{MHz}, \mathrm{CDCl}_{3}\right) \delta 96.1,80.3,79.5,67.1$, 61.6, 54.9, 31.4, 28.7, 25.5, 18.3, 15.1; IR (thin film, $\mathrm{cm}^{-1}$ ) 3430, 1148, 1112, 1040; ESI-MS (m/z) calc. for $\mathrm{C}_{11} \mathrm{H}_{20} \mathrm{O}_{3} \mathrm{Na}\left([\mathrm{M}+\mathrm{Na}]^{+}\right) 200$, found 223.1.

9-Methoxy-non-4-yn-1-ol (8g):

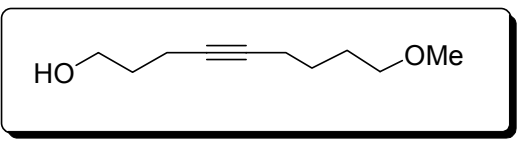

${ }^{1} \mathrm{H}$ NMR $\left(300 \mathrm{MHz}, \mathrm{CDCl}_{3}\right) \delta 3.75(2 \mathrm{H}, \mathrm{t}, J=6.0), 3.39(2 \mathrm{H}, \mathrm{t}, J=6.4)$, $3.33(3 \mathrm{H}, \mathrm{s}), 2.25-2.30(2 \mathrm{H}, \mathrm{m}), 2.15-2.20(2 \mathrm{H}, \mathrm{m}), 1.73(2 \mathrm{H}$, quint., $J=6.6)$, 1.63-1.68 (2H, m), 1.52-1.58 (2H, m); ${ }^{13} \mathrm{C}$ NMR $\left(75 \mathrm{MHz}, \mathrm{CDCl}_{3}\right) \delta 80.2$, 79.5, 72.1, 61.4, 58.3, 31.4, 28.5, 25.4, 18.3, 15.1; IR (thin film, $\mathrm{cm}^{-1}$ ) 3391, 1206, 1177, 1119, 1059; ESI-MS (m/z) calc. for $\mathrm{C}_{10} \mathrm{H}_{19} \mathrm{O}_{2}\left([\mathrm{M}+\mathrm{H}]^{+}\right)$171, found 171.1; calc. for $\mathrm{C}_{10} \mathrm{H}_{18} \mathrm{O}_{2} \mathrm{Na}\left([\mathrm{M}+\mathrm{Na}]^{+}\right)$193, found 193.1.

\section{2-Methoxy-2-[4-(tetrahydro-pyran-2-yloxy)-butyl]-tetrahydro-pyran:}

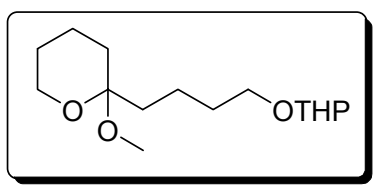

${ }^{1} \mathrm{H}$ NMR (400 MHz, CD $\left.{ }_{3} \mathrm{OD}\right) \delta 4.56(1 \mathrm{H}, \mathrm{t}, J=3.8), 3.85(1 \mathrm{H}, b r . \mathrm{t}), 3.73(1 \mathrm{H}, \mathrm{dt}, J=$ 9.6, 6.7), 3.58-3.66 (2H, m), 3.40-3.51 (1H, m), $3.37(1 \mathrm{H}, \mathrm{dt}, J=9.6,6.7), 3.16(3 \mathrm{H}, \mathrm{s})$, $1.25-1.85(18 \mathrm{H}, \mathrm{m}) ;{ }^{13} \mathrm{C} \mathrm{NMR}\left(100 \mathrm{MHz}, \mathrm{CDCl}_{3}\right) \delta 98.8,98.7,67.2,62.1,61.1,47.1$, 36.1, 32.6, 30.6, 29.8, 25.3, 25.1, 19.8, 19.5, 18.5; IR (thin film, $\mathrm{cm}^{-1}$ ) 1200, 1136, 1120, 1101, 1061, 1032; ESI-MS (m/z) calc. for $\mathrm{C}_{15} \mathrm{H}_{28} \mathrm{O}_{4} \mathrm{Na}\left([\mathrm{M}+\mathrm{Na}]^{+}\right)$295, found 295.

\footnotetext{
${ }^{3}$ Known Compound, see: K. M. Miller, T. F. Jamison, J. Am. Chem. Soc. 2004, 126, 15342-15343.
} 
tert-Butyl-[4-(2-methoxy-tetrahydro-pyran-2-yl)-butoxy]-dimethyl-silane:

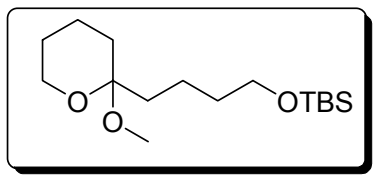

271.2093, found 271.2087.

${ }^{1} \mathrm{H}$ NMR $\left(300 \mathrm{MHz}, \mathrm{CDCl}_{3}\right) \delta 3.60(2 \mathrm{H}, \mathrm{t}, J=6.5), 3.55-3.68(2 \mathrm{H}, \mathrm{m}), 3.16(3 \mathrm{H}, \mathrm{s})$, 1.26-1.82 (12H), $0.88(9 \mathrm{H}, \mathrm{s}), 0.03(6 \mathrm{H}, \mathrm{s}) ;{ }^{13} \mathrm{C} \mathrm{NMR}\left(100 \mathrm{MHz}, \mathrm{CDCl}_{3}\right) \delta 98.8,62.7$, 61.1, 47.1, 36.0, 32.9, 32.6, 25.8, 25.1, 19.3, 18.5, 18.2, -5.4; IR (thin film, $\mathrm{cm}^{-1}$ ) 1255, 1101, 1071, 1061, 1030, 836, 775; EI-HRMS (m/z) calc. for $\mathrm{C}_{15} \mathrm{H}_{31} \mathrm{SiO}_{2}\left([\mathrm{M}-\mathrm{MeO}]^{+}\right)$

\section{2-Heptyl-2-methoxy-tetrahydro-pyran:}

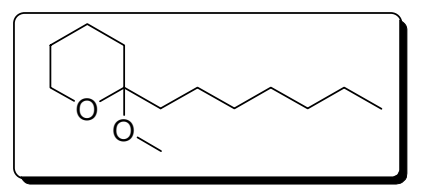

${ }^{1} \mathrm{H}$ NMR $\left(400 \mathrm{MHz}, \mathrm{CDCl}_{3}\right) \delta 3.58-3.67(2 \mathrm{H}, \mathrm{m}), 3.17(3 \mathrm{H}, \mathrm{s}), 1.23-1.81(18 \mathrm{H}, \mathrm{m})$, $0.88(3 \mathrm{H}, \mathrm{t}, J=6.8) ;{ }^{13} \mathrm{C} \mathrm{NMR}\left(100 \mathrm{MHz}, \mathrm{CDCl}_{3}\right) \delta 98.9,61.1,47.1,36.3,32.7,31.7$, 29.8, 29.1, 25.2, 23.1, 22.5, 18.5, 14.0; IR (thin film, $\mathrm{cm}^{-1}$ ) 1716, 1102, 1061, 1036; EI-HRMS (m/z) calc. for $\mathrm{C}_{13} \mathrm{H}_{26} \mathrm{O}_{2}\left(\mathrm{M}^{+}\right)$214.1933, found 214.1935.

\section{2-Methoxy-2-(4-methoxy-butyl)-tetrahydro-pyran: ${ }^{4}$}

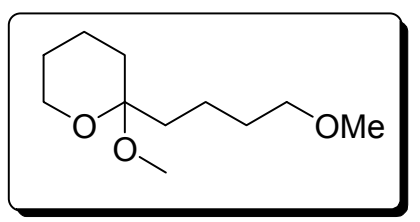

${ }^{1} \mathrm{H}$ NMR $\left(400 \mathrm{MHz}, \mathrm{CDCl}_{3}\right) \delta 3.56-3.67(2 \mathrm{H}, \mathrm{m}), 3.38(2 \mathrm{H}, \mathrm{t}, J=6.6), 3.33(3 \mathrm{H}, \mathrm{s})$, $3.17(3 \mathrm{H}, \mathrm{s}), 1.30-1.85(12 \mathrm{H}, \mathrm{m}) ;{ }^{13} \mathrm{C} \mathrm{NMR}\left(100 \mathrm{MHz}, \mathrm{CDCl}_{3}\right) \delta$ 98.7, 72.5, 61.1, 58.4, 47.1, 36.1, 32.6, 29.7, 25.1, 19.8, 18.5; IR (thin film, $\mathrm{cm}^{-1}$ ) 1215, 1193, 1179, $1119,1101,1060,1044,1028,882$; EI-HRMS (m/z) calc. for $\mathrm{C}_{11} \mathrm{H}_{22} \mathrm{O}_{3}\left(\mathrm{M}^{+}\right)$ 202.1569 , found 202.1565 .

Acetic acid 4-(2-methoxy-tetrahydro-pyran-2-yl)-butyl ester:

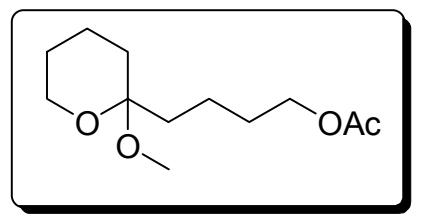

${ }^{1} \mathrm{H}$ NMR (400 MHz, $\left.\mathrm{CD}_{3} \mathrm{OD}\right) \delta 4.07$ (2H, t, $\left.J=6.7\right), 3.60-3.65(2 \mathrm{H}, \mathrm{m}), 3.17(3 \mathrm{H}, \mathrm{s})$, $2.05(3 \mathrm{H}, \mathrm{s}), 1.30-1.83(12 \mathrm{H}, \mathrm{m}) ;{ }^{13} \mathrm{C} \mathrm{NMR}\left(100 \mathrm{MHz}, \mathrm{CDCl}_{3}\right) \delta$ 171.0, 98.6, 64.2, 61.1, 47.1, 35.8, 32.5, 28.6, 25.0, 20.8, 19.6, 18.4; IR (thin film, $\mathrm{cm}^{-1}$ ) 1740, 1245, $1100,1060,1030$.

tert-Butyl-[4-(2-methoxy-6-methyl-tetrahydro-pyran-2-yl)-butoxy]-dimethyl-silane:

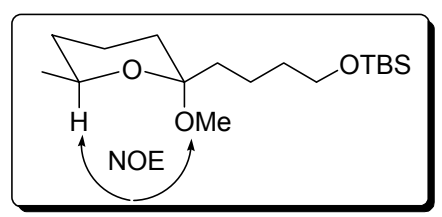

${ }^{1} \mathrm{H}$ NMR $\left(300 \mathrm{MHz}, \mathrm{CDCl}_{3}\right) \delta$ 3.62-3.72 ((1H, m), $3.60(2 \mathrm{H}, \mathrm{t}, J=6.3), 3.14(3 \mathrm{H}$, s), 1.10-1.85 (12H, m), $1.13(3 \mathrm{H}, \mathrm{d}, J=6.2), 0.88(9 \mathrm{H}, \mathrm{s}), 0.03(6 \mathrm{H}, \mathrm{s}) ;{ }^{13} \mathrm{C}$ NMR $\left(75 \mathrm{MHz}, \mathrm{CDCl}_{3}\right) \delta 99.4,66.0,62.7,47.0,36.2,32.9,32.5,32.1,26.0,25.8,21.7$, 19.3, 18.8, -5.4; IR (thin film, $\mathrm{cm}^{-1}$ ) 1255, 1093, 1055, 1031, 836, 775; EI-HRMS $(\mathrm{m} / \mathrm{z})$ calc. for $\mathrm{C}_{16} \mathrm{H}_{33} \mathrm{SiO}_{2}\left([\mathrm{M}-\mathrm{MeO}]^{+}\right)$285.2250, found 285.2259.

\section{2-Heptyl-6-isopropyl-2-methoxy-tetrahydro-pyran:}

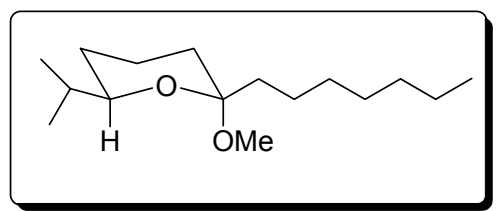

225.2218, found 225.2204.
${ }^{1} \mathrm{H} \mathrm{NMR}\left(400 \mathrm{MHz}, \mathrm{CDCl}_{3}\right) \delta 3.22(1 \mathrm{H}, \mathrm{ddd}, J=11.5,6.6,1.6), 3.15(3 \mathrm{H}, \mathrm{s})$, 1.09-1.82 (19H, m), 0.95 (3H, d, $J=6.8), 0.89$ (3H, d, $J=6.6$ ), 0.88 (3H, t, $J=$ 7.0); ${ }^{13} \mathrm{C}$ NMR (100 MHz, $\left.\mathrm{CDCl}_{3}\right) \delta 99.3,74.7,47.0,36.5,33.0,32.5,31.7$, 29.8, 29.1, 27.5, 23.2, 22.6, 18.9, 18.5, 14.0; IR (thin film, $\mathrm{cm}^{-1}$ ) 1375 (d), 1223, 1107, 1059, 1030, 945; EI-HRMS (m/z) calc. for $\mathrm{C}_{15} \mathrm{H}_{29} \mathrm{O}\left([\mathrm{M}-\mathrm{MeO}]^{+}\right)$

\footnotetext{
${ }^{4}$ For a known structural analog, see: G. Sauve, D. A. Schwartz, L. Ruest, P. Deslongchamps, Can. J. Chem. 1984, 62, 2929-2935.
} 


\section{1-Hydroxy-dodecan-5-one: ${ }^{5}$}

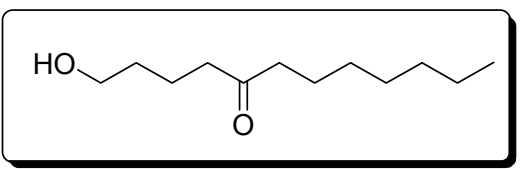

${ }^{1} \mathrm{H}$ NMR (400 MHz, $\left.\mathrm{CDCl}_{3}\right) \delta 3.62(2 \mathrm{H}, \mathrm{t}, J=6.2), 2.45(2 \mathrm{H}, \mathrm{t}, J=7.0)$, $2.39(2 \mathrm{H}, \mathrm{t}, J=7.4), 1.60-1.68(2 \mathrm{H}, \mathrm{m}), 1.53-1.60(4 \mathrm{H}, \mathrm{m}), 1.20-1.33(8 \mathrm{H}, b r$. s), $0.87(2 \mathrm{H}, \mathrm{t}, J=6.8) ;{ }^{13} \mathrm{C} \mathrm{NMR}\left(100 \mathrm{MHz}, \mathrm{CDCl}_{3}\right) \delta 211.6,62.0,42.7$, $42.1,31.9,31.5,29.0,28.9,23.7,22.4,19.5,13.9$.

1-(tert-Butyl-dimethyl-silanyloxy)-9-hydroxy-nonan-5-one:

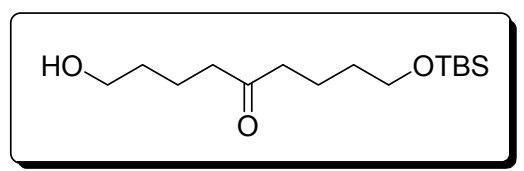

${ }^{1} \mathrm{H} \mathrm{NMR}\left(300 \mathrm{MHz}, \mathrm{CDCl}_{3}\right) \delta 3.62(3 \mathrm{H}, \mathrm{t}, J=6.1), 3.60(3 \mathrm{H}, \mathrm{t}, J=6.1)$, $2.45(2 \mathrm{H}, \mathrm{t}, J=6.7), 2.43(2 \mathrm{H}, \mathrm{t}, J=6.7), 1.43-1.69(8 \mathrm{H}, \mathrm{m}), 0.88(9 \mathrm{H}, \mathrm{s})$, $0.04(6 \mathrm{H}, \mathrm{s}) ;{ }^{13} \mathrm{C} \mathrm{NMR}\left(75 \mathrm{MHz}, \mathrm{CDCl}_{3}\right) \delta 211.2,62.7,62.1,42.4,42.1$, 32.1, 32.0, 25.8, 25.3, 20.2, 19.5, -5.5; IR (thin film, $\mathrm{cm}^{-1}$ ) 3216, 1713, 1255, 1194, 1100, 836, 776; ESI-MS (m/z) calc. for $\mathrm{C}_{15} \mathrm{H}_{32} \mathrm{O}_{3} \mathrm{SiNa}\left([\mathrm{M}+\mathrm{Na}]^{+}\right) 311.2$, found 311.1.

\section{5-Hexyl-6,8-dioxa-bicyclo[3.2.1]octane:}

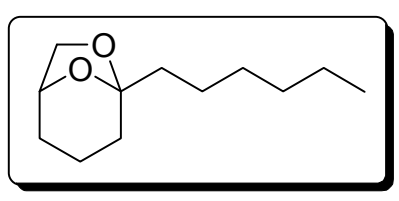

${ }^{1} \mathrm{H} \mathrm{NMR}\left(300 \mathrm{MHz}, \mathrm{CDCl}_{3}\right) \delta 4.50(1 \mathrm{H}, \mathrm{d}, J=3.4), 3.91(1 \mathrm{H}, \mathrm{d}, J=7.0), 3.81(1 \mathrm{H}, \mathrm{d}$, $J=6.0), 1.72-1.95(2 \mathrm{H}, \mathrm{m}), 1.50-1.71(5 \mathrm{H}, \mathrm{m}), 1.20-1.50(9 \mathrm{H}, \mathrm{m}), 0.81(3 \mathrm{H}, \mathrm{t}, J=$ 7.0); ${ }^{13} \mathrm{C}$ NMR (100 MHz, $\left.\mathrm{CDCl}_{3}\right) \delta 108.8,74.7,68.8,37.6,33.7,31.7,29.4,28.3$, 23.0, 22.4, 16.7, 14.0; IR (thin film, $\mathrm{cm}^{-1}$ ) 1239, 1174, 1074, 1019, 906, 851;

EI-HRMS (m/z) calc. for $\mathrm{C}_{12} \mathrm{H}_{22} \mathrm{O}_{2}\left(\mathrm{M}^{+}\right)$198.1620, found 198.1630 .

\section{2-Hexyl-2-methoxy-tetrahydro-furan:}

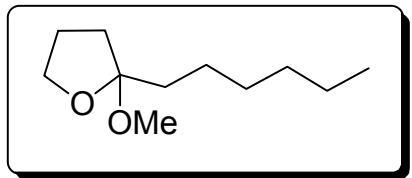

9d:

${ }^{1} \mathrm{H}$ NMR (400 MHz, CD3OD) $\delta 4.05$ (2H, t, $\left.J=6.4\right), 3.84-3.89(2 \mathrm{H}, \mathrm{m}), 3.16(3 \mathrm{H}, \mathrm{s}), 2.04(3 \mathrm{H}, \mathrm{s}), 1.28-2.04(12 \mathrm{H}$, $\mathrm{m})$.

9f:

${ }^{1} \mathrm{H}$ NMR (400 MHz, CD3OD) $\delta 4.62(2 \mathrm{H}, \mathrm{s}), 3.83-3.92(2 \mathrm{H}, \mathrm{m}), 3.52(2 \mathrm{H}, \mathrm{t}, J=6.4), 3.36(3 \mathrm{H}, \mathrm{s}), 3.16(3 \mathrm{H}, \mathrm{s})$, $1.36-2.08(12 \mathrm{H}, \mathrm{m})$.

${ }^{1} \mathrm{H}$ NMR $\left(300 \mathrm{MHz}, \mathrm{CDCl}_{3}\right) \delta 3.82-3.94(2 \mathrm{H}, \mathrm{m}), 3.18(3 \mathrm{H}, \mathrm{s}), 1.23-2.12(14 \mathrm{H}, \mathrm{m})$, $0.89(3 \mathrm{H}, \mathrm{t}, J=6.7) ;{ }^{13} \mathrm{C} \mathrm{NMR}\left(75 \mathrm{MHz}, \mathrm{CDCl}_{3}\right) \delta 109.4,67.1,47.9,35.3,34.1$, 31.7, 29.4, 24.6, 24.1, 22.5, 13.9; IR (thin film, $\mathrm{cm}^{-1}$ ) 1152, 1089, 1042; EI-HRMS $(\mathrm{m} / \mathrm{z})$ calc. for $\mathrm{C}_{11} \mathrm{H}_{22} \mathrm{O}_{2}\left(\mathrm{M}^{+}\right)$186.1620, found 186.1607 .

\footnotetext{
${ }^{5}$ Known compound, see: J. E. Stok, C. Lang, B. D. Schwartz, M. T. Fletcher, W. Kitching, J. J. De Voss, Org. Lett. 2001, $3,397-400$.
} 


\section{Copies of GC Chromatograms}

GC spectrum for commercial 1,7-dioxa-spiro[5.5] undecane (purchased from Alfa Aesar, $\mathrm{t}_{\mathrm{R}}=3.20 \mathrm{~min}$ ) and hexadecane (purchased from Aldrich, $\mathrm{t}_{\mathrm{R}}=6.21 \mathrm{~min}$ ).

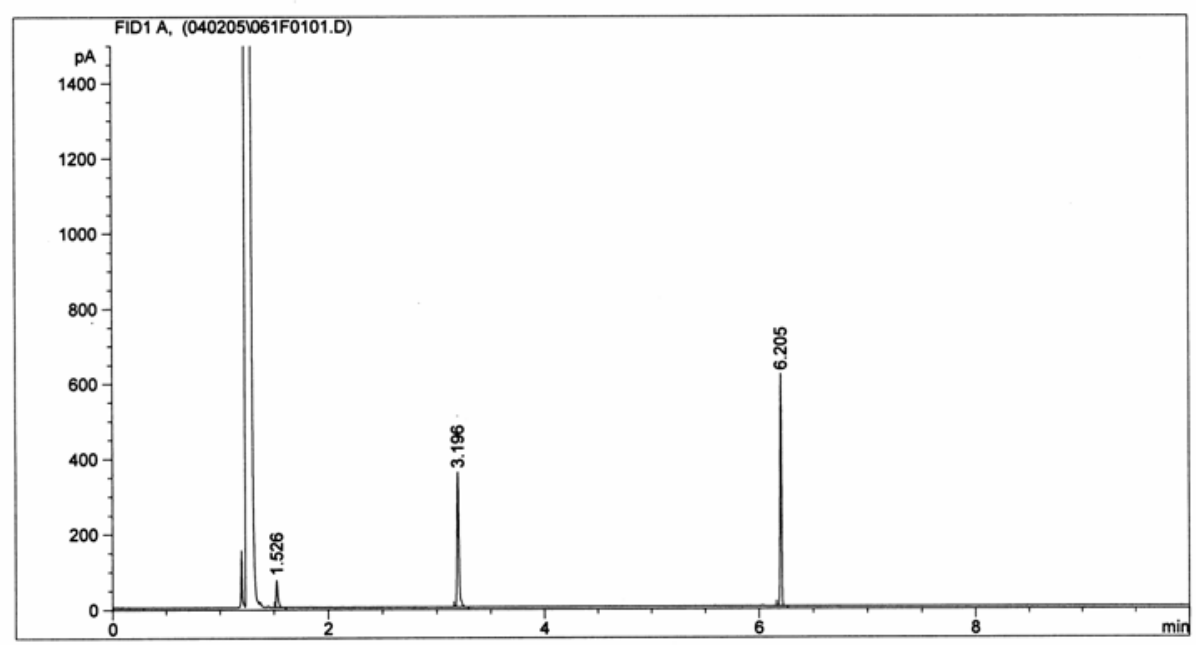

For GC detector, the relative sensitivity of hexadecane $(\mathrm{mg})$ to compound $2(\mathrm{mg})$ was determined to be 1.5 times. A correction curve was constructed, see the following chart.

W(IS)/W(2): weight (mg) of hexadecane (internal standard)/weight (mg) of compound 2.

PA(IS)/PA(2): peak area (GC) of hexadecane (internal standard)/peak area (GC) of compound 2.

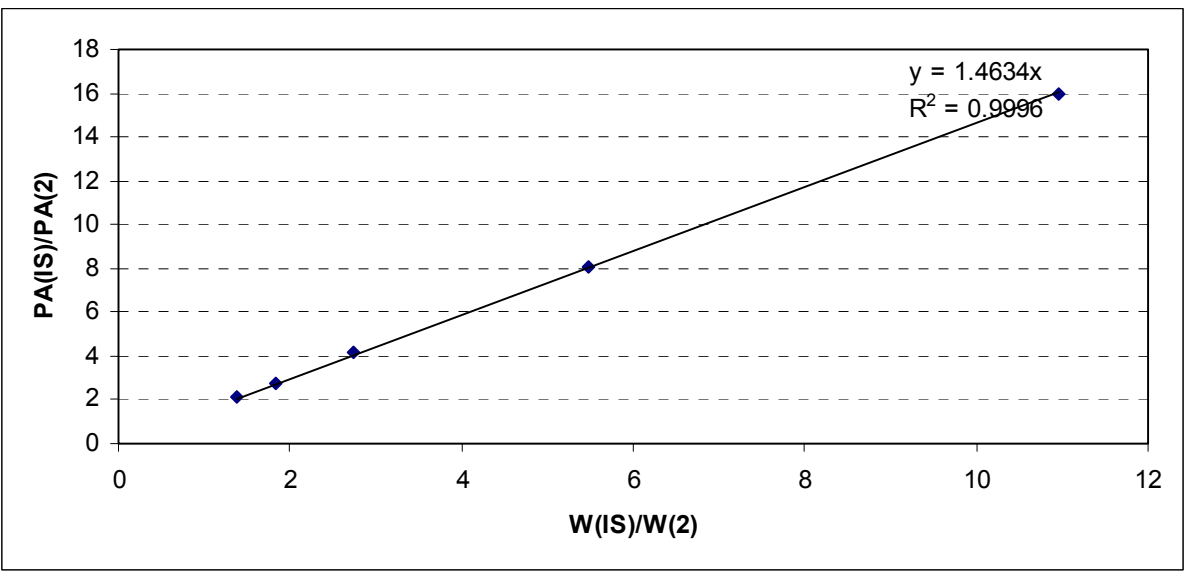


GC-chromatogram for Table 1, Entry $2(40 \%$ yield; $\mathbf{2 : 3}=1.3: 1)$
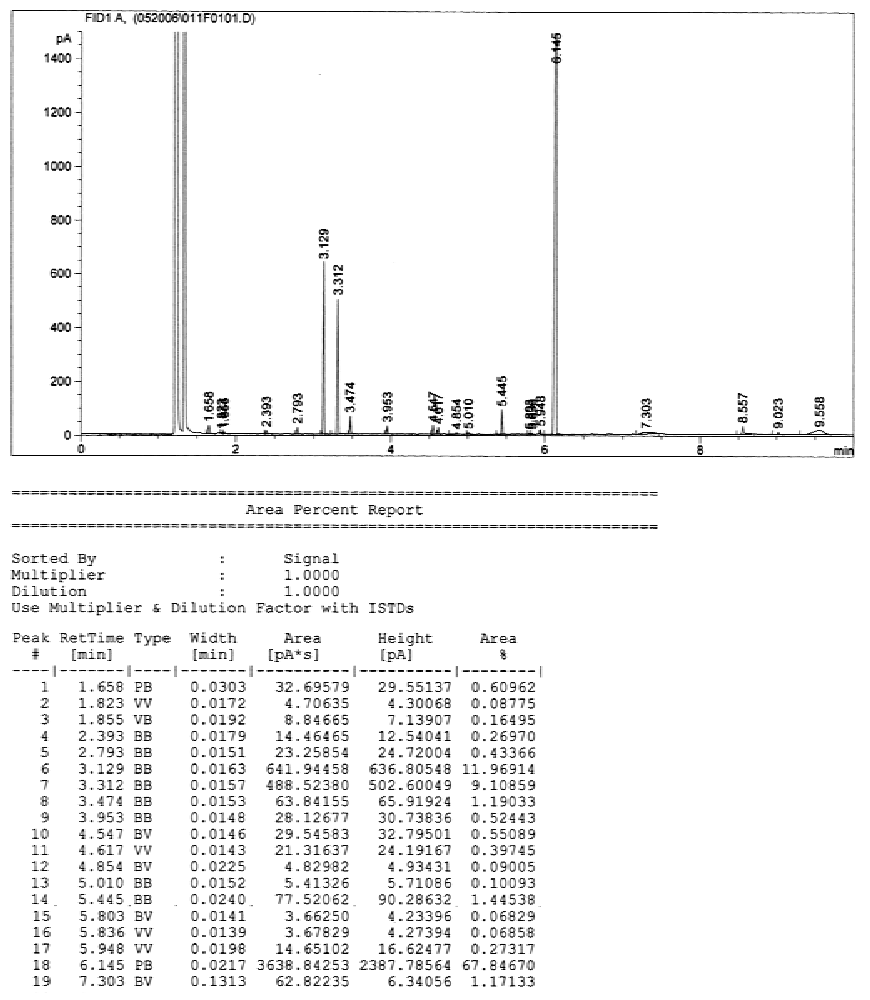

Table 1, Entry 6 (75\% yield; 2:3 = 30:1)

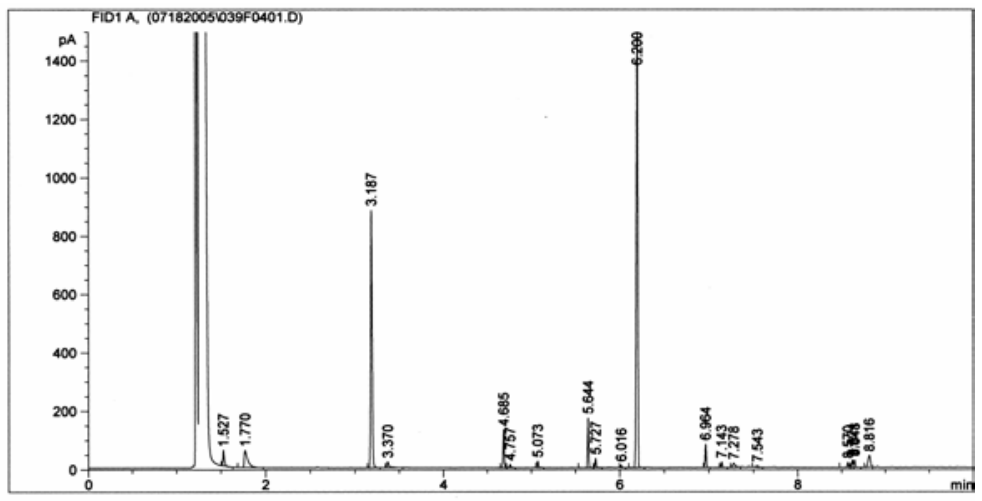

Area Percent Report

-

$\begin{array}{lll}\text { Sorted By } & : & \text { Signal } \\ \text { Multiplier } & : & 1.0000\end{array}$

Dilution
Use Multiplier \& Dilution Factor with ISTDs

Signa1 1: FID1 A,

\begin{tabular}{|c|c|c|c|c|c|c|}
\hline Peak & $\begin{array}{c}\text { RetTime } \\
{[\min ]}\end{array}$ & Type & $\begin{array}{l}\text { Width } \\
\text { [min] }\end{array}$ & $\begin{array}{r}\text { Area } \\
{\left[\mathrm{pA}^{*} \mathrm{~s}\right]}\end{array}$ & $\begin{array}{l}\text { Height } \\
\text { [pA] }\end{array}$ & $\underset{8}{\text { Area }}$ \\
\hline 1 & 1.527 & & 0.0347 & 55.85313 & 52.58298 & 1.49763 \\
\hline & & & 0.0367 & 153.11302 & 58.29697 & \\
\hline 3 & 3.187 & BB & 0.0186 & 1033.74927 & 883.51062 & 27.71862 \\
\hline 4 & 3.370 & BB & 0.0250 & 34.28918 & 19.85841 & 0.91942 \\
\hline 5 & 4.685 & BV & 0.0240 & 122.10622 & 129.95186 & 3.27412 \\
\hline 6 & & vB & 0.0246 & 12.28024 & 11.54994 & 0.32928 \\
\hline 7 & 5.073 & VB & 0.0280 & 21.49557 & 22.08447 & 0.57638 \\
\hline 8 & 5.644 & BV & 0.0225 & 148.08958 & 170.42004 & 3.97083 \\
\hline & 5.727 & vv & 0.0204 & 29.03759 & 31.44391 & 0.77860 \\
\hline 10 & 6.016 & VB & 0.0141 & 7.26048 & 8.26293 & 0.19468 \\
\hline 11 & 6.200 & BV & 0.02 & 1840.39722 & 1645.6 & 49.34 \\
\hline 12 & 6.964 & BB & 0.0281 & 72.56137 & 80.0 & 1.94564 \\
\hline 13 & 7.143 & BB & 0.0267 & 17.01611 & 19.02157 & \\
\hline 14 & 7.278 & BV & 0.0281 & 28.29582 & 14.53555 & 0.75872 \\
\hline 15 & 7.543 & vv & 0.03 & 10.32021 & 4.81643 & 0.27672 \\
\hline 16 & 8.570 & BV & 0.0162 & 16.59859 & 17.10408 & 0.44507 \\
\hline 17 & 8.621 & vv & 0.0202 & 25.62124 & 25.22815 & 0.68700 \\
\hline 18 & 8.648 & vB & 0.0171 & 25.44183 & 24.83167 & 0.68219 \\
\hline
\end{tabular}


GC-chromatogram for Table 2, Entry 1 (75\% yield; 2:3 30:1)
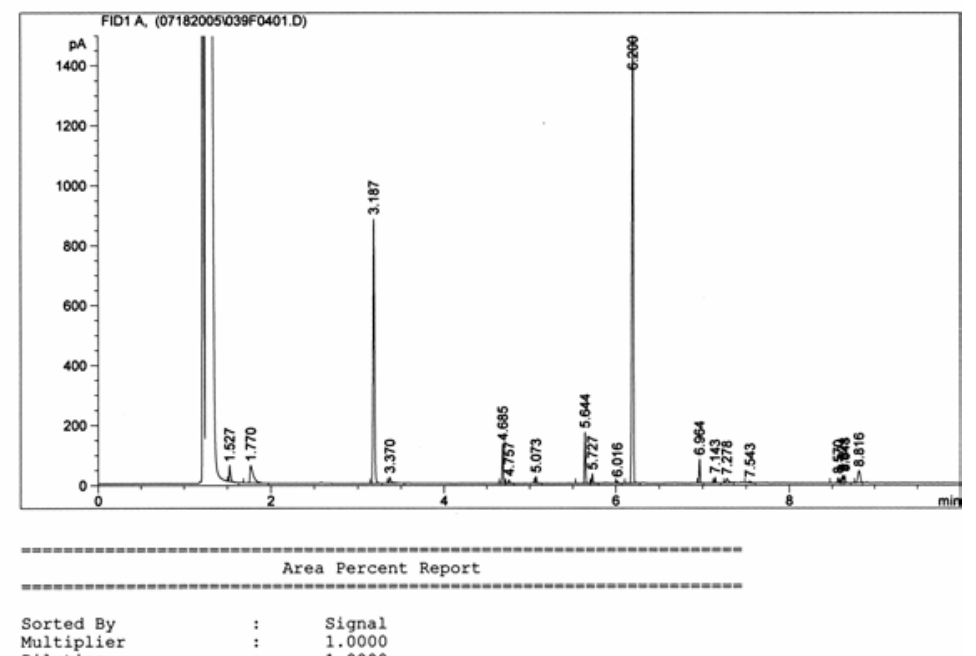

Dilution $\quad: \quad 1.0000$

Use Multiplier \& Dilution Factor with ISTDs

Signal 1: FID1 A,

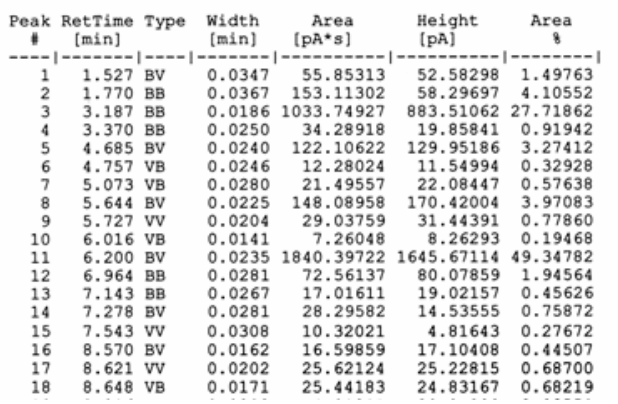

GC-chromatogram for Table 2, Entry 2 ( $83 \%$ yield; $\mathbf{2 : 3}=20: 1)$

Lash chanyed : 0/19/4UUq 1:19:00 rm Dy JmI
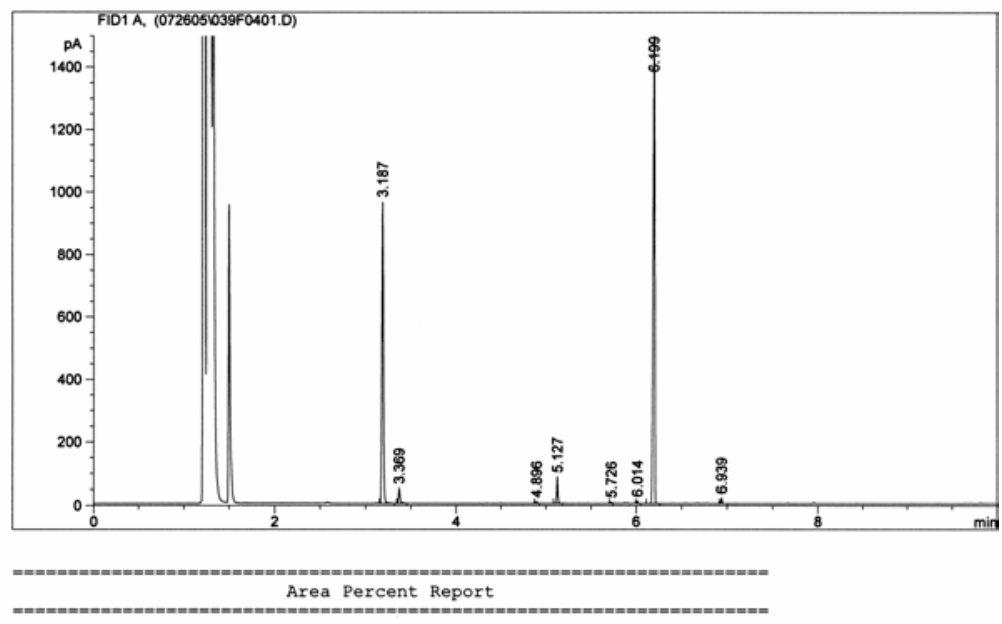

$\begin{array}{lll}\text { Sorted By } & : & \text { Signal } \\ \text { Multiplier } & : & 1.0000\end{array}$

Dilution $\quad 1.0000$

Use Multiplier \& Dilution Factor with ISTDs

Signal 1: FID1 A,

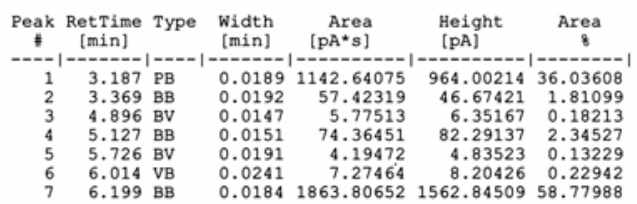


GC-chromatogram for Table 3, Entry 1 (70\% yield; $2: 3=11: 1)$
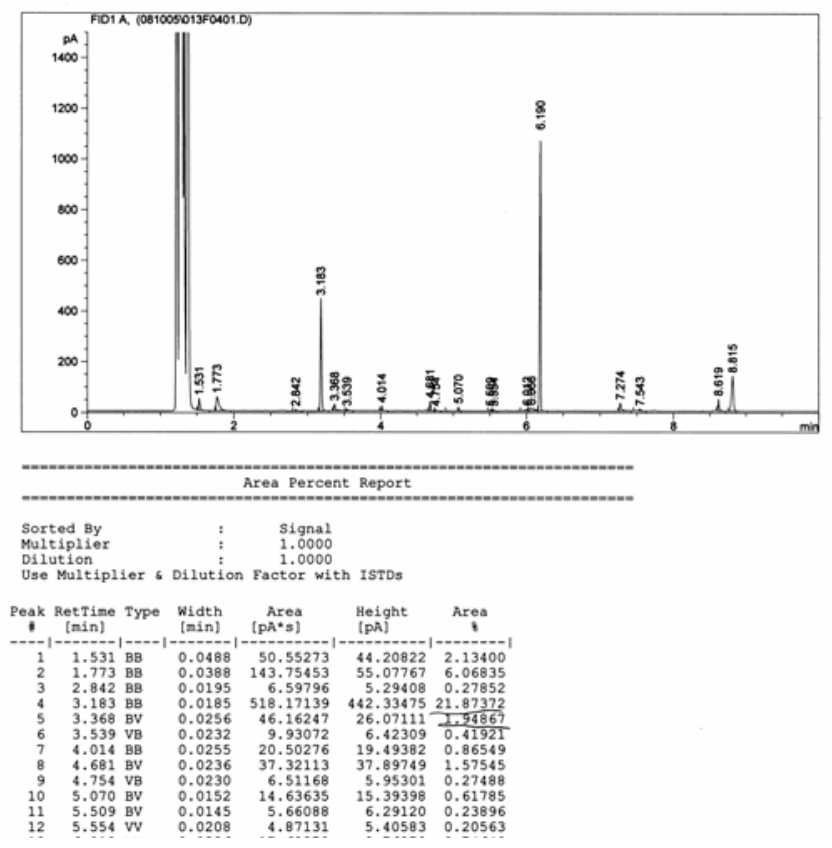

GC-chromatogram for Table 3, Entry 2 (60\% yield; 2:3 9:1)

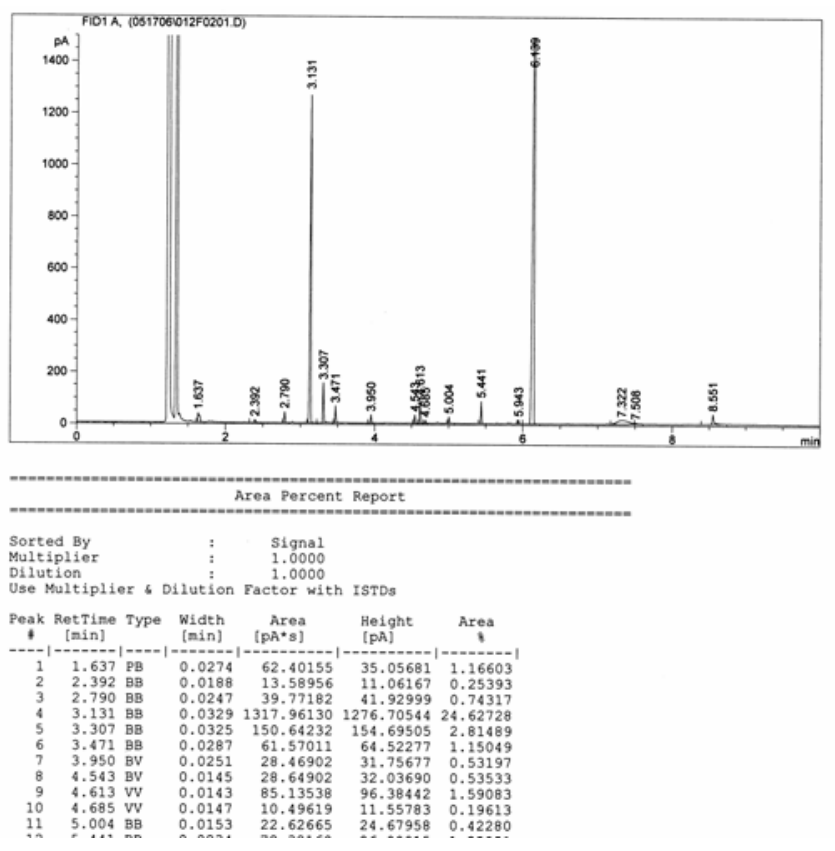


GC-chromatogram for Table 3, Entry 12 (73\% yield; 2:3 = 1:6.6)

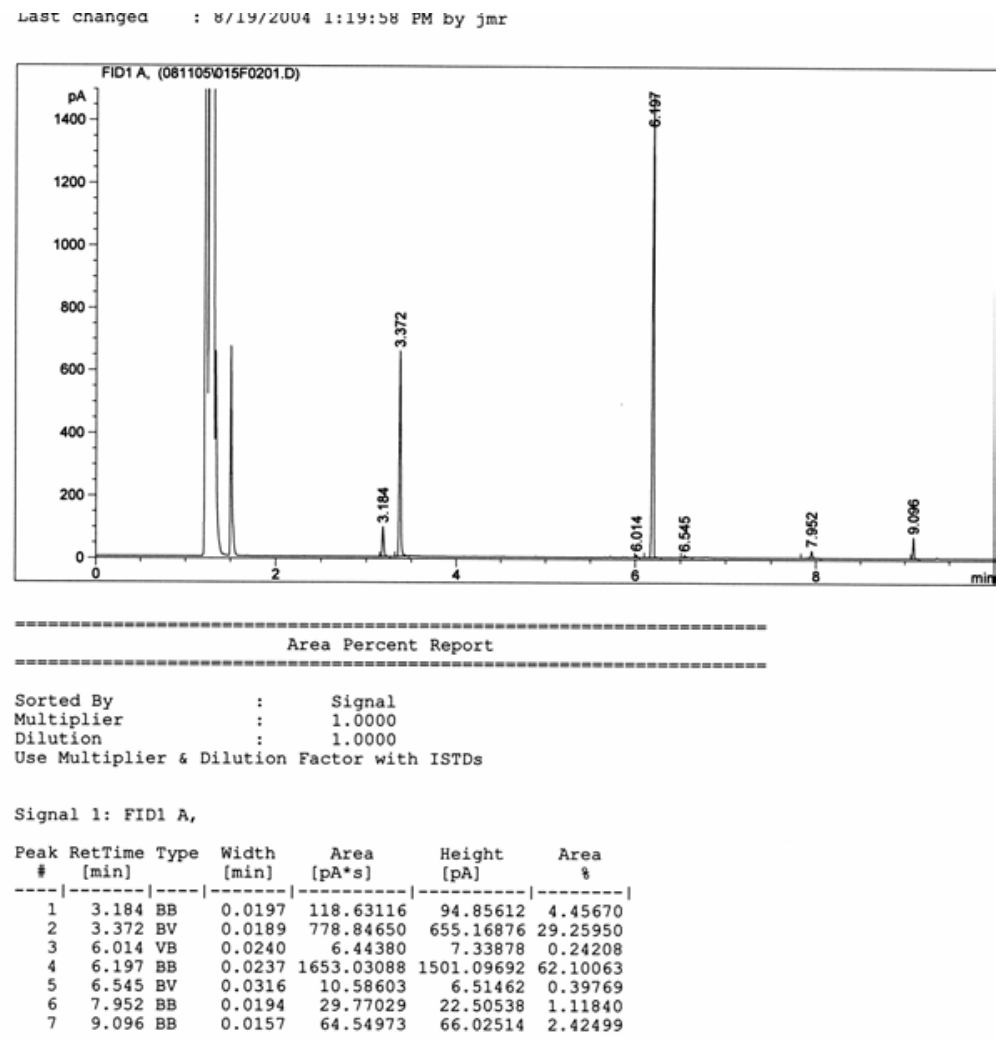




\section{Copies of NMR Spectra}

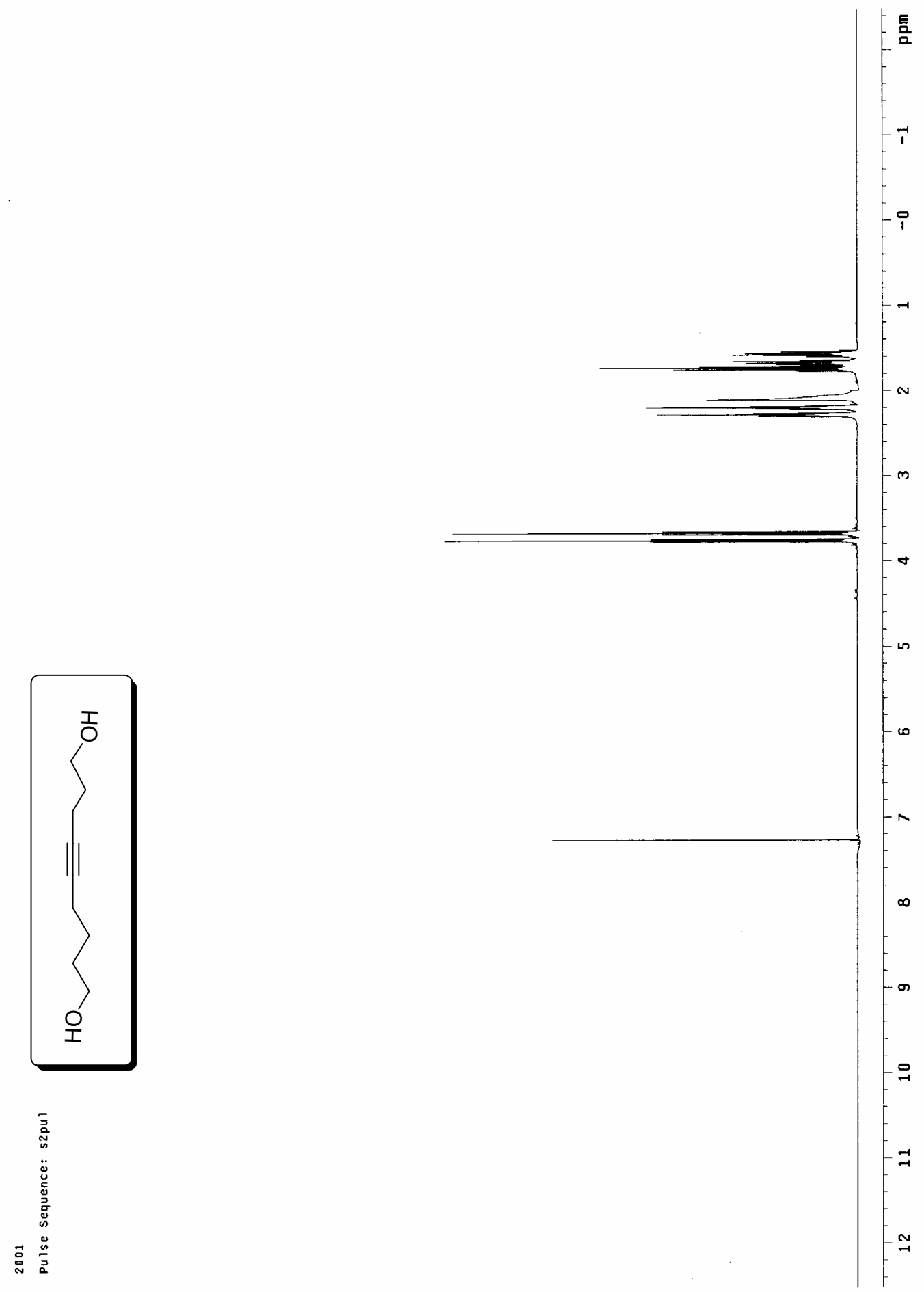




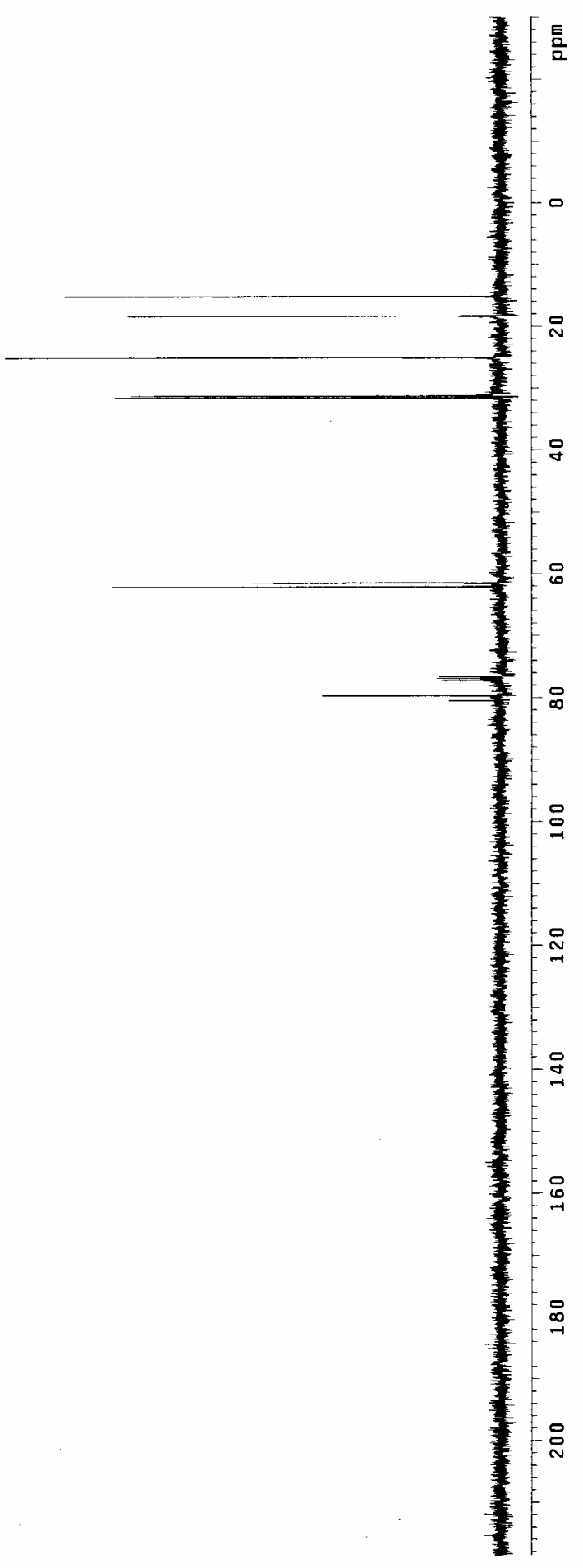




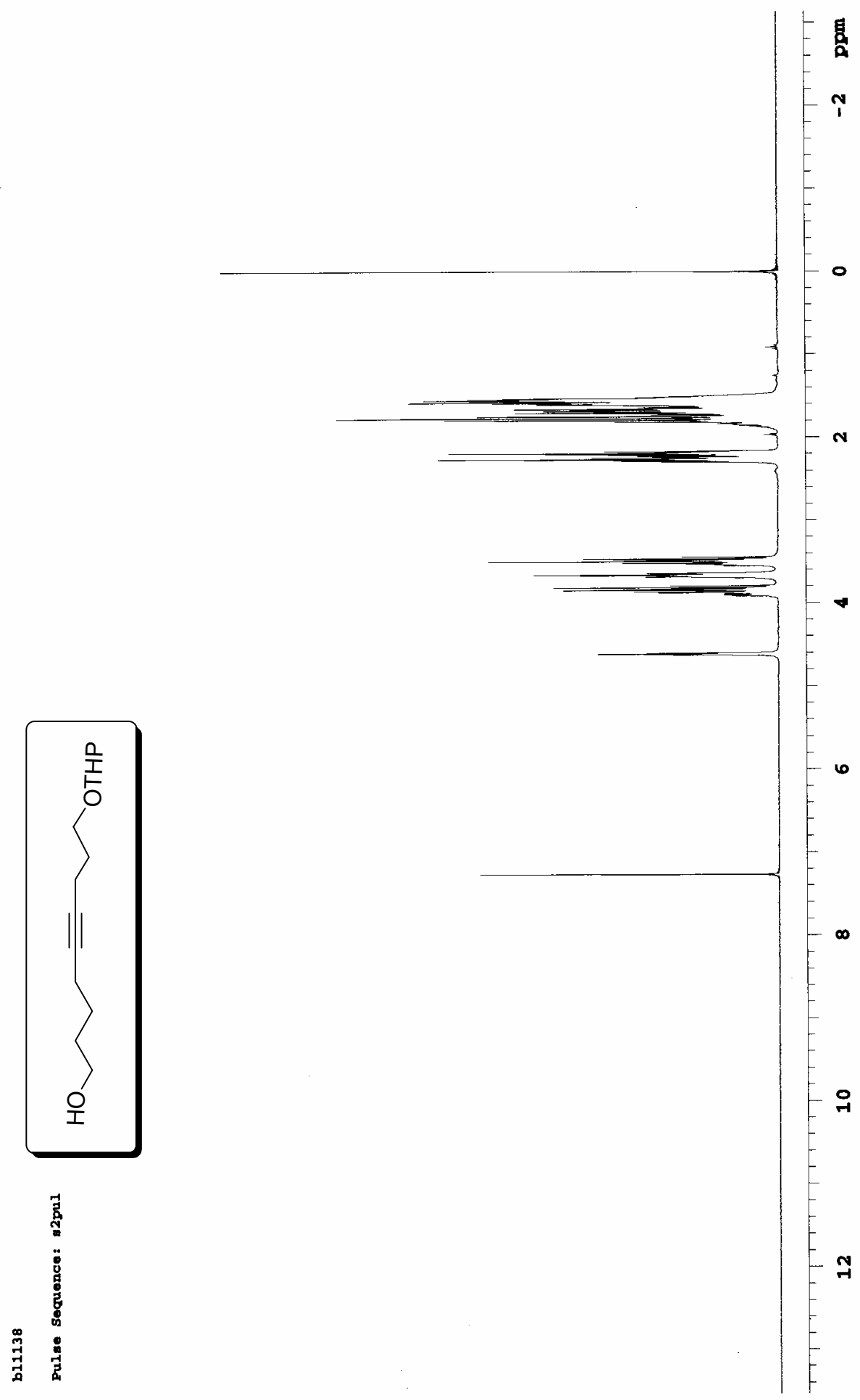

-15 - 


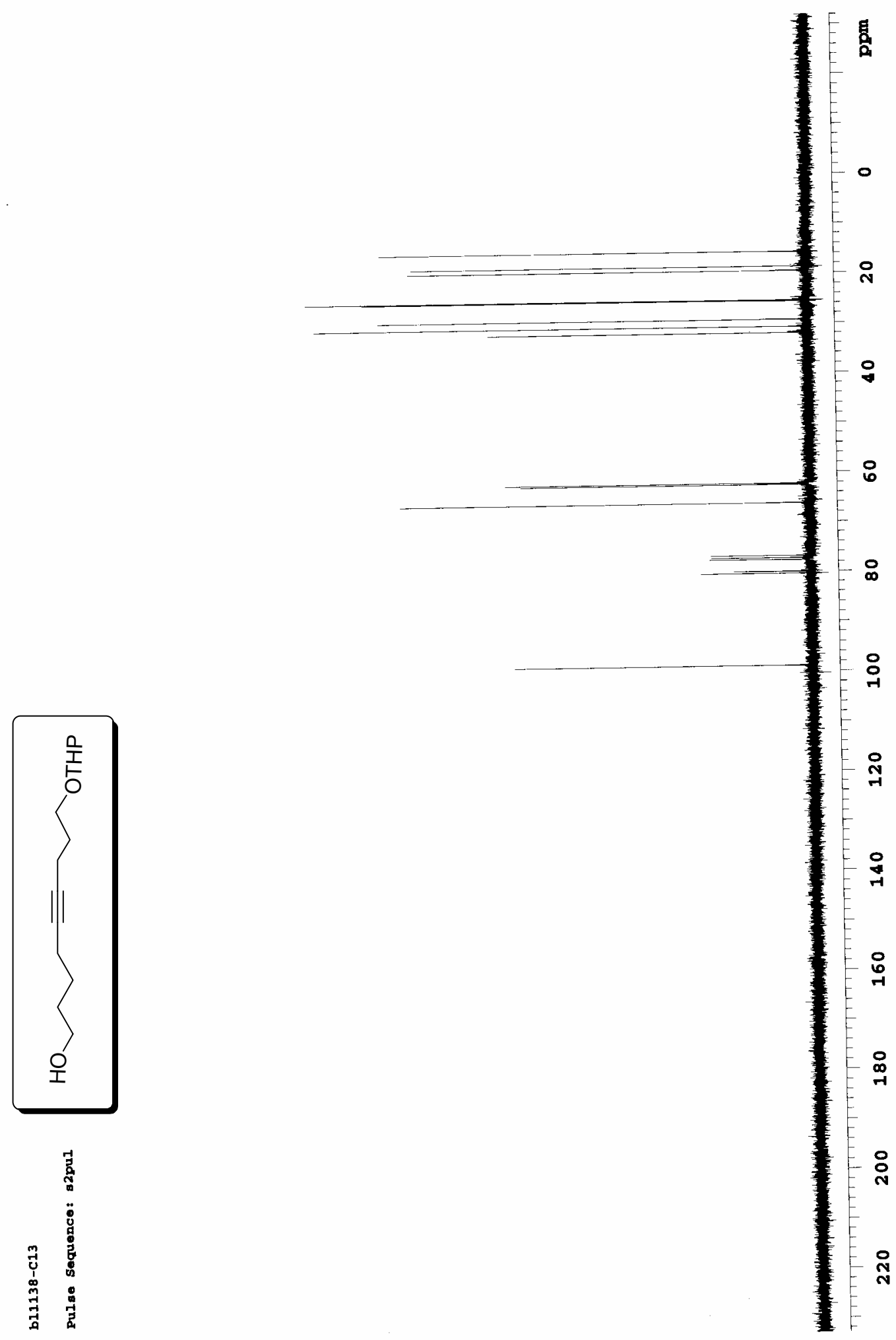




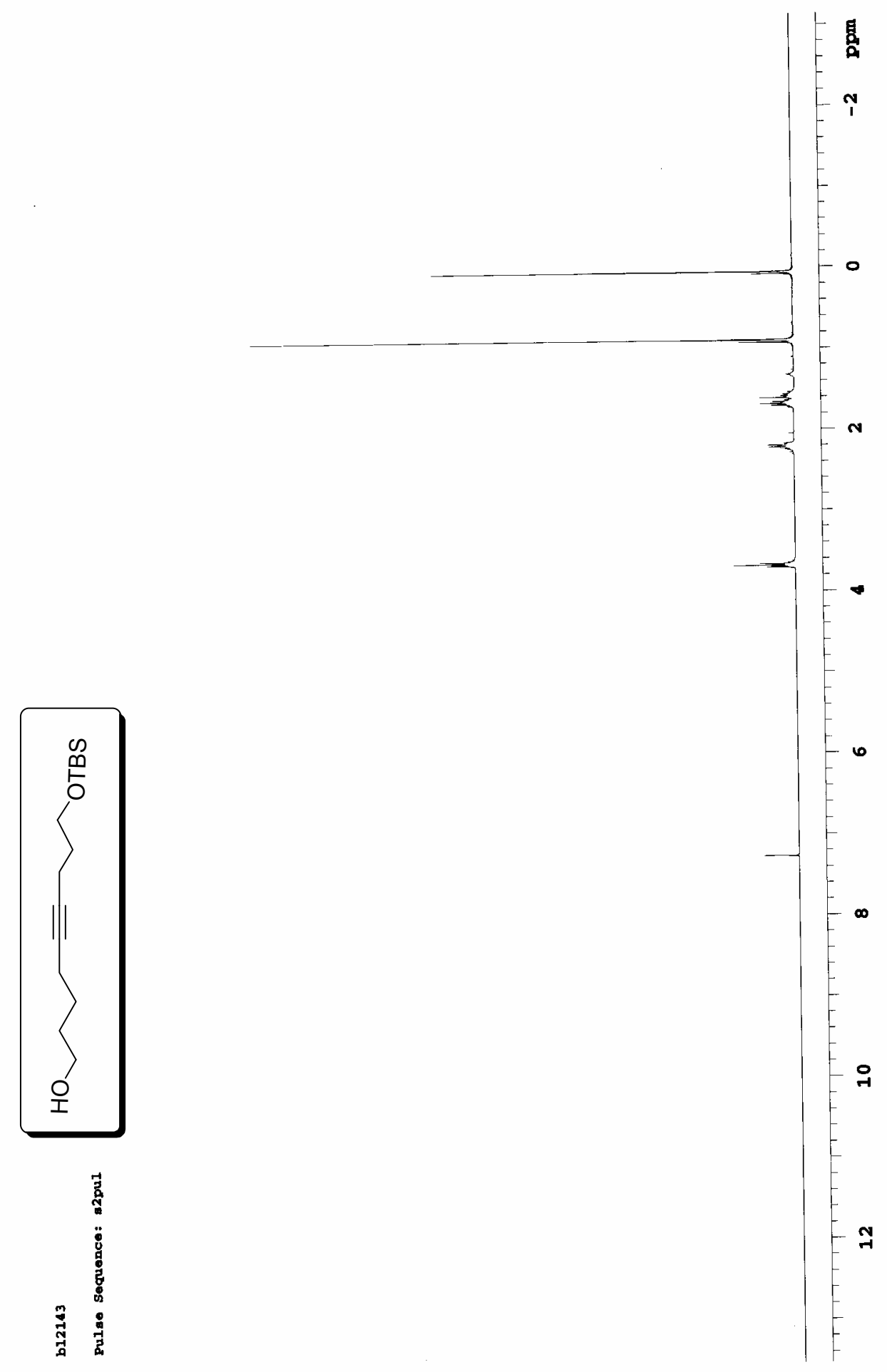




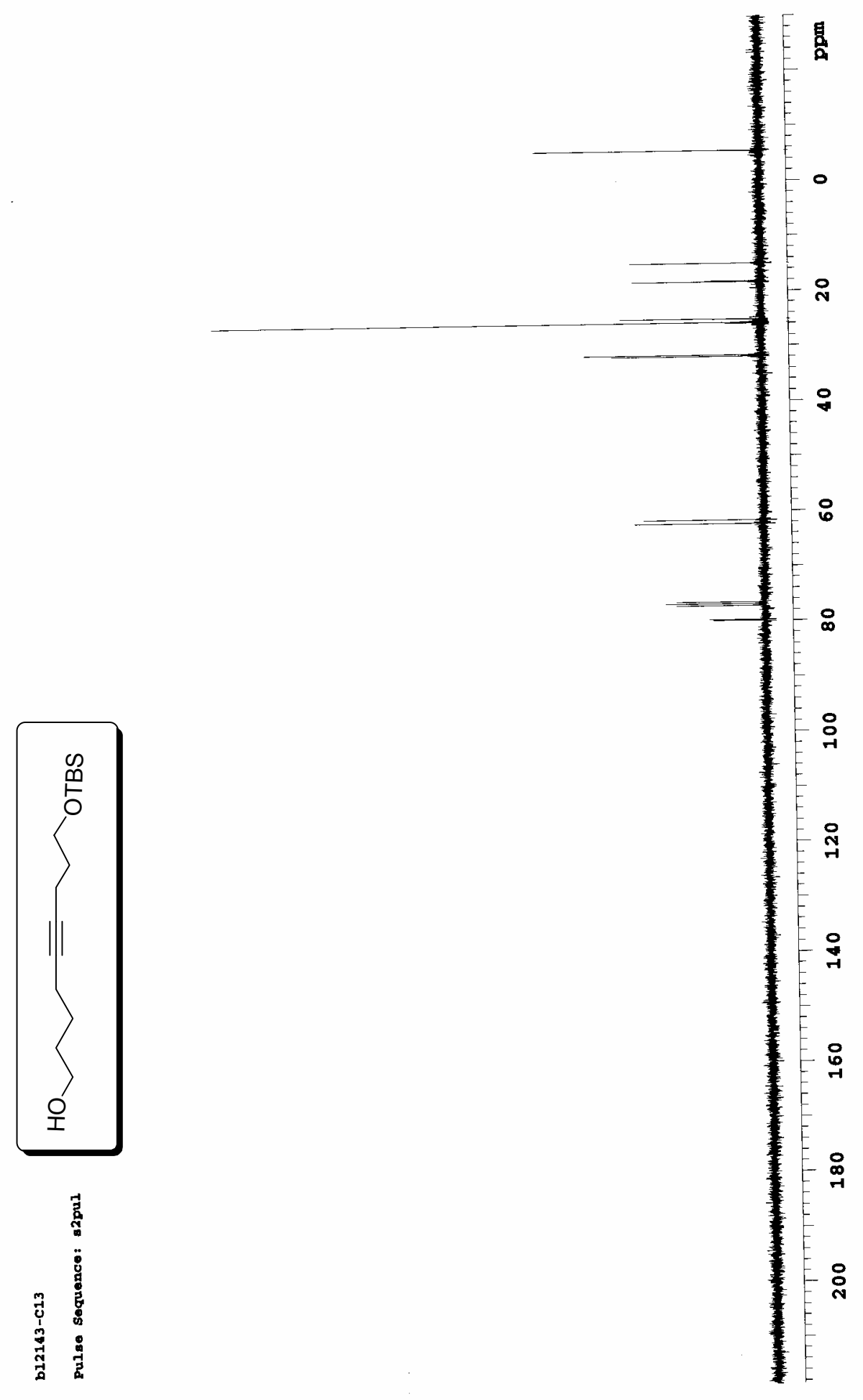




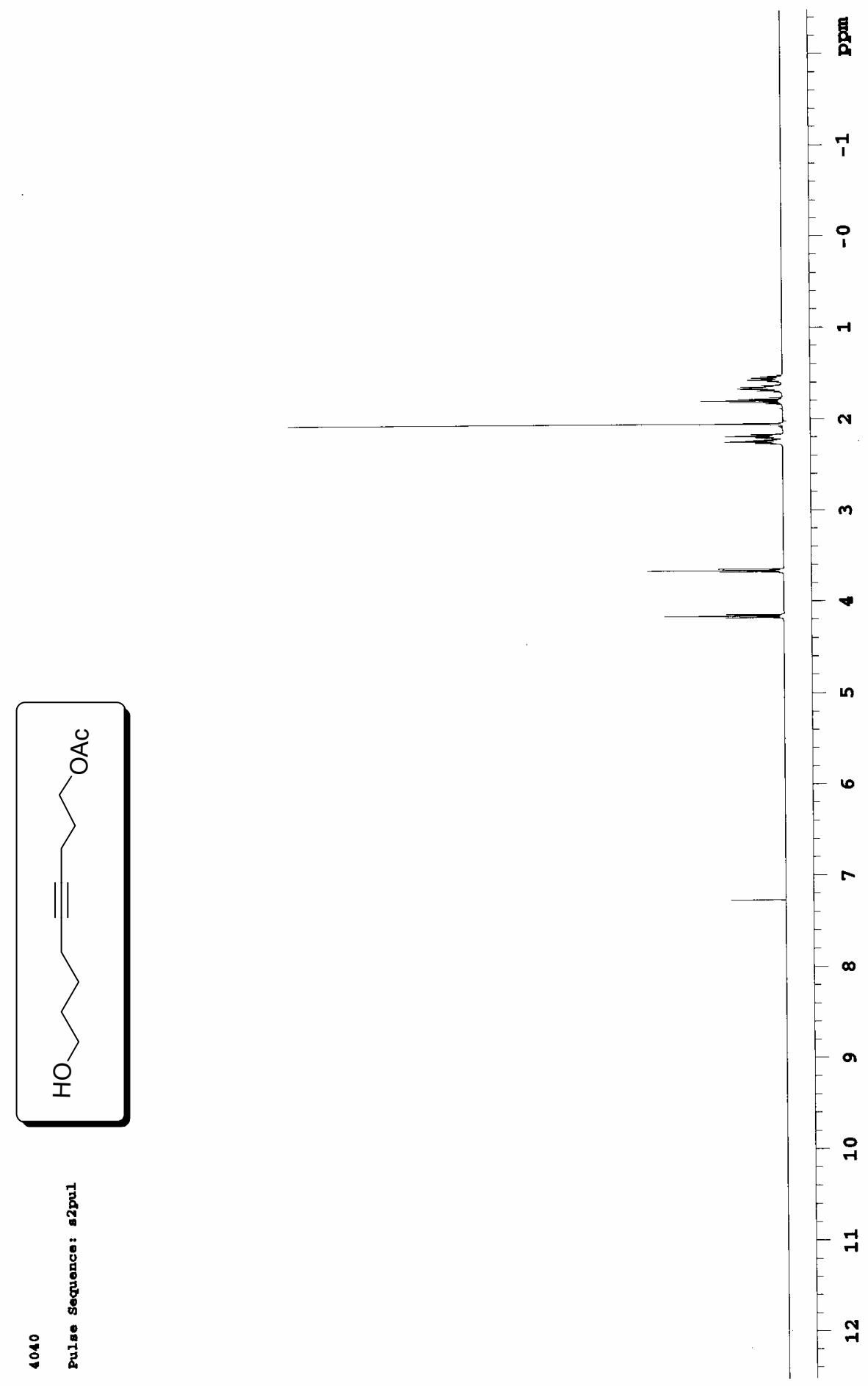




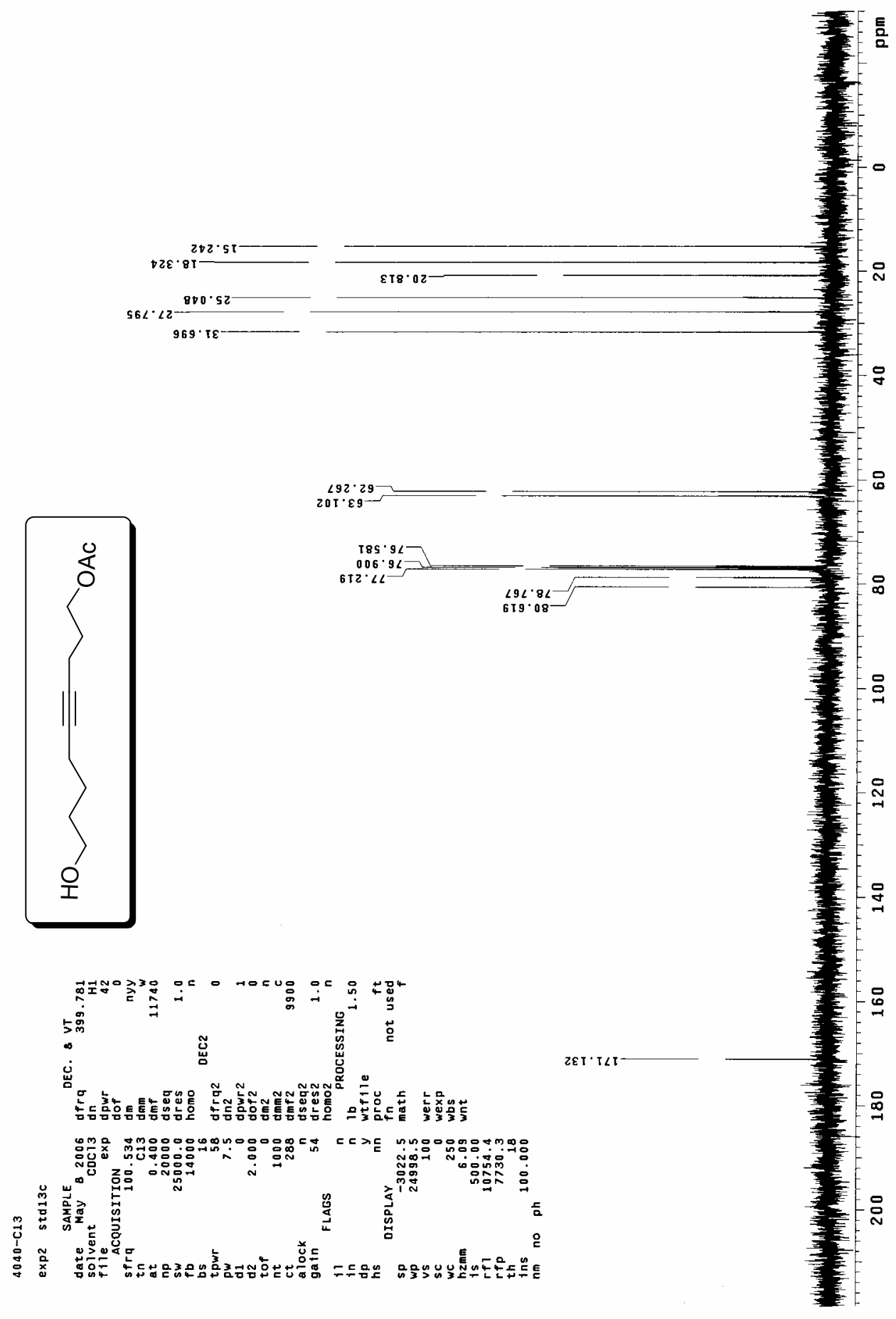




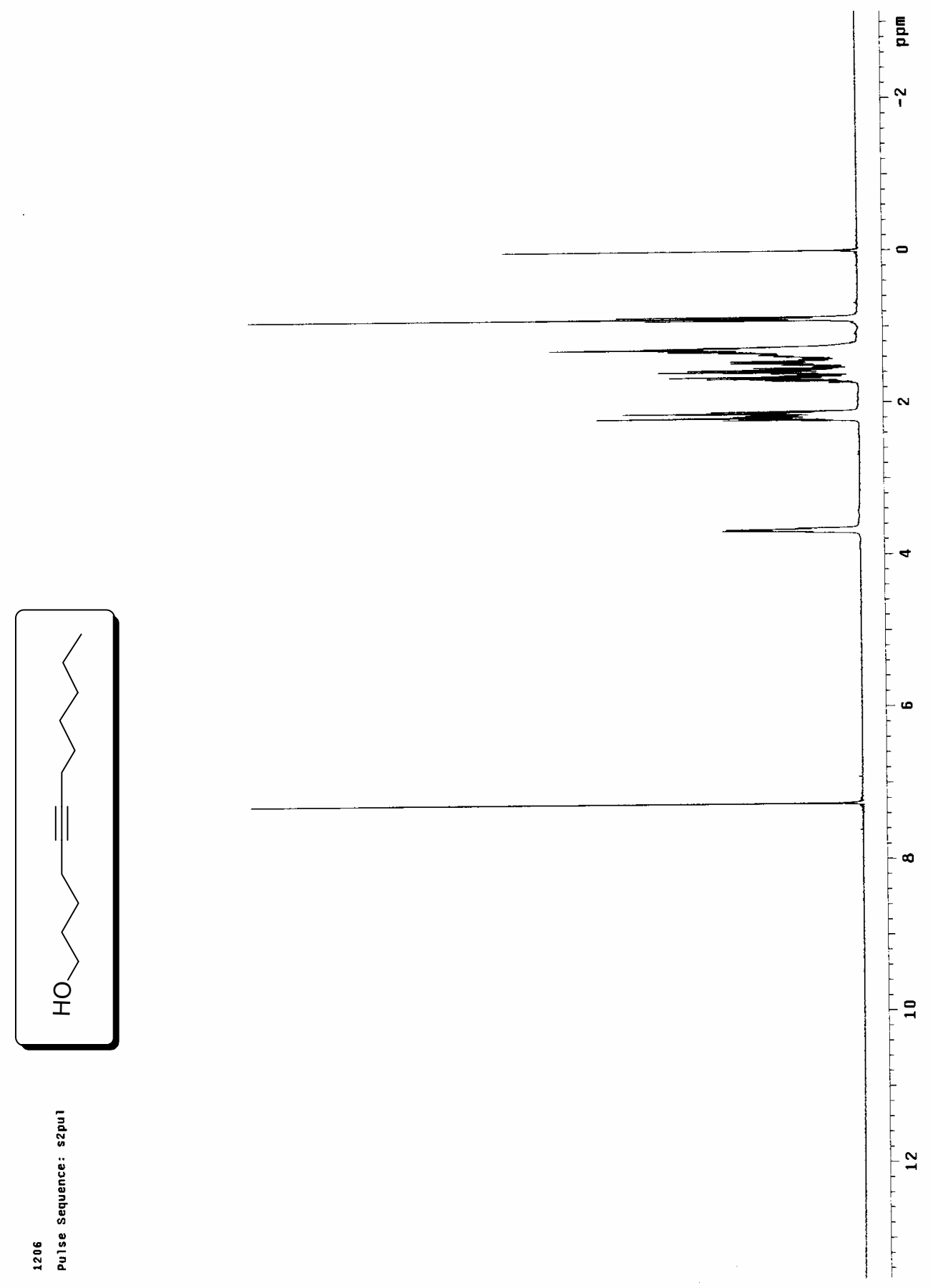

$-21-$ 


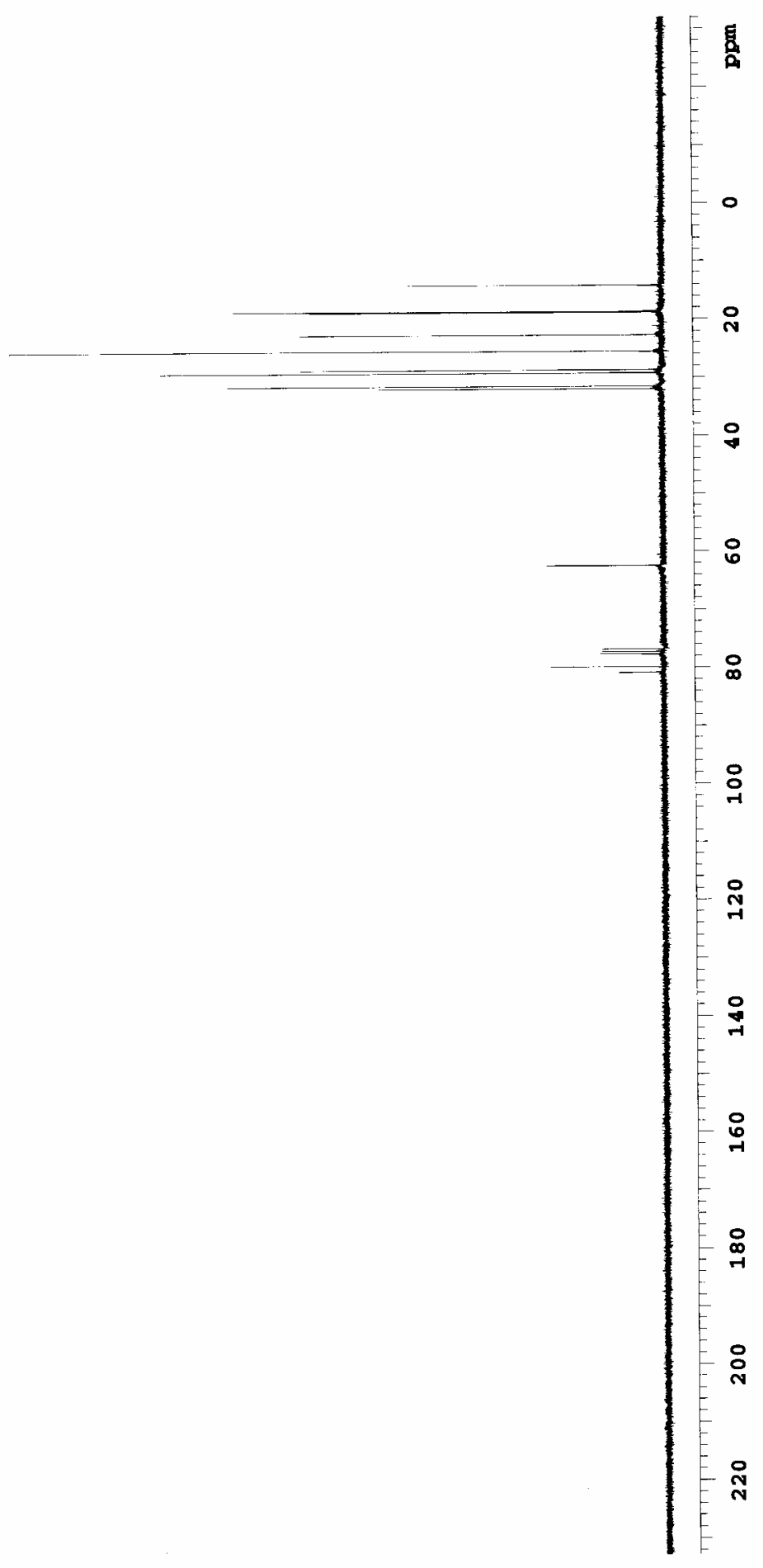

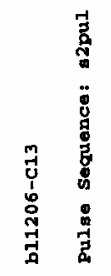




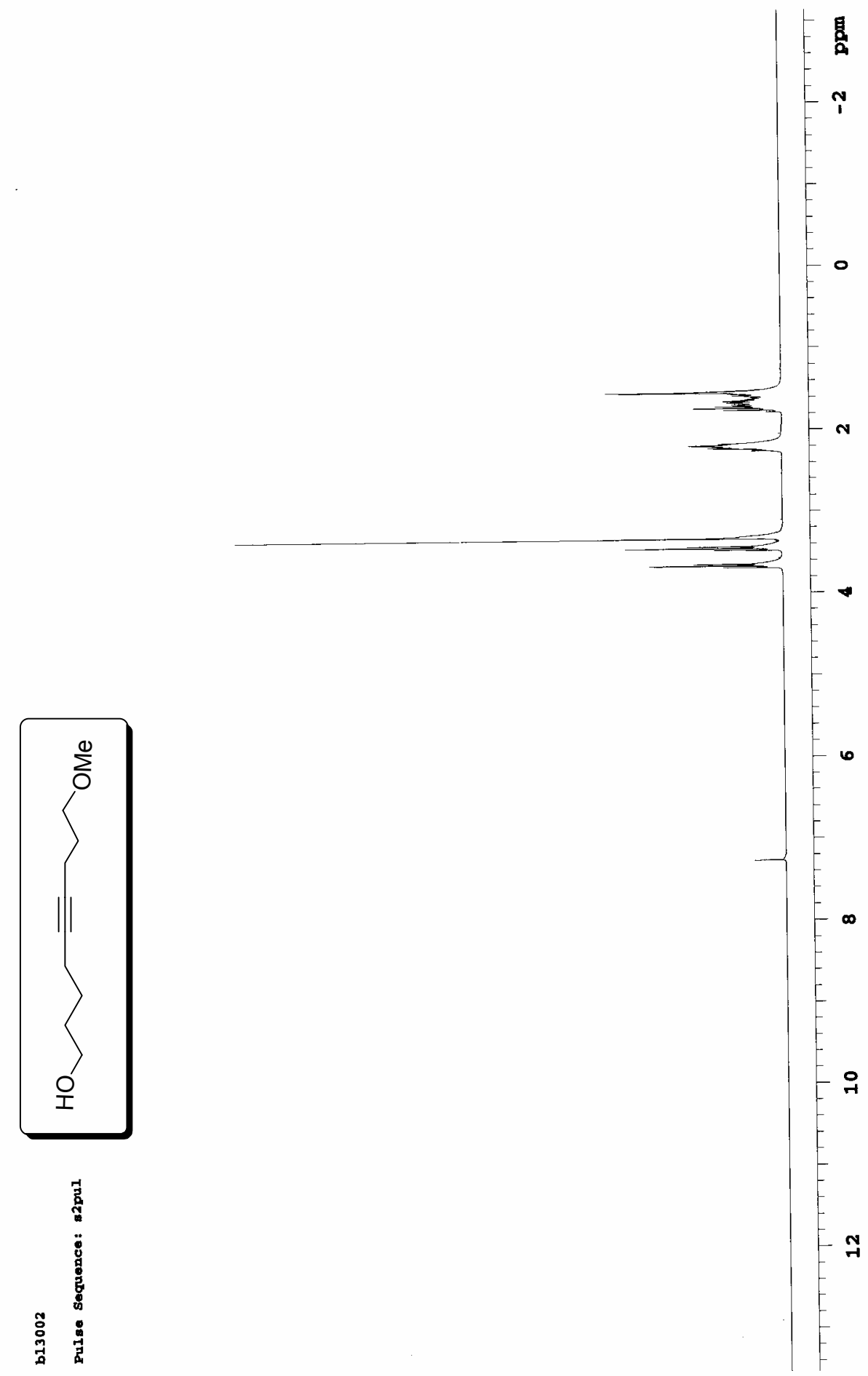




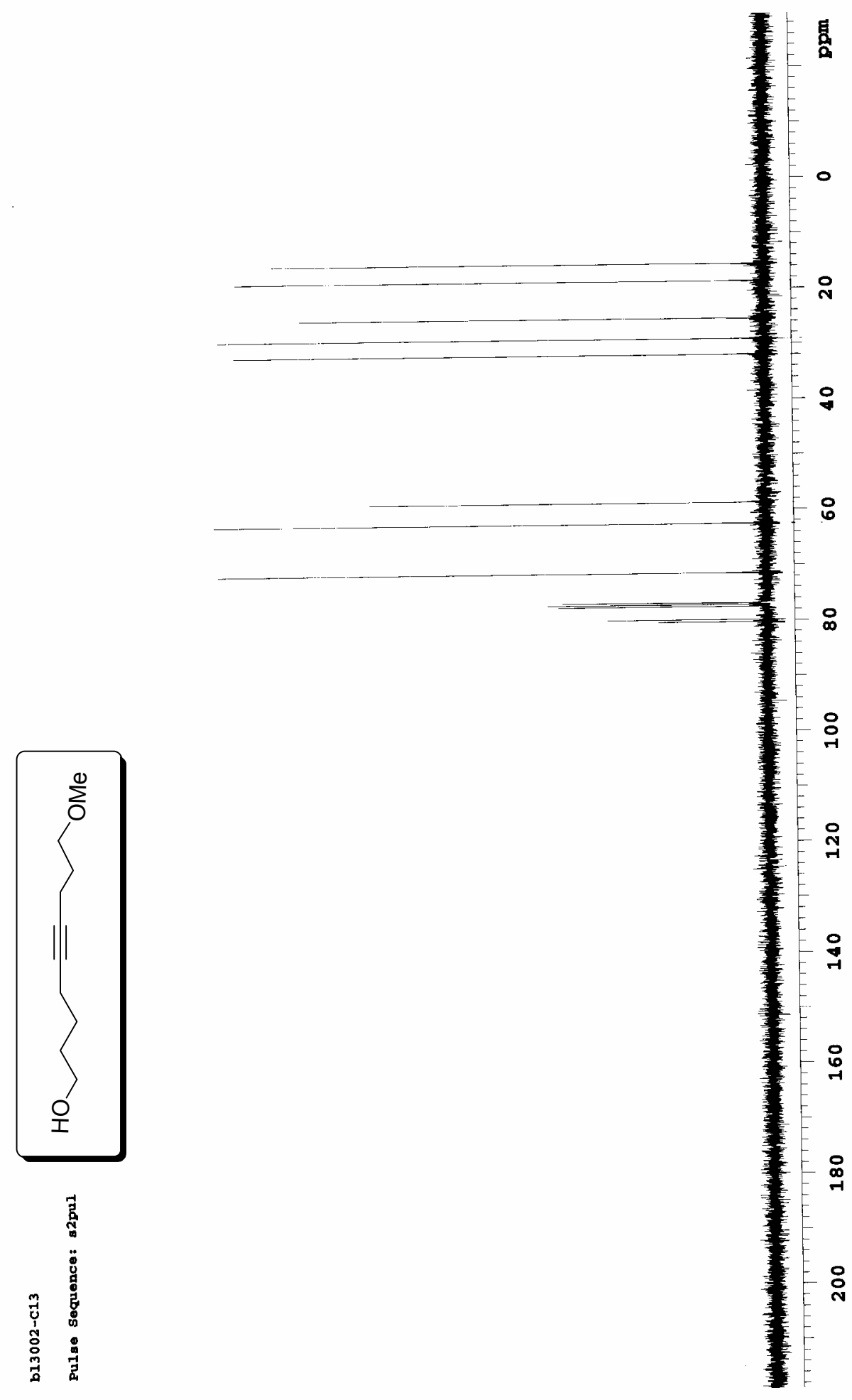




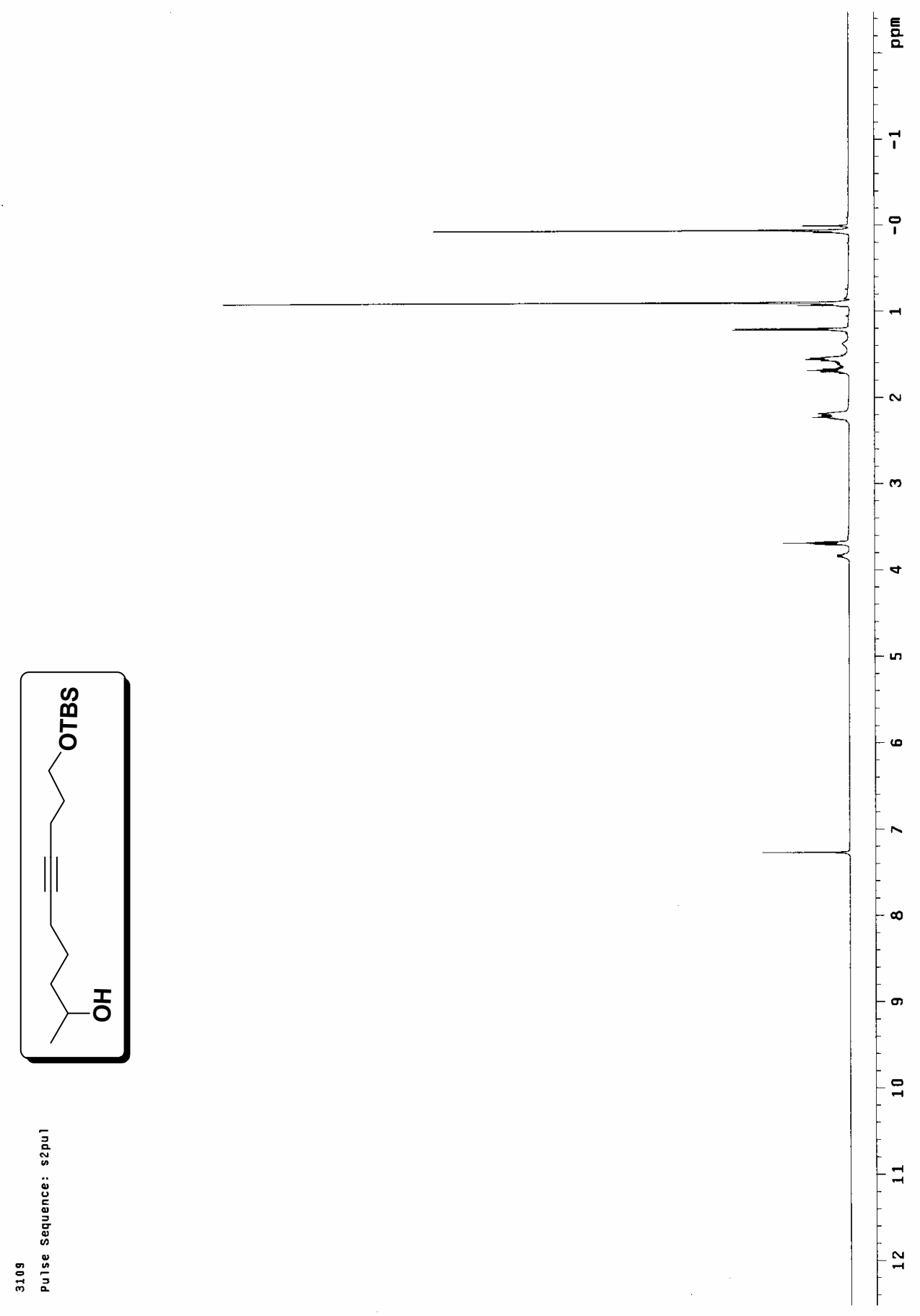



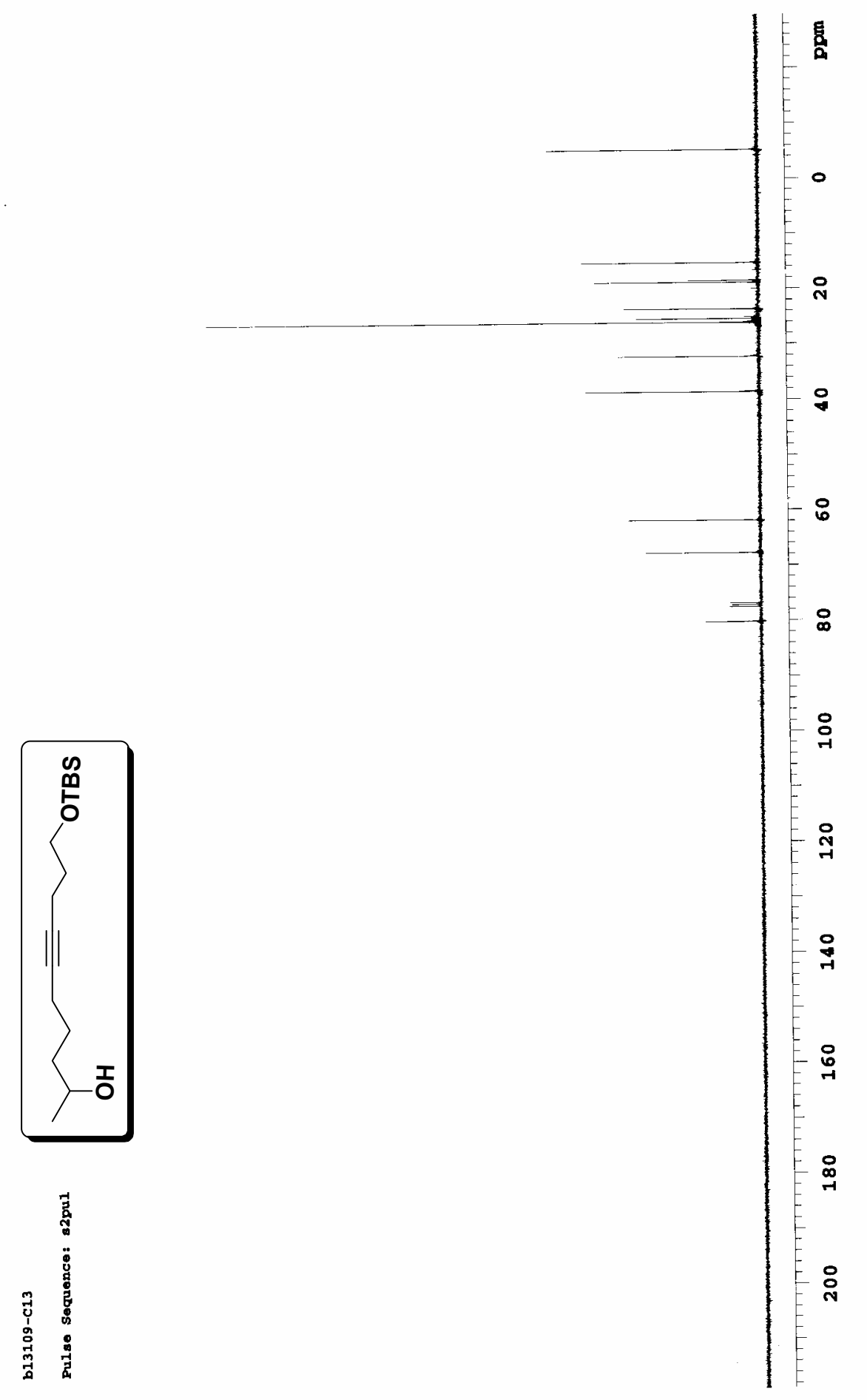


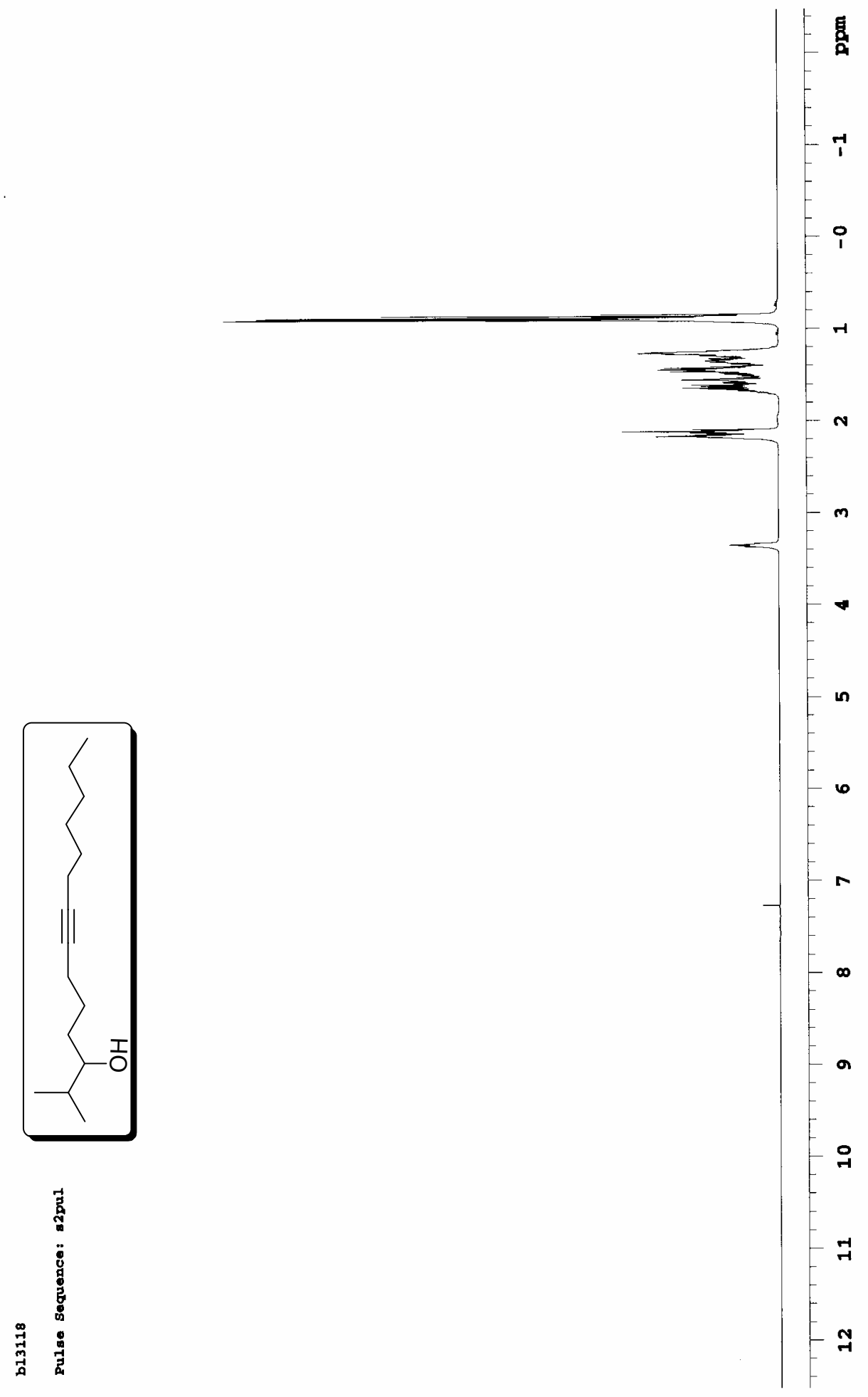



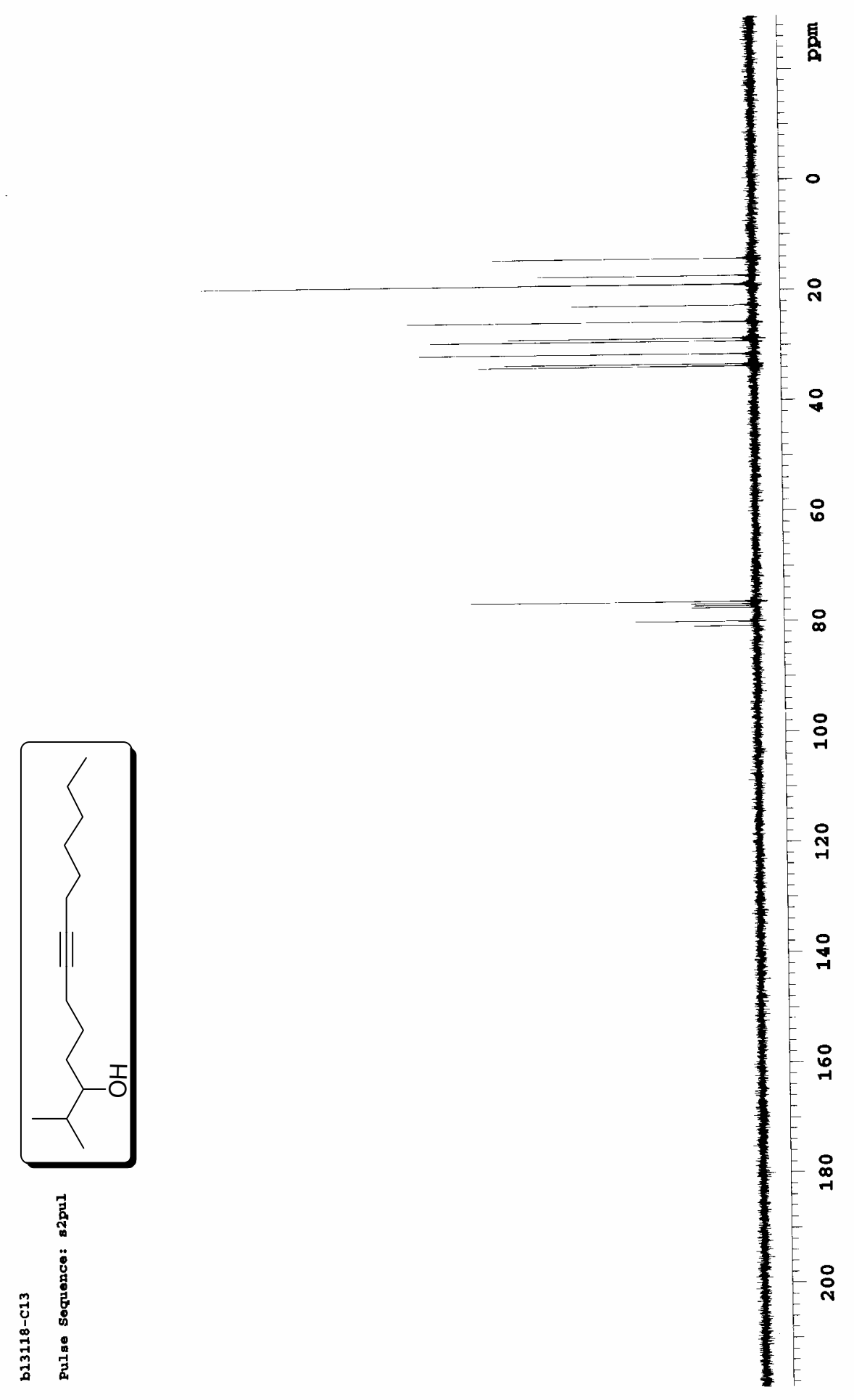


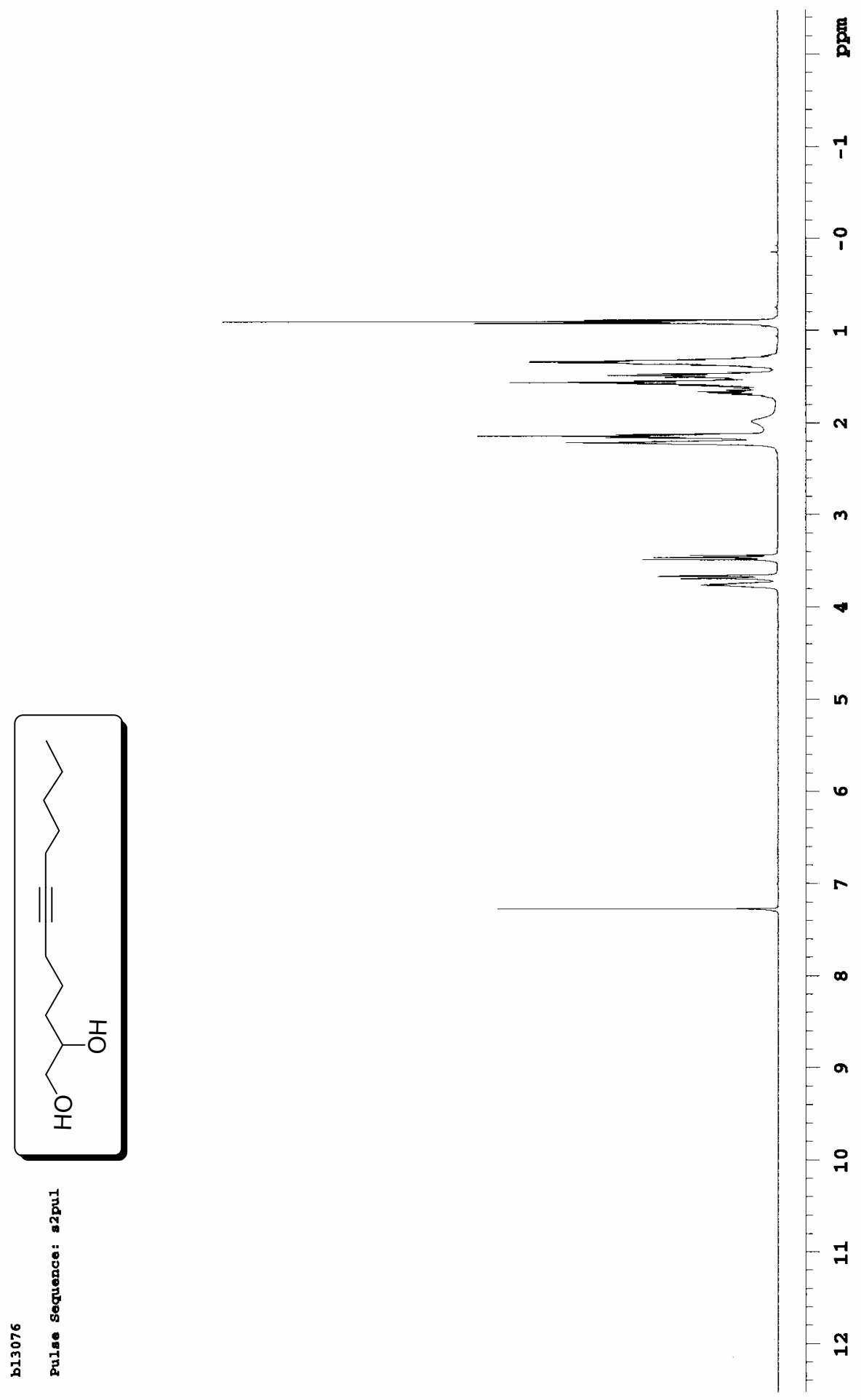



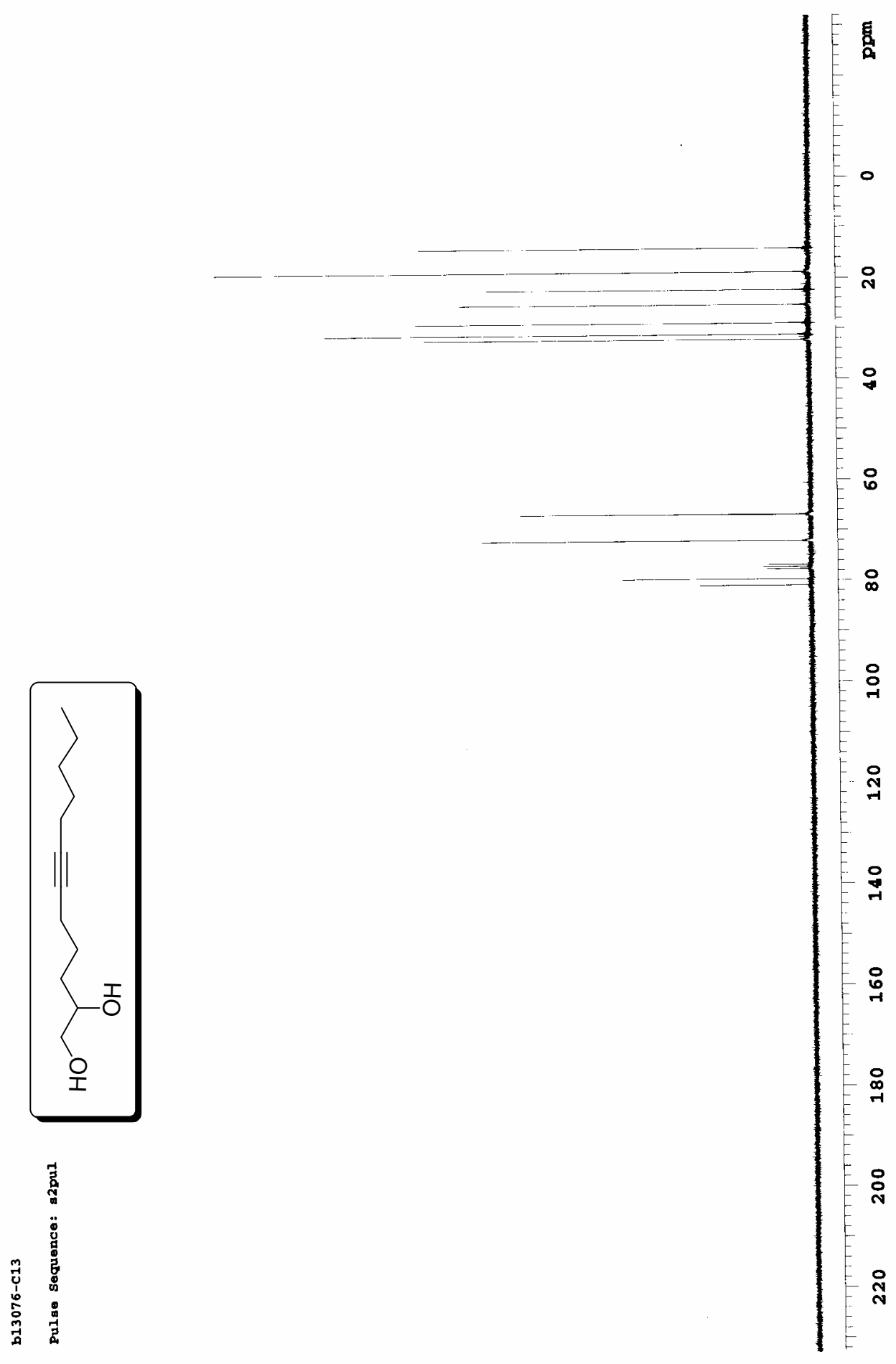


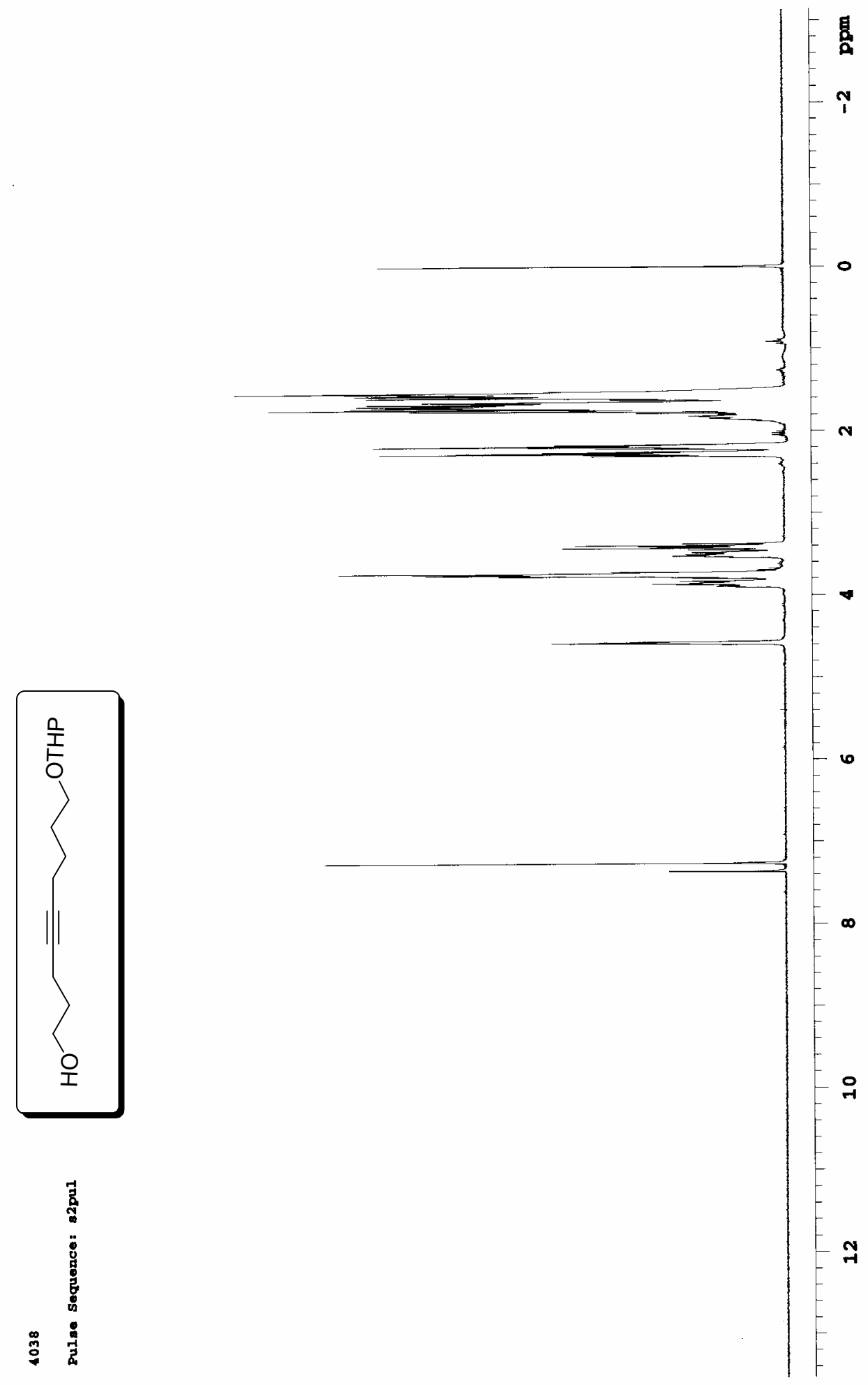

$-31-$ 

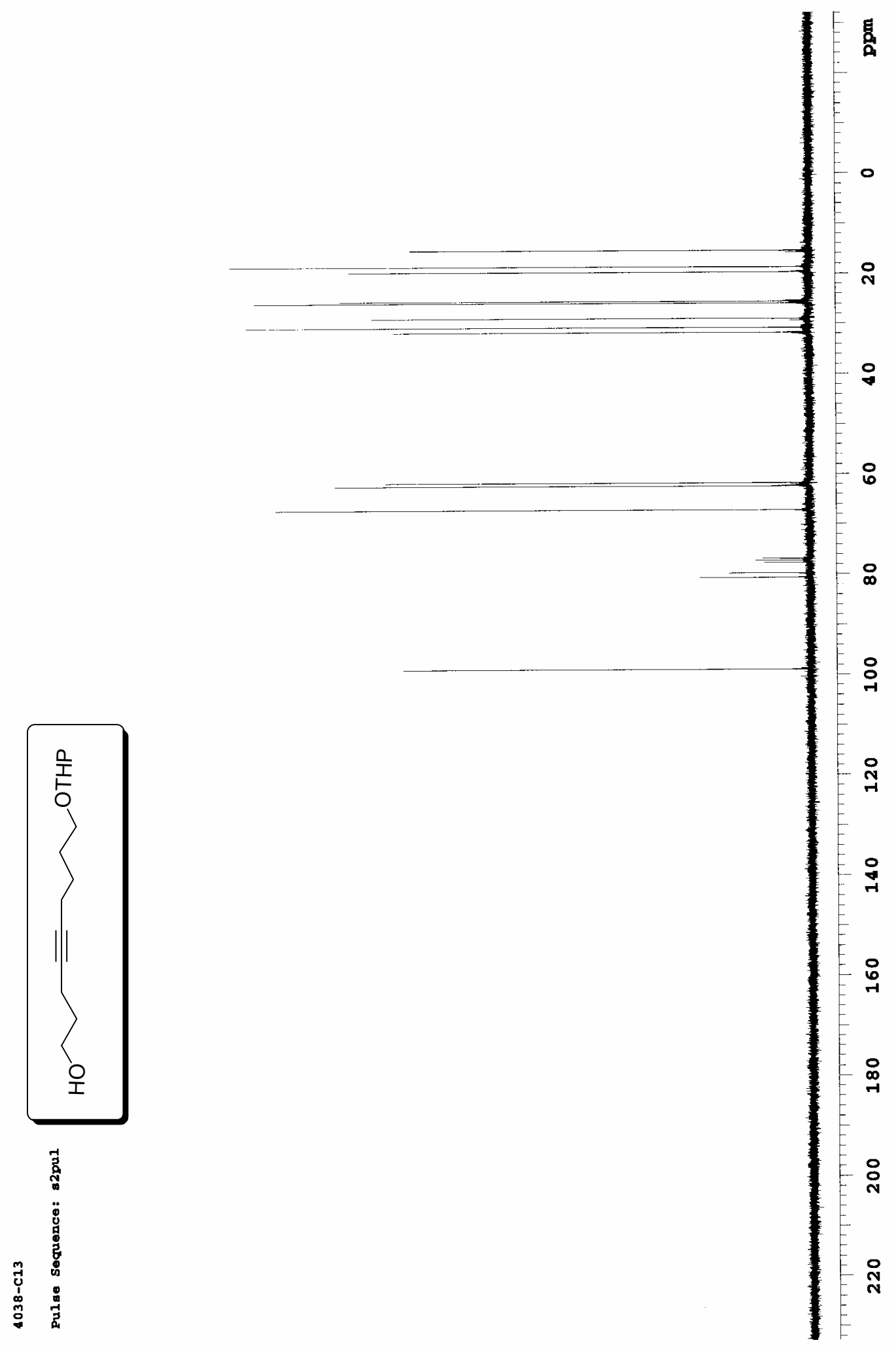


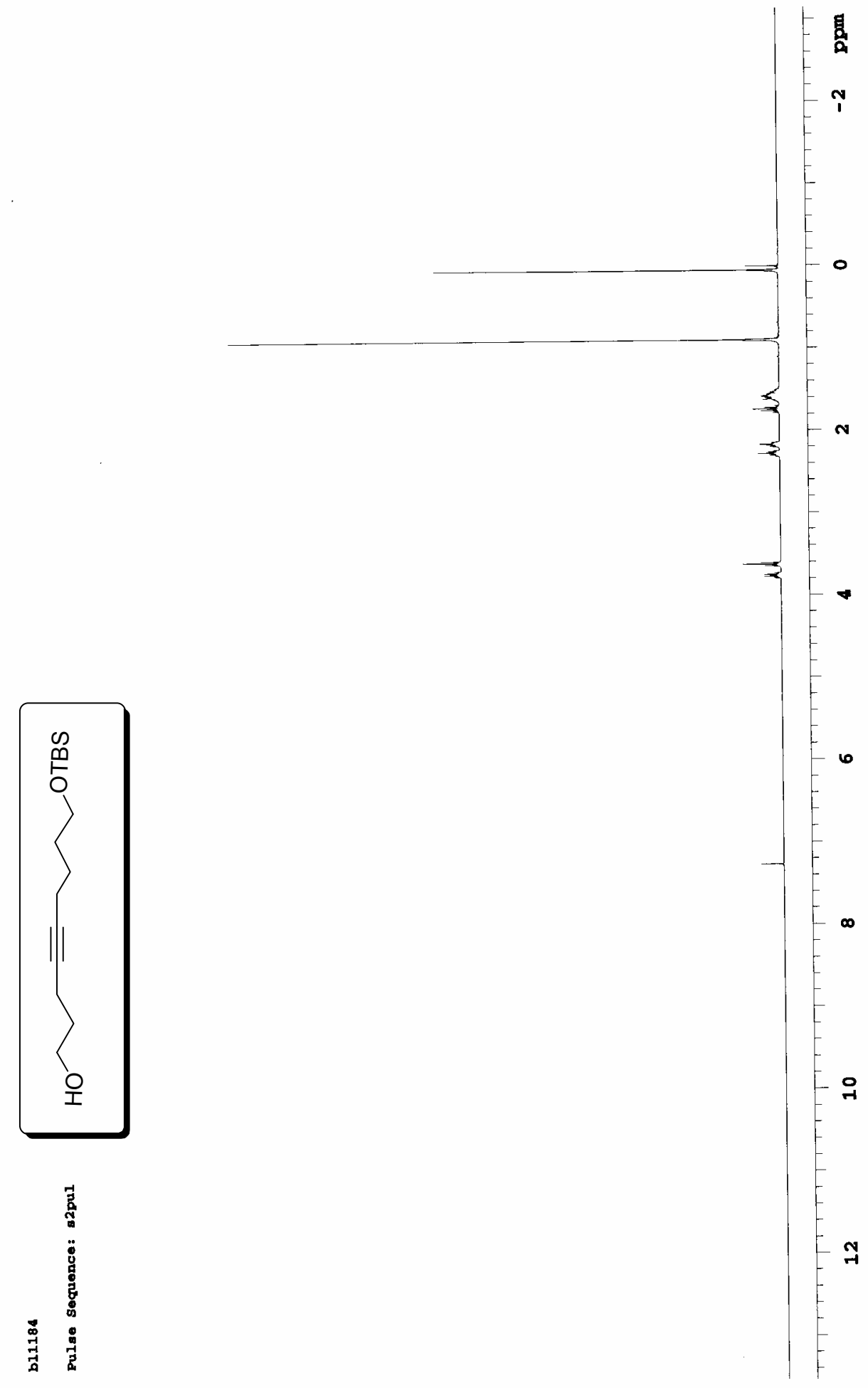




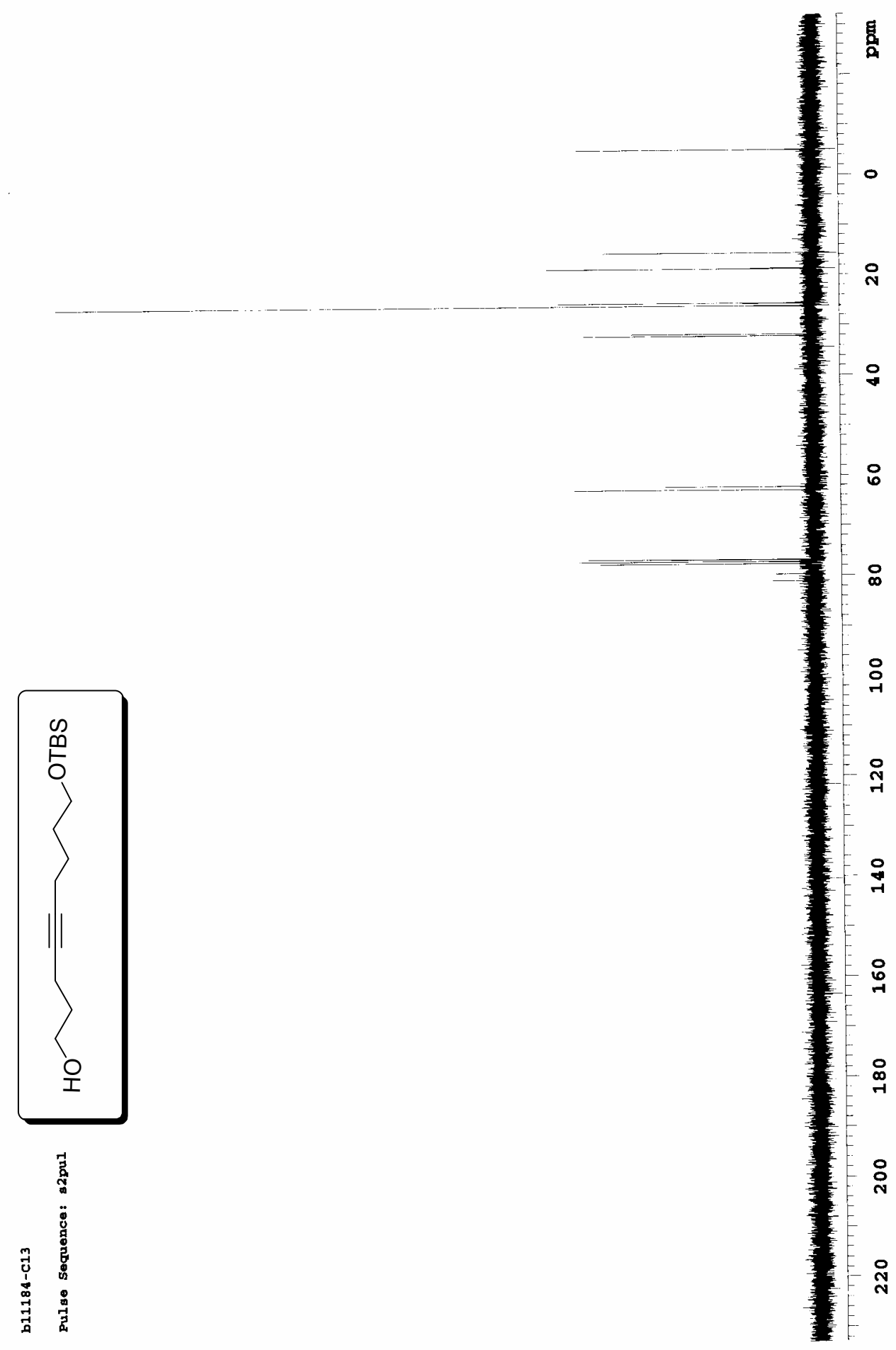




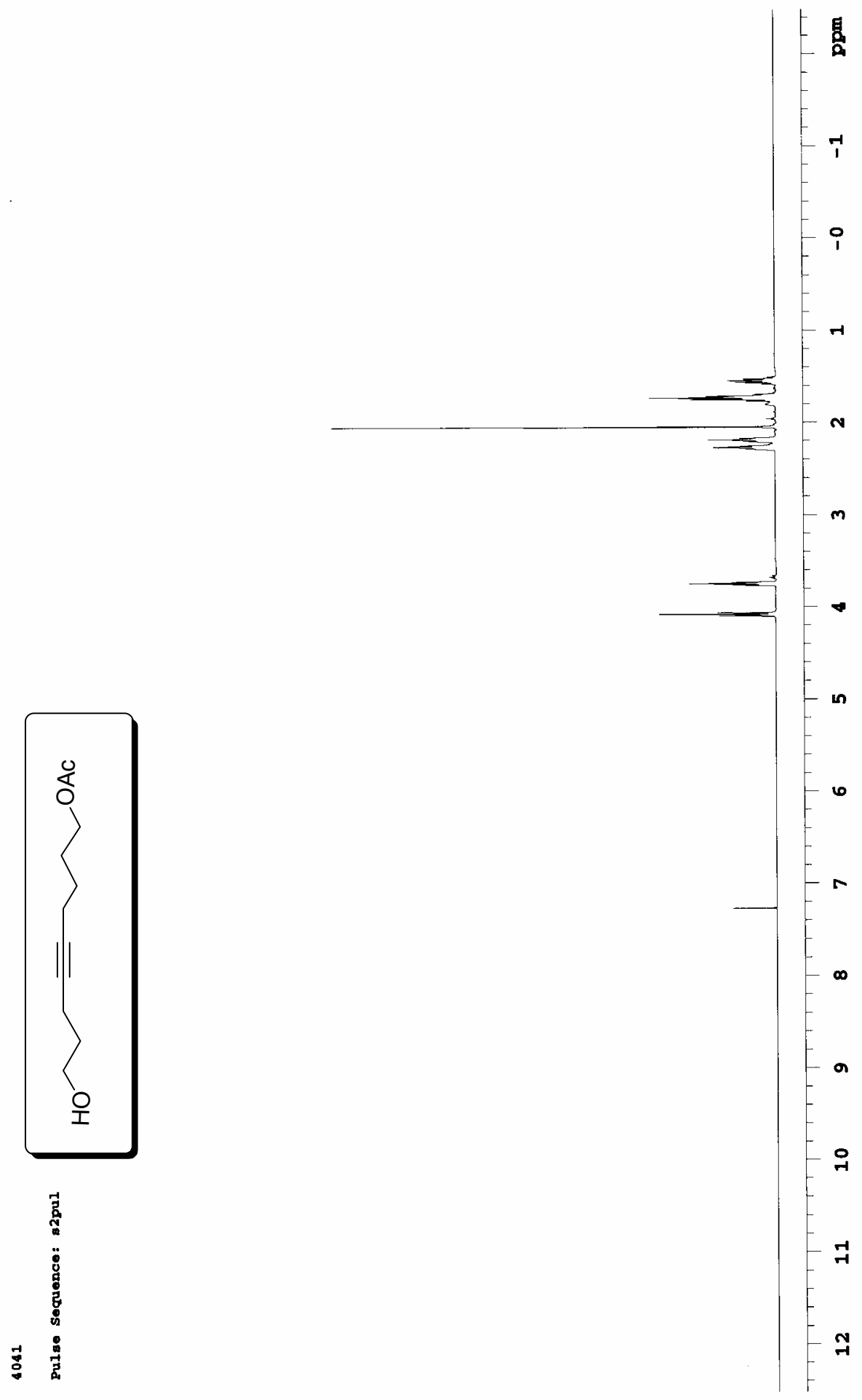




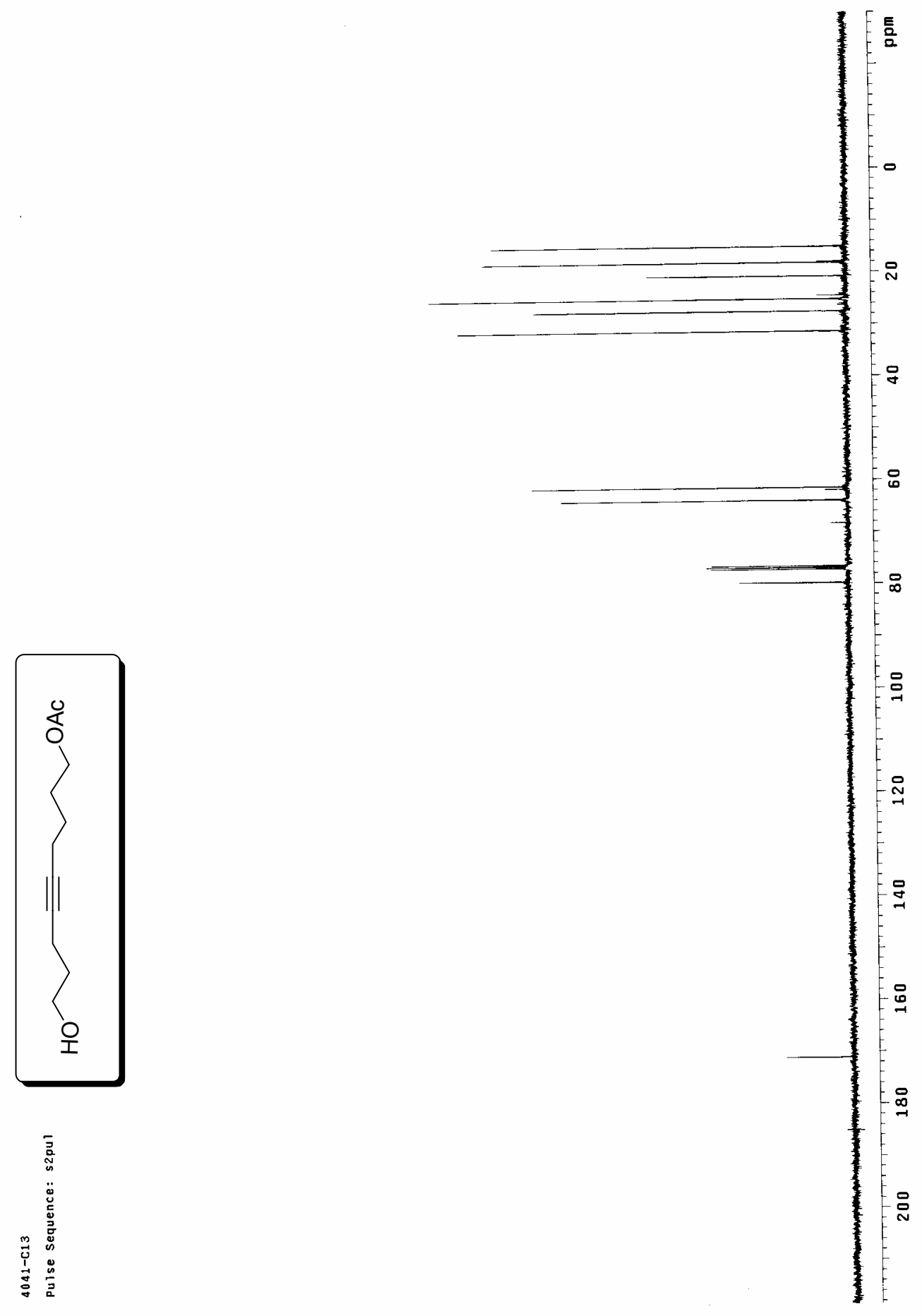




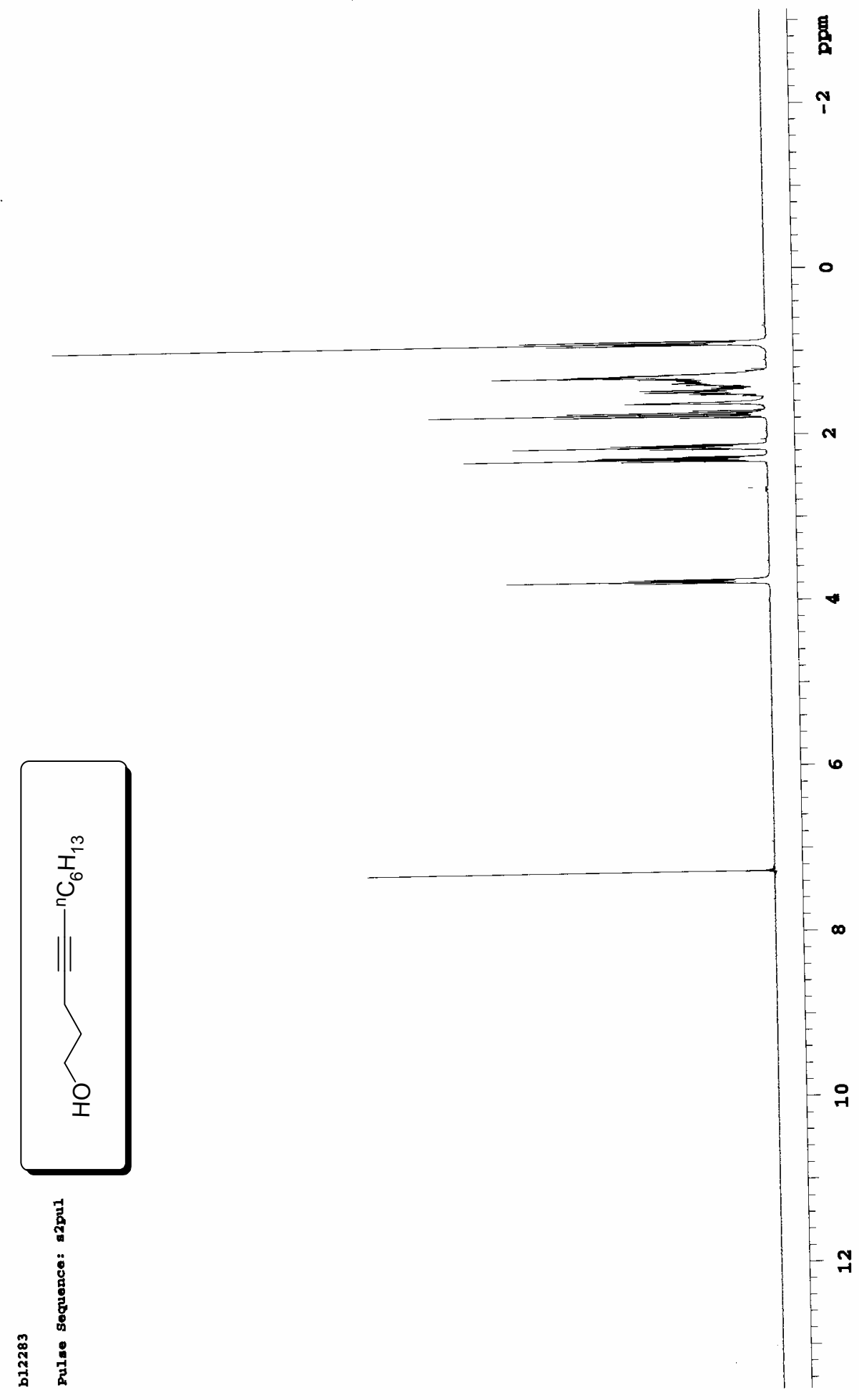



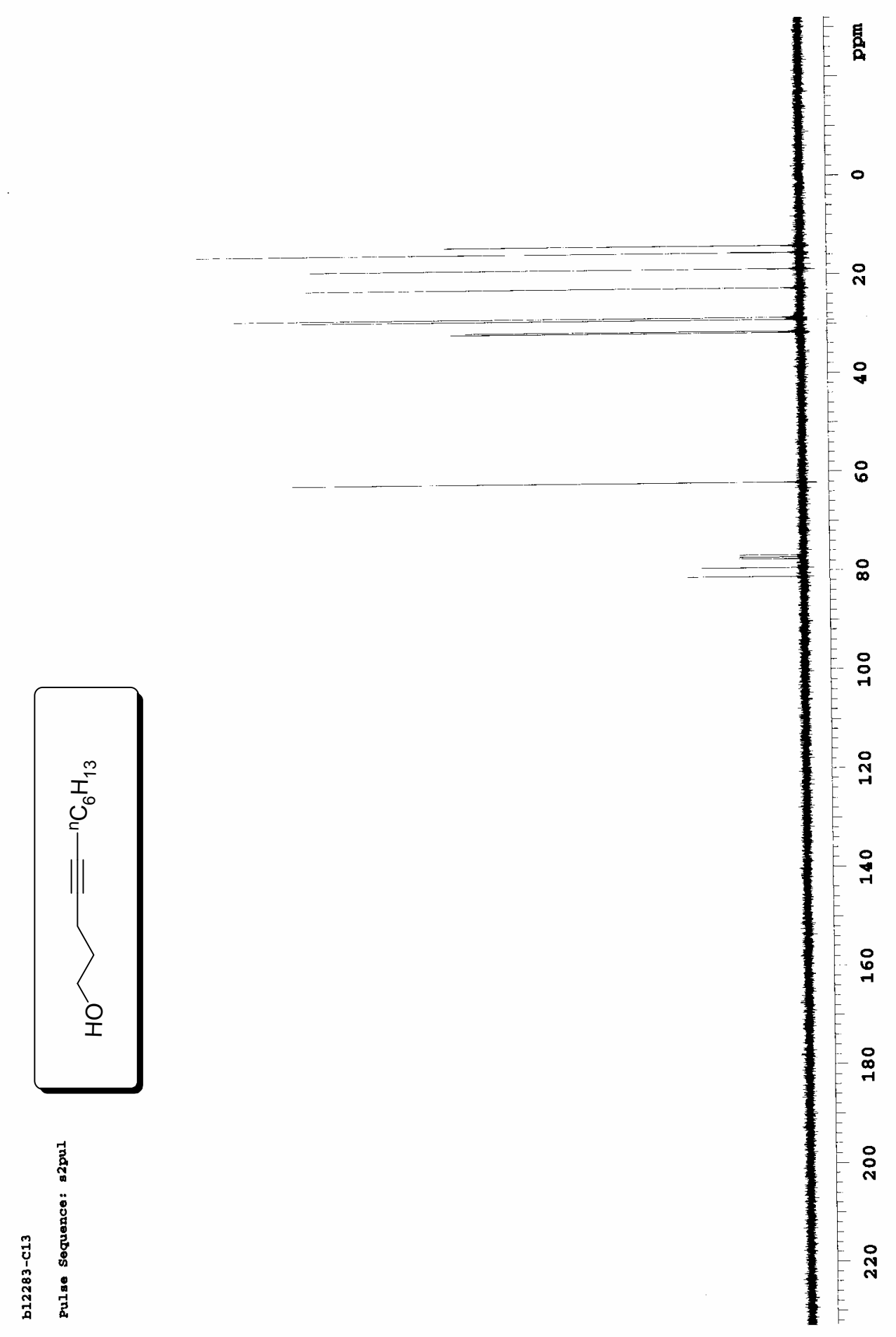


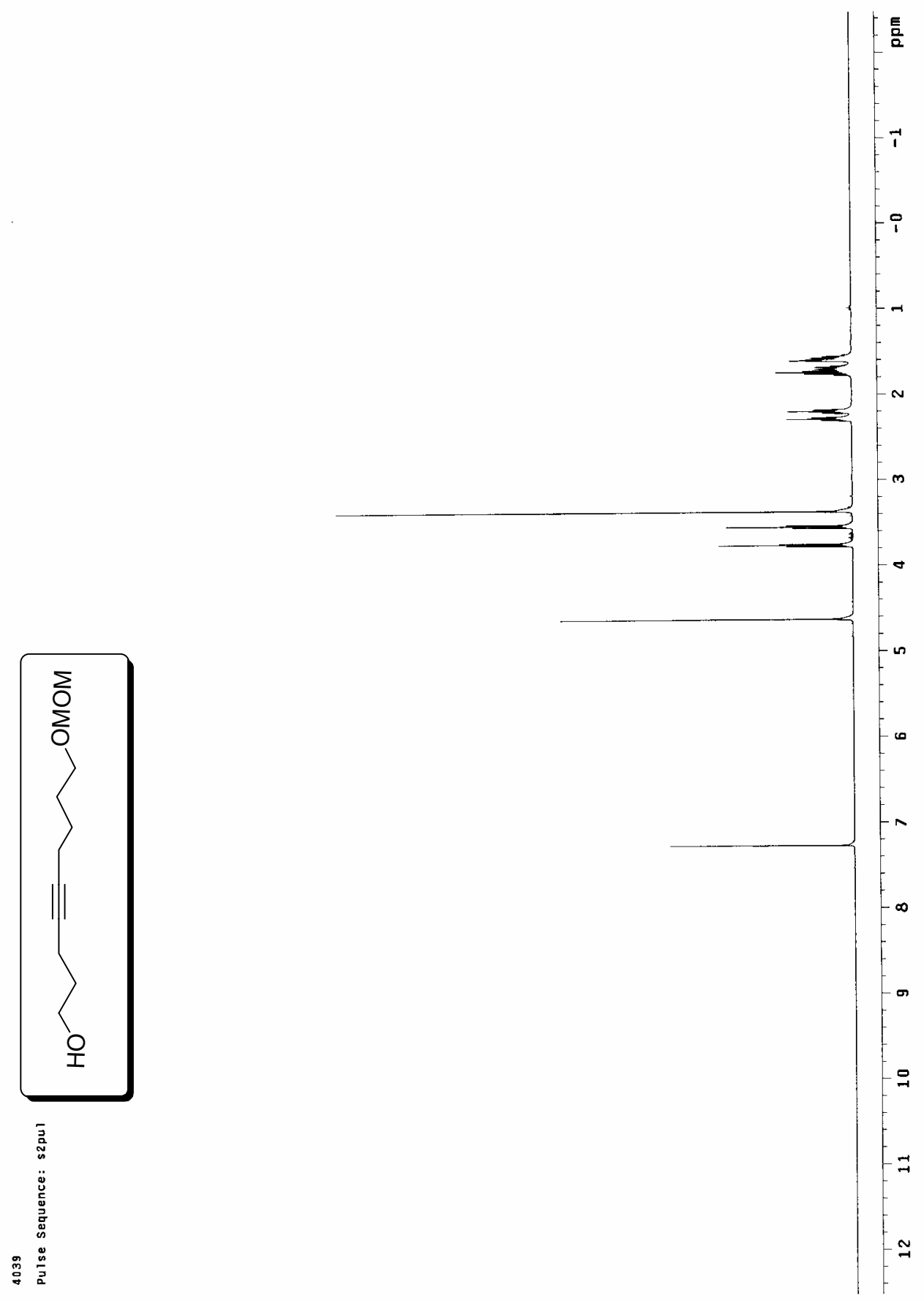




$$
\text { if }
$$




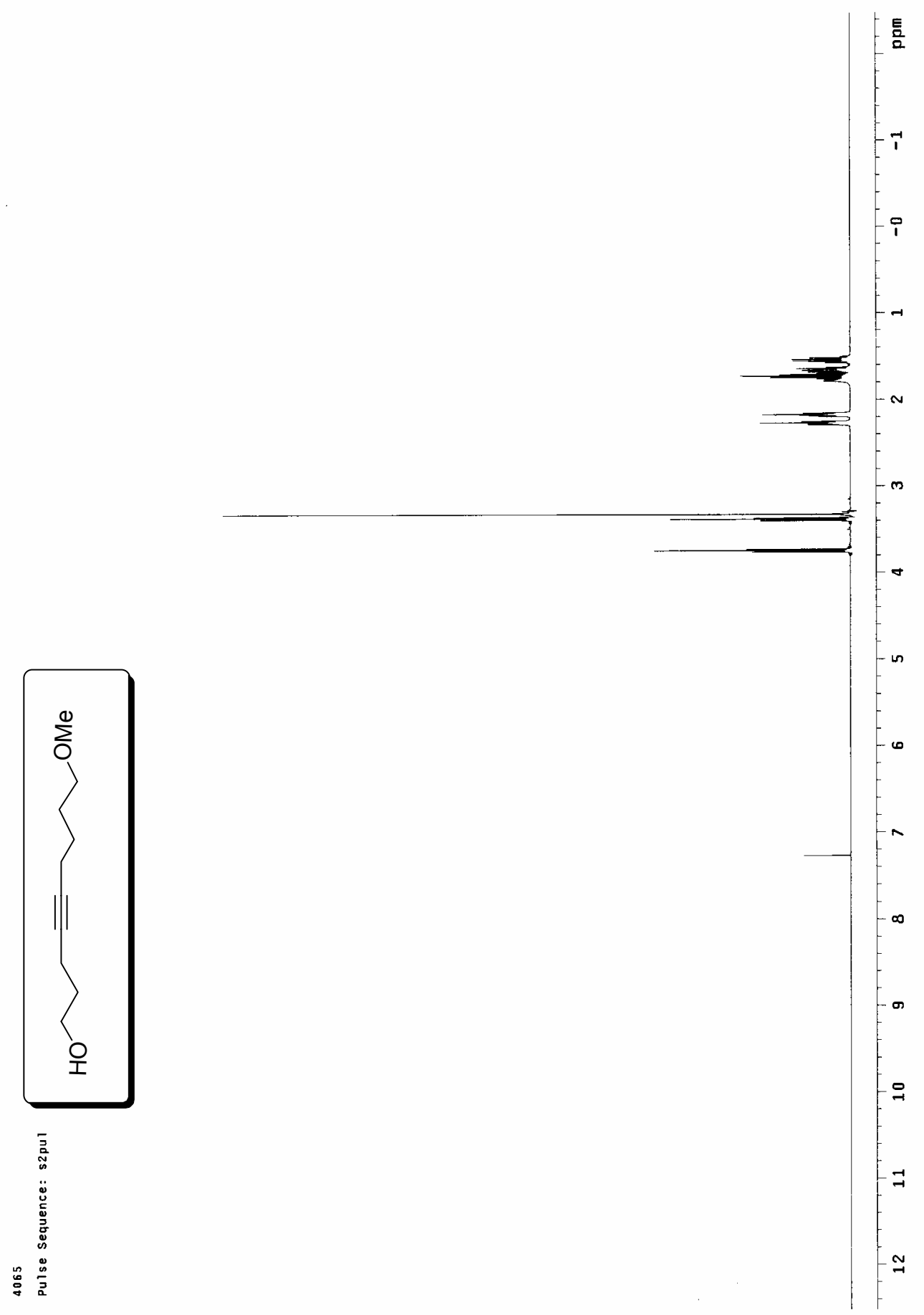



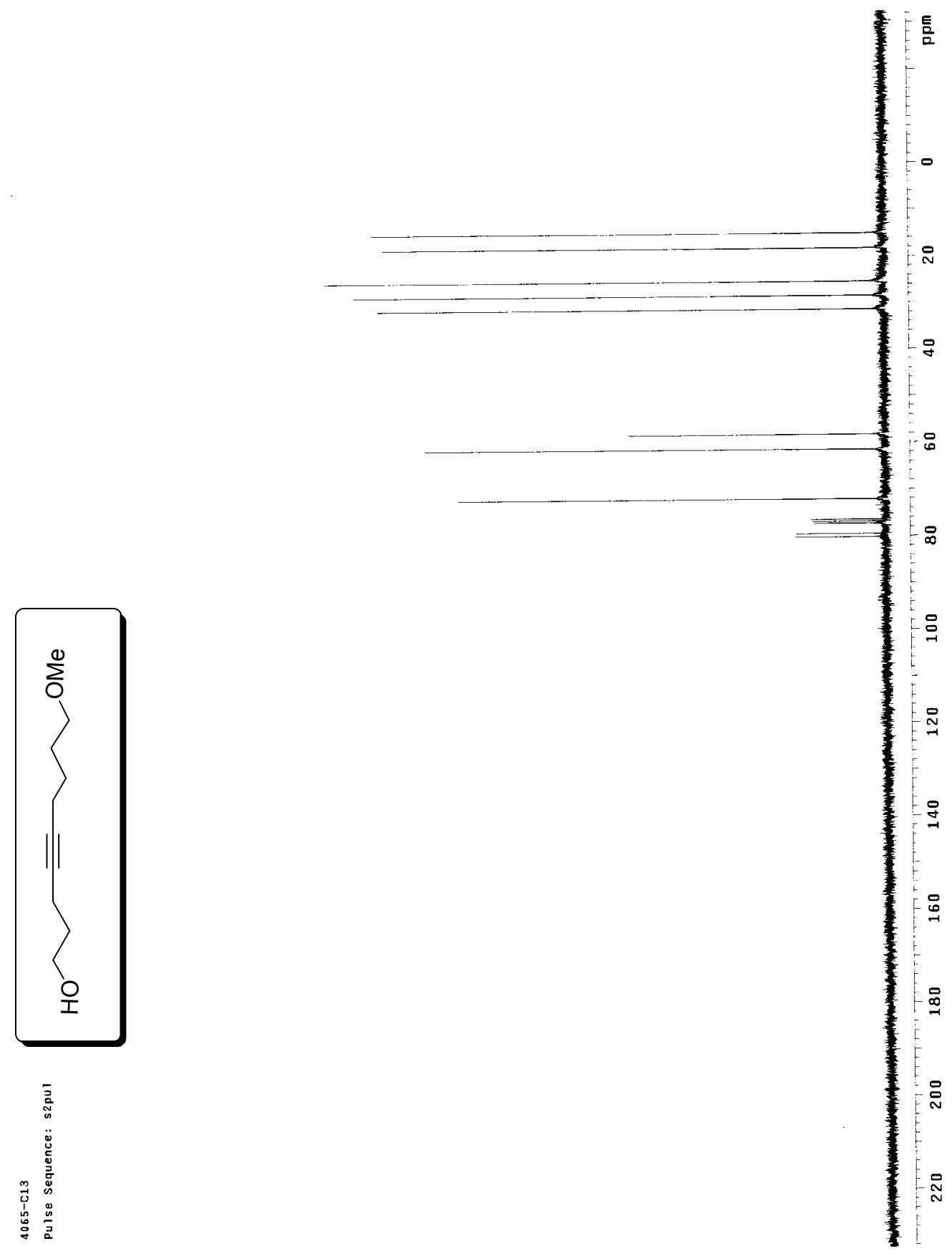
${ }^{1}$ H NMR-spectrum for Table 2, entry 3 (400 MHz)

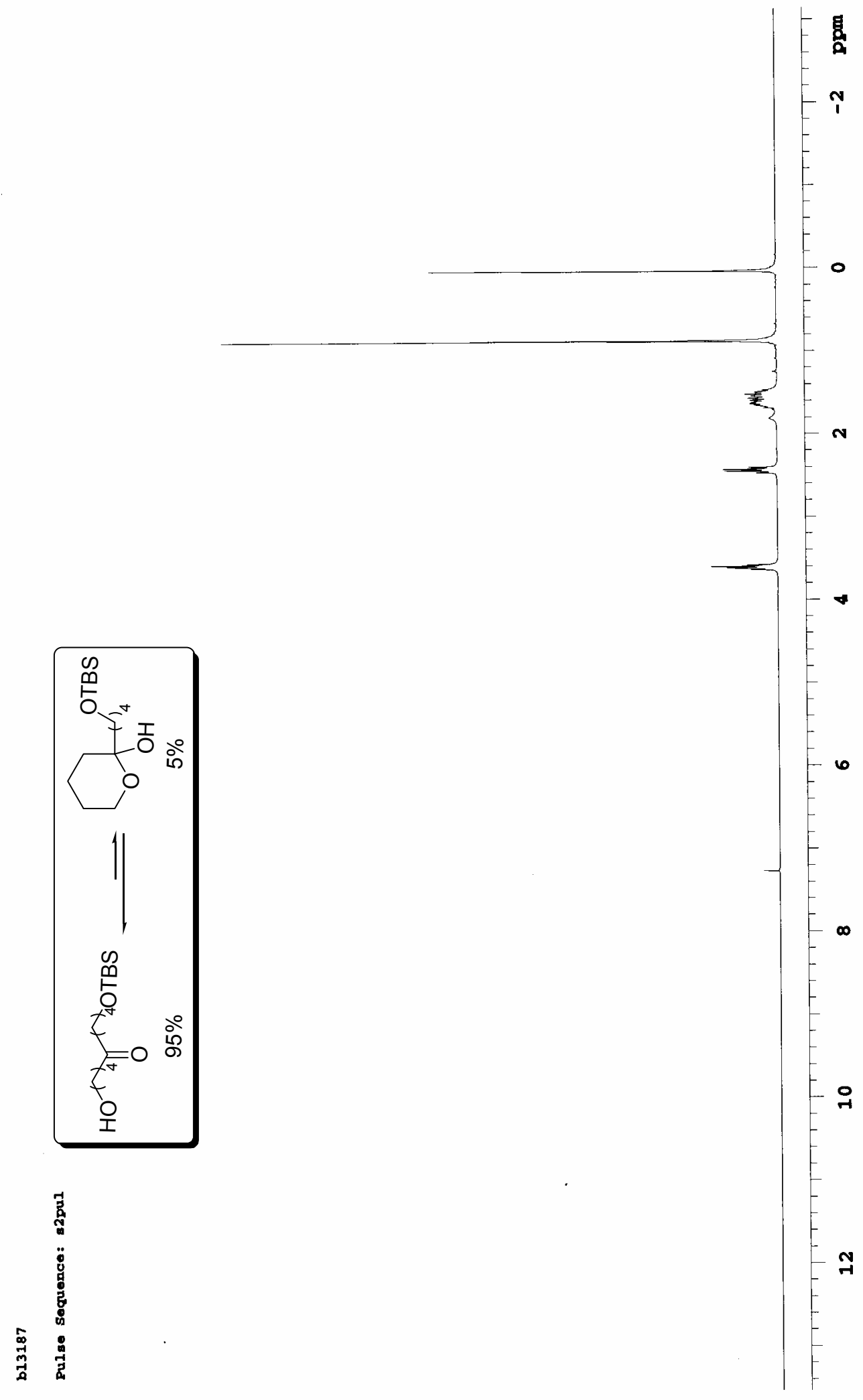


${ }^{13} \mathrm{C}$ NMR-spectrum for Table 2, entry 3 (100 MHz)

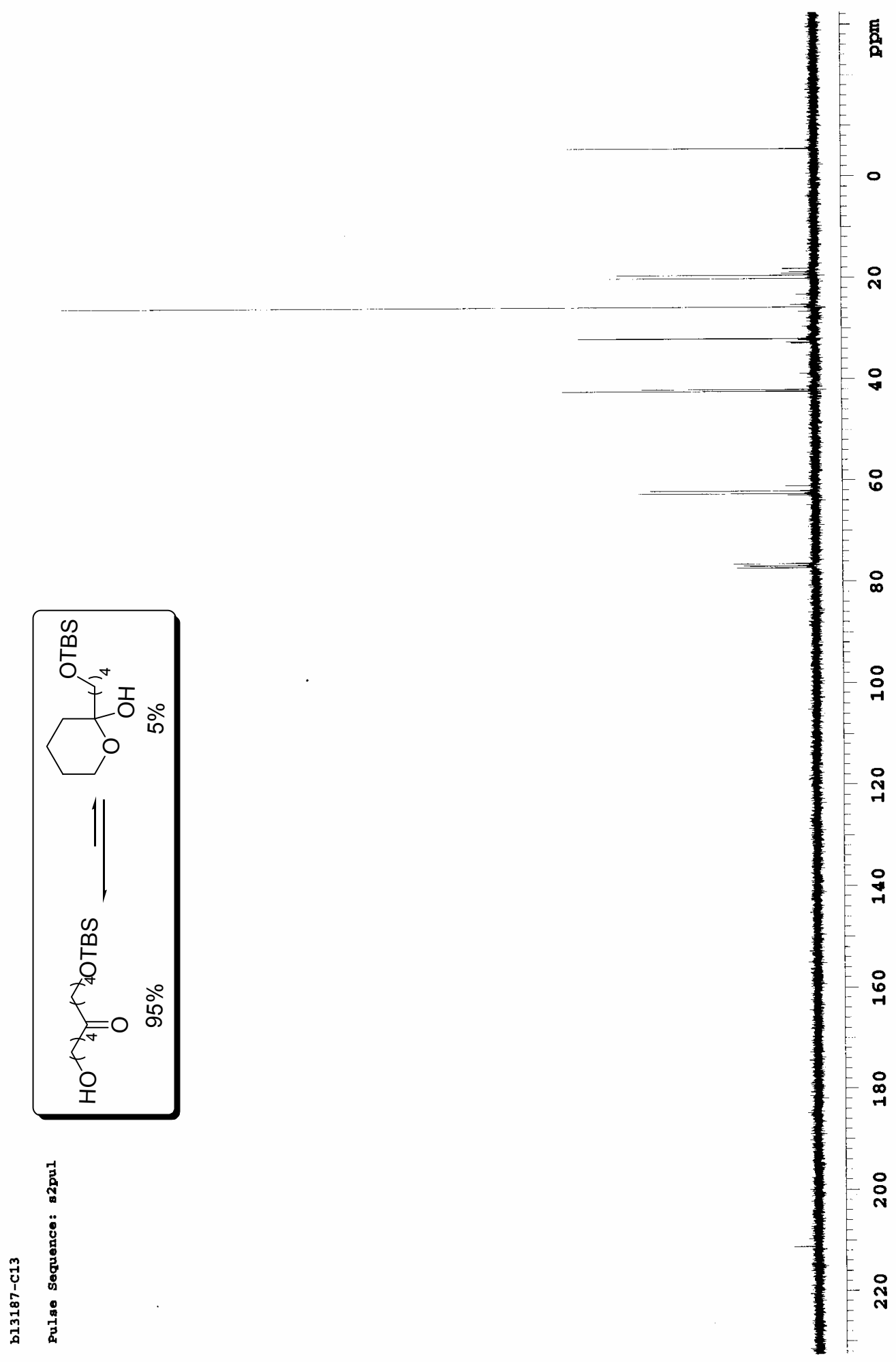


${ }^{1}$ H NMR-spectrum for Table 2, entry 4 (400 MHz)

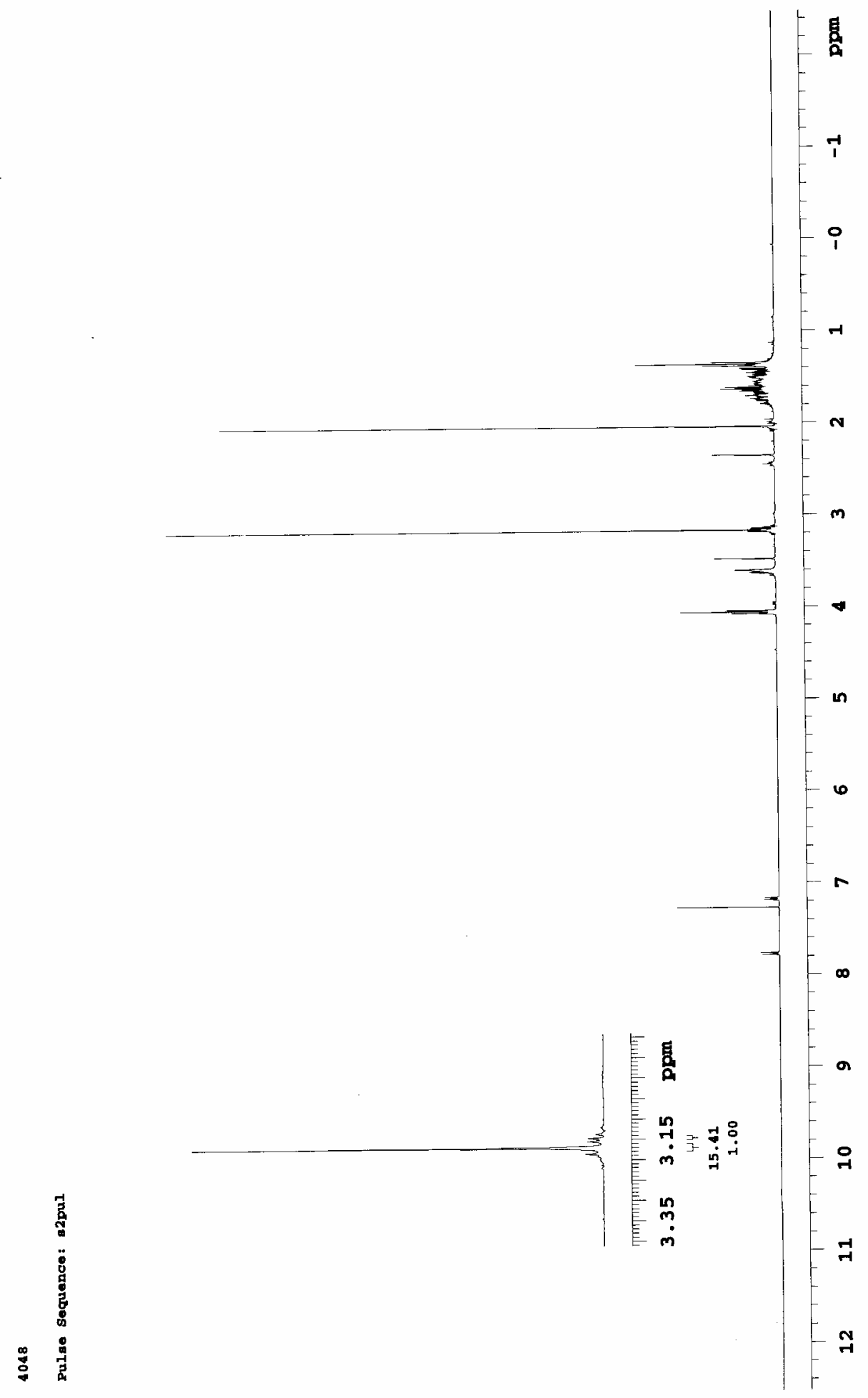


${ }^{1}$ H NMR-spectrum for Table 2, entry 5 (400 MHz)

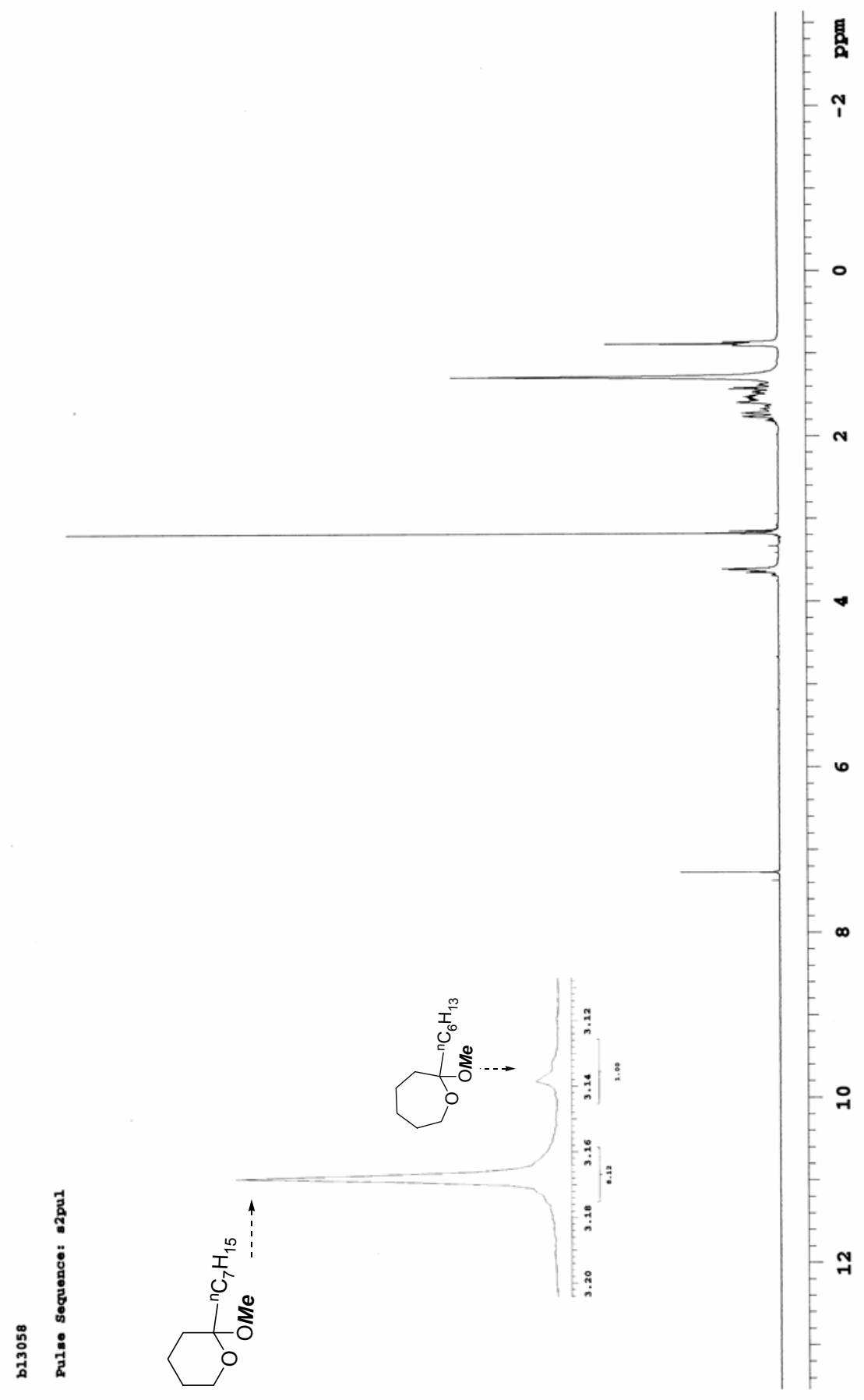


${ }^{1}$ H NMR-spectrum for Table 2, entry 6 (400 MHz)

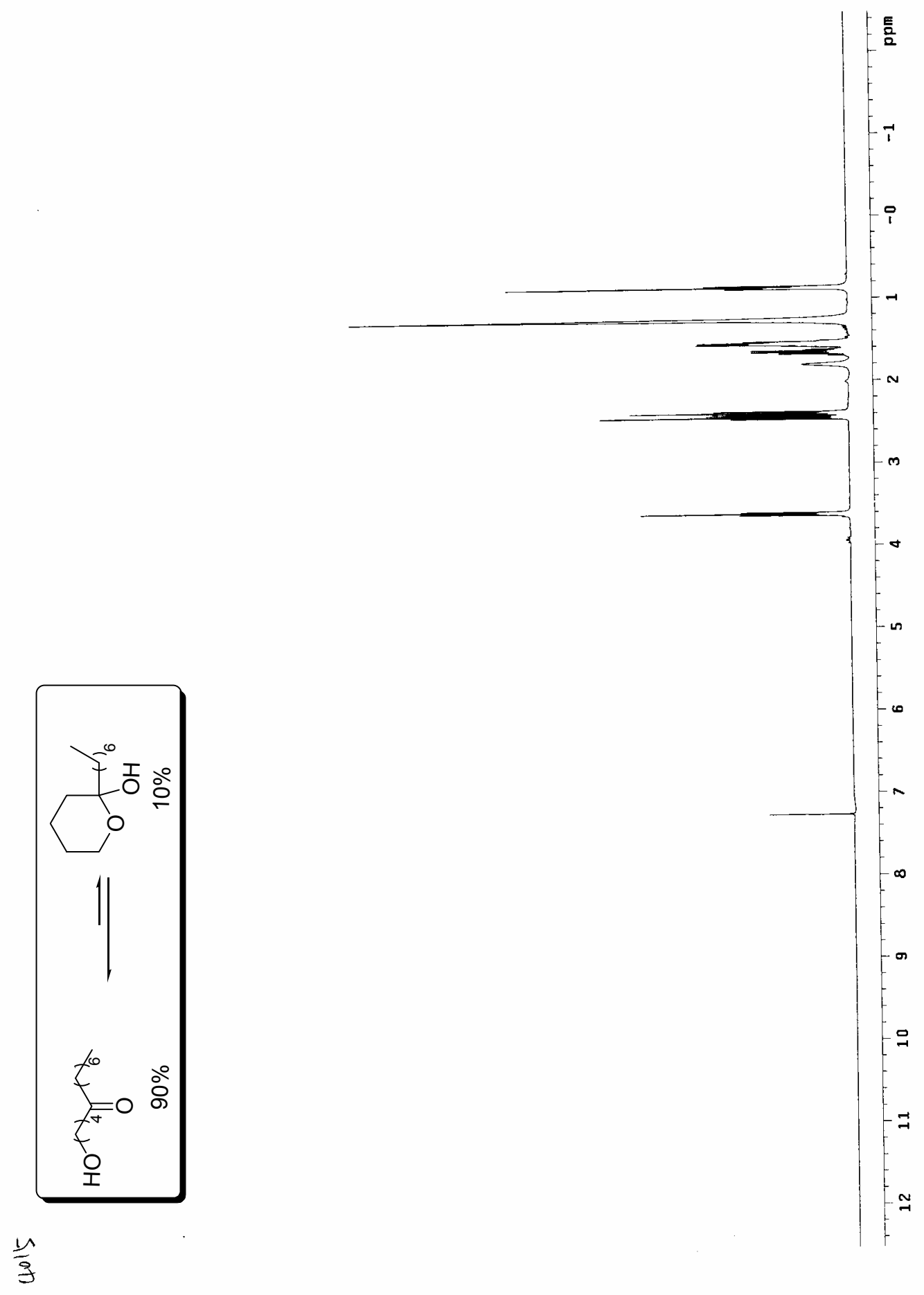


${ }^{13} \mathrm{C}$ NMR-spectrum for Table 2, entry 6 (100 MHz)

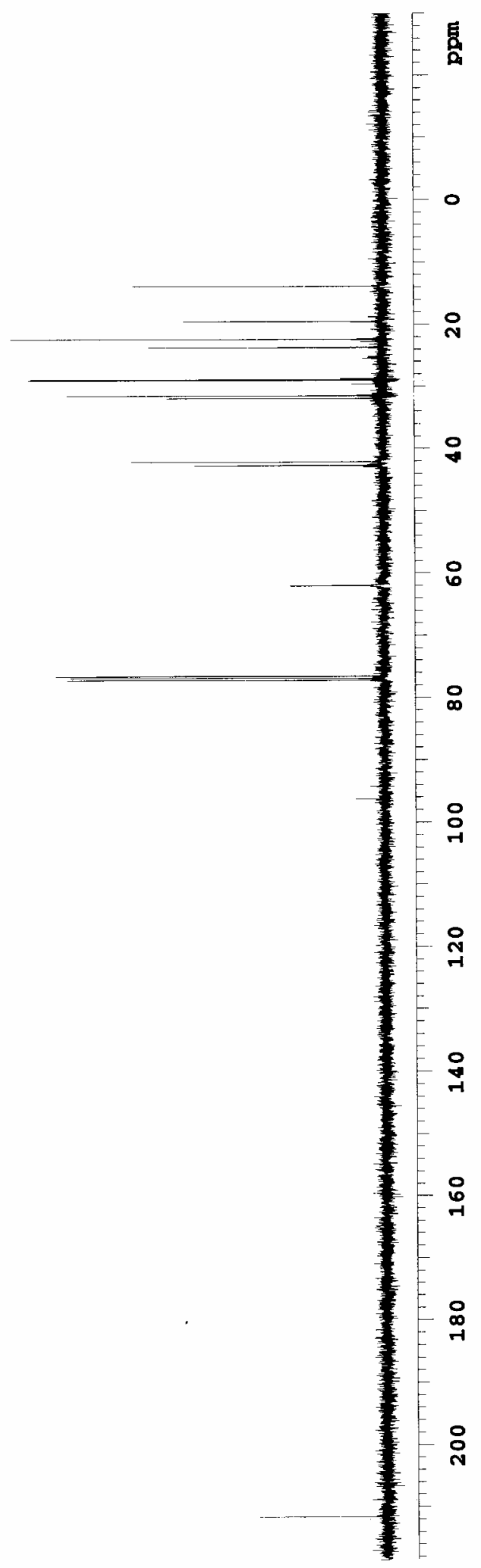


${ }^{1}$ H NMR-spectrum for Table 3, entry 4 (400 MHz)

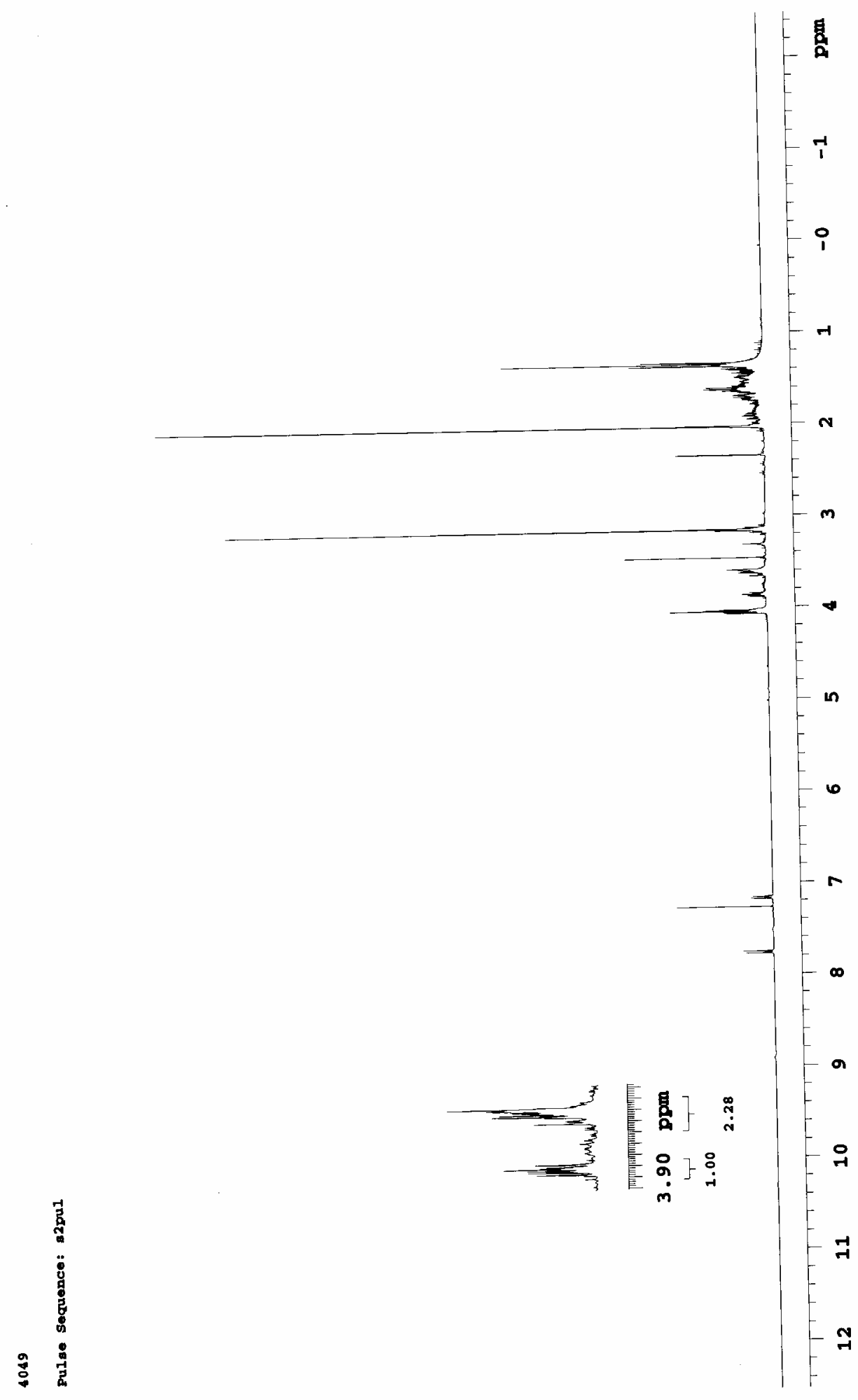


${ }^{1}$ H NMR-spectrum for Table 3, entry 5 (400 MHz)

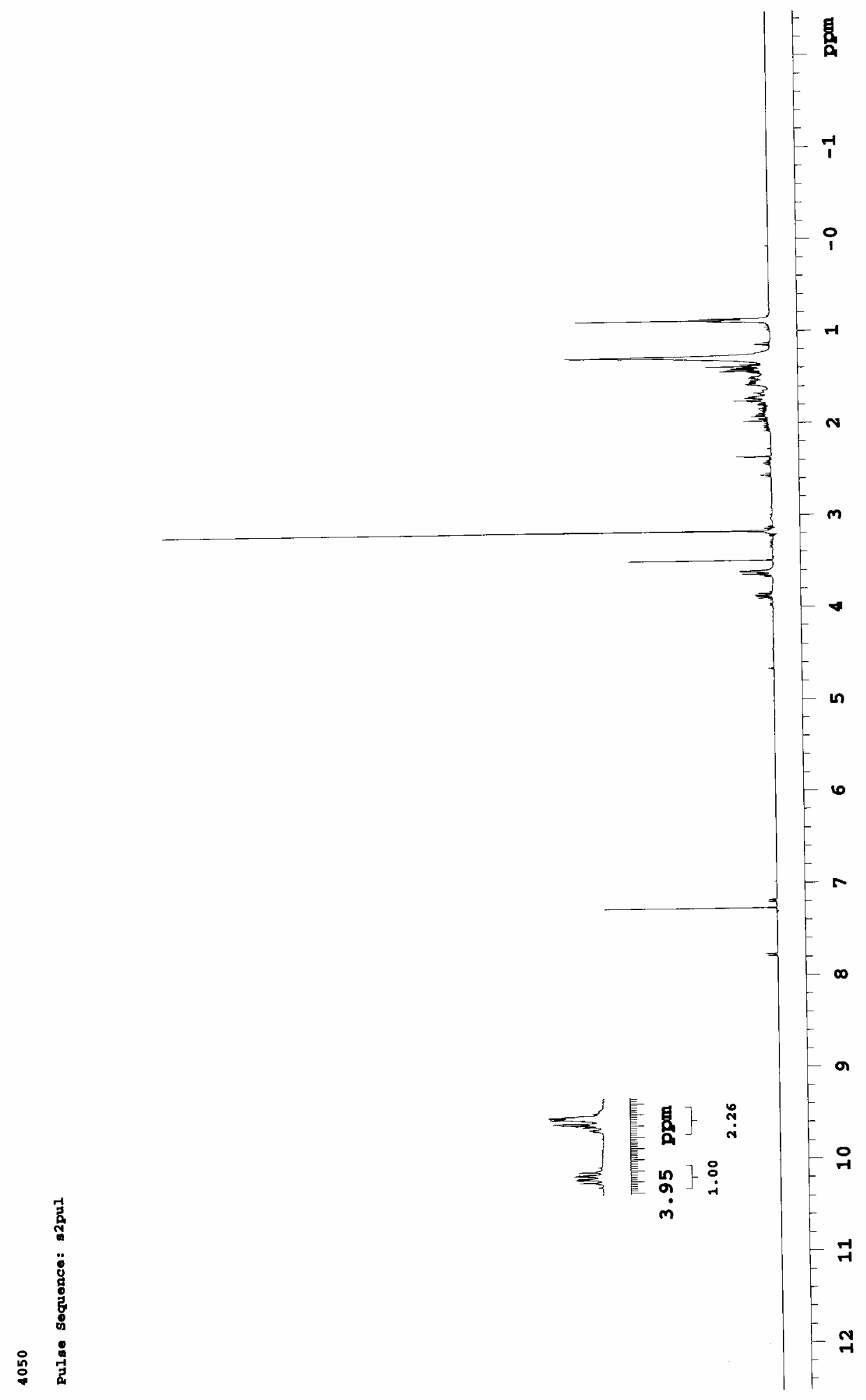


${ }^{1}$ H NMR-spectrum for Table 3, entry 6 (400 MHz)

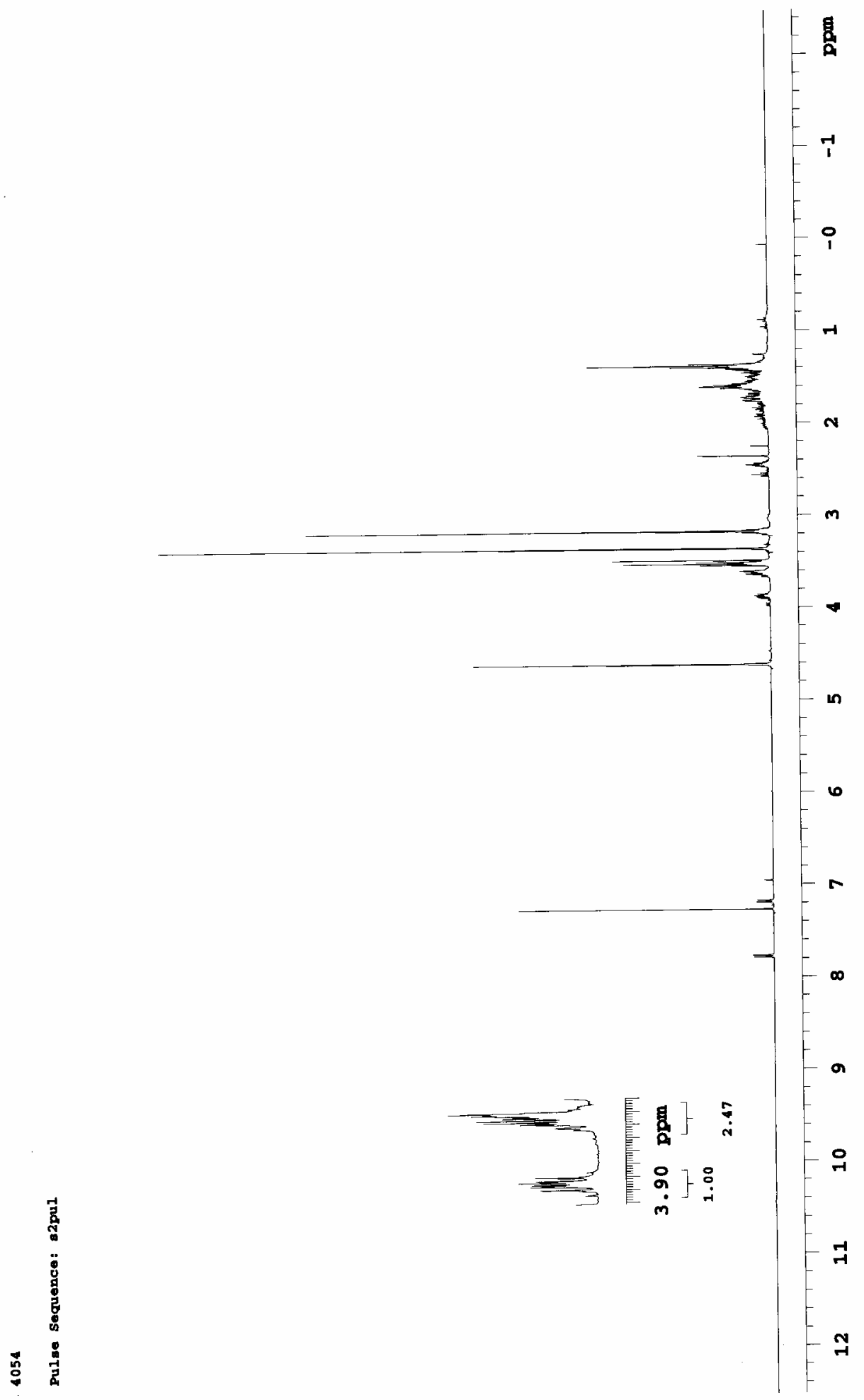


${ }^{1}$ H NMR-spectrum for Table 3, entry 7 (400 MHz)

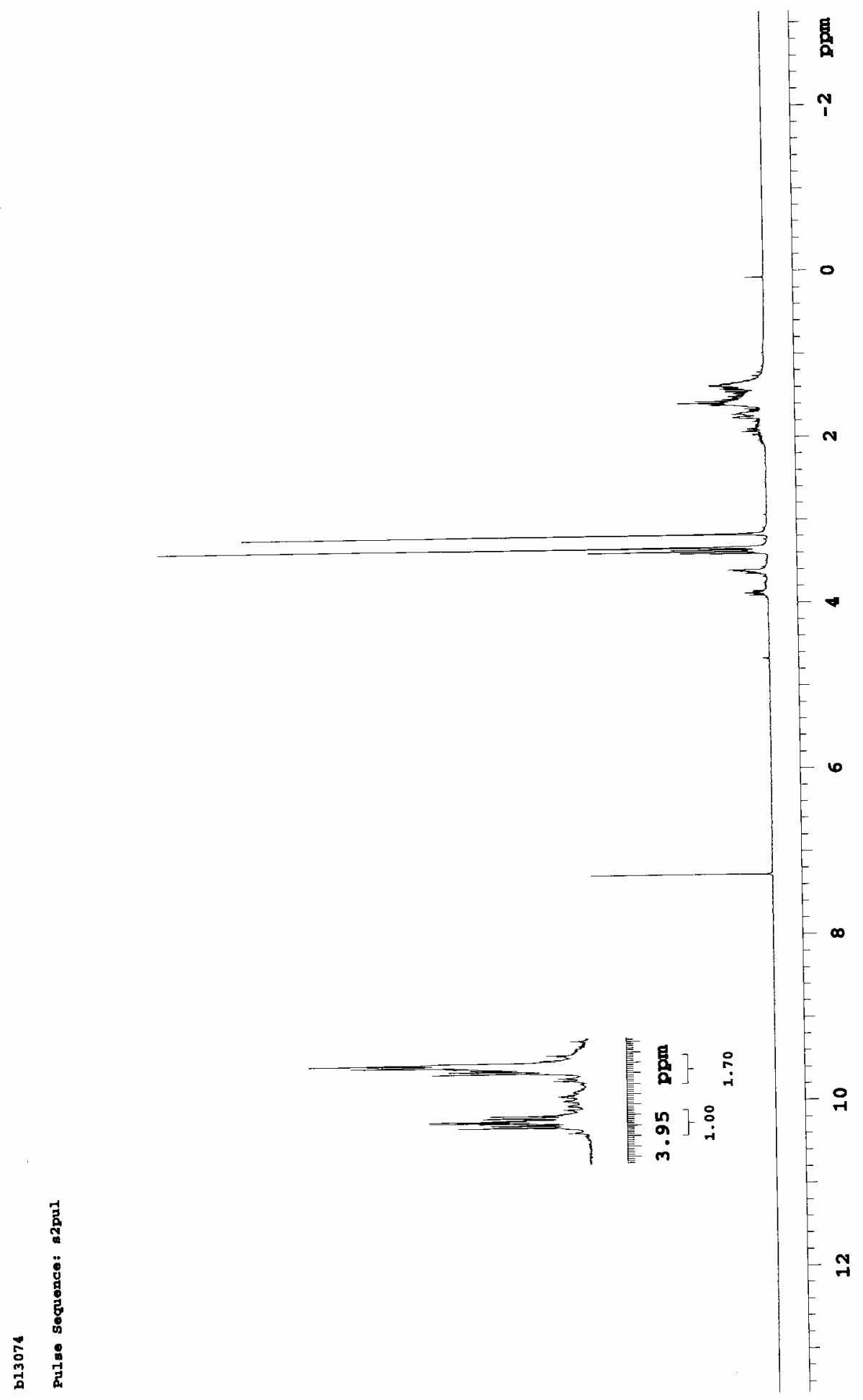


${ }^{1}$ H NMR-spectrum for Table 3, entry 8 (400 MHz)

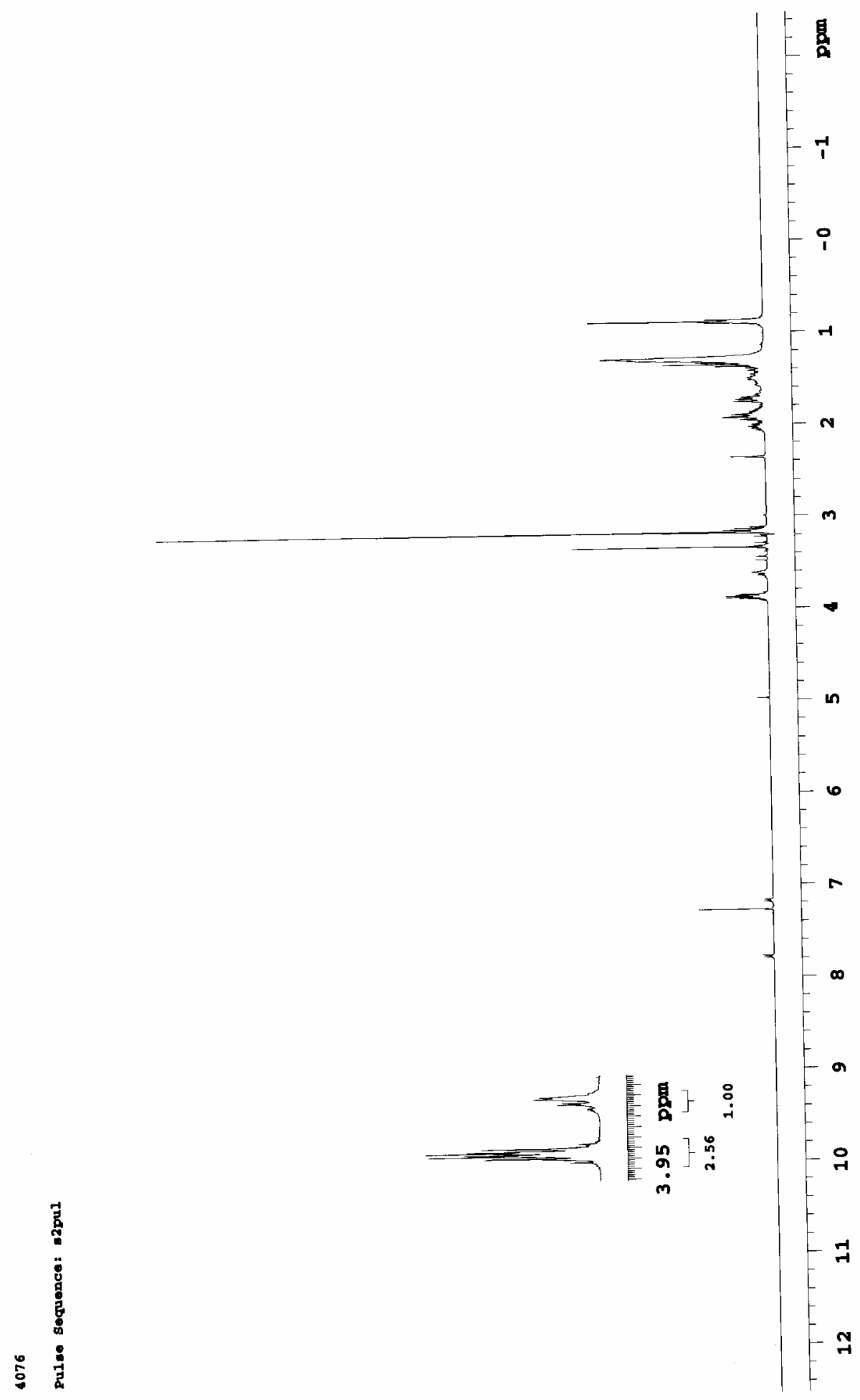


${ }^{1}$ H NMR-spectrum for Table 3, entry 10 (400 MHz)

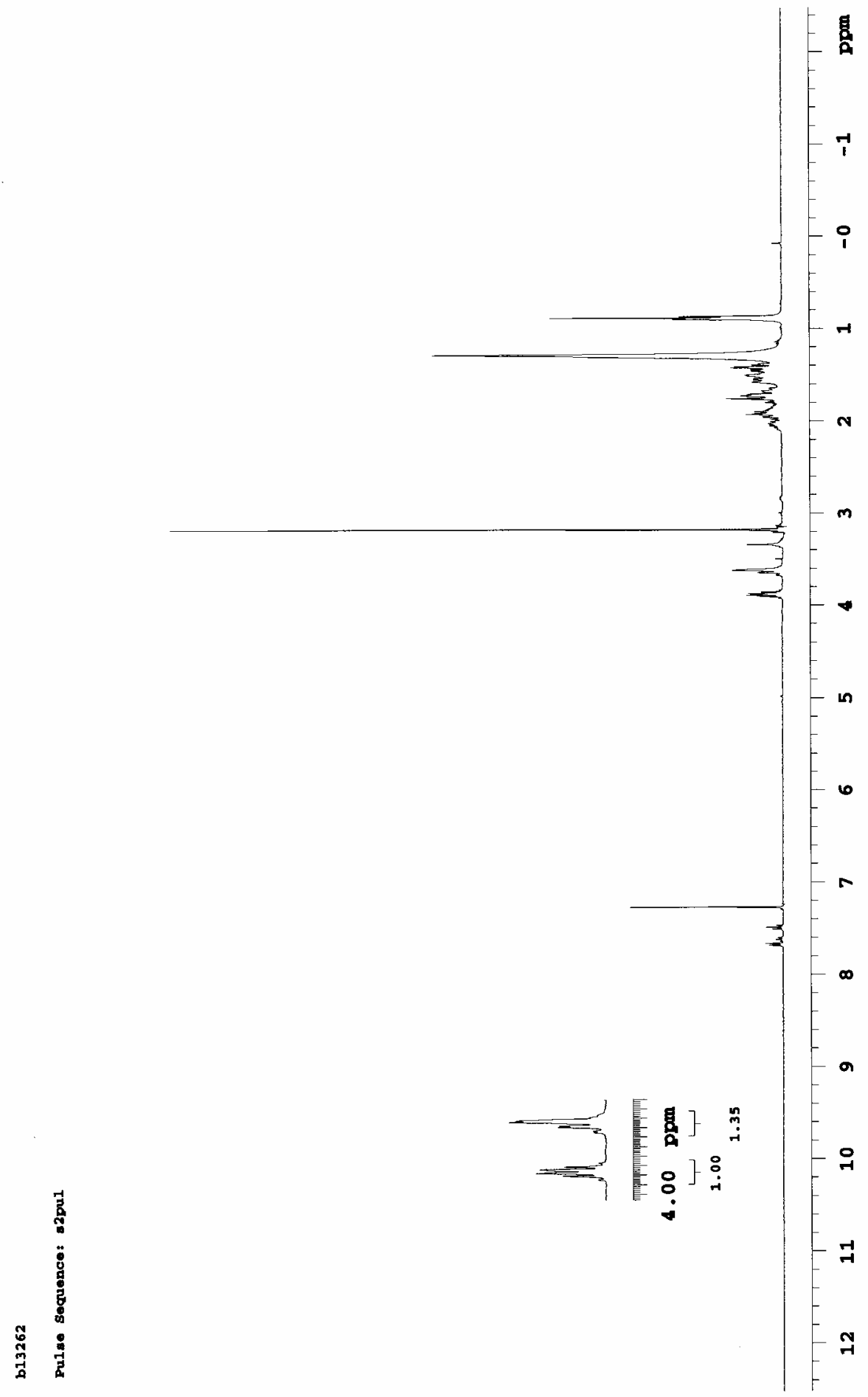


${ }^{1} \mathrm{H}$ NMR-spectrum for Table 3, entry 13 (400 MHz)

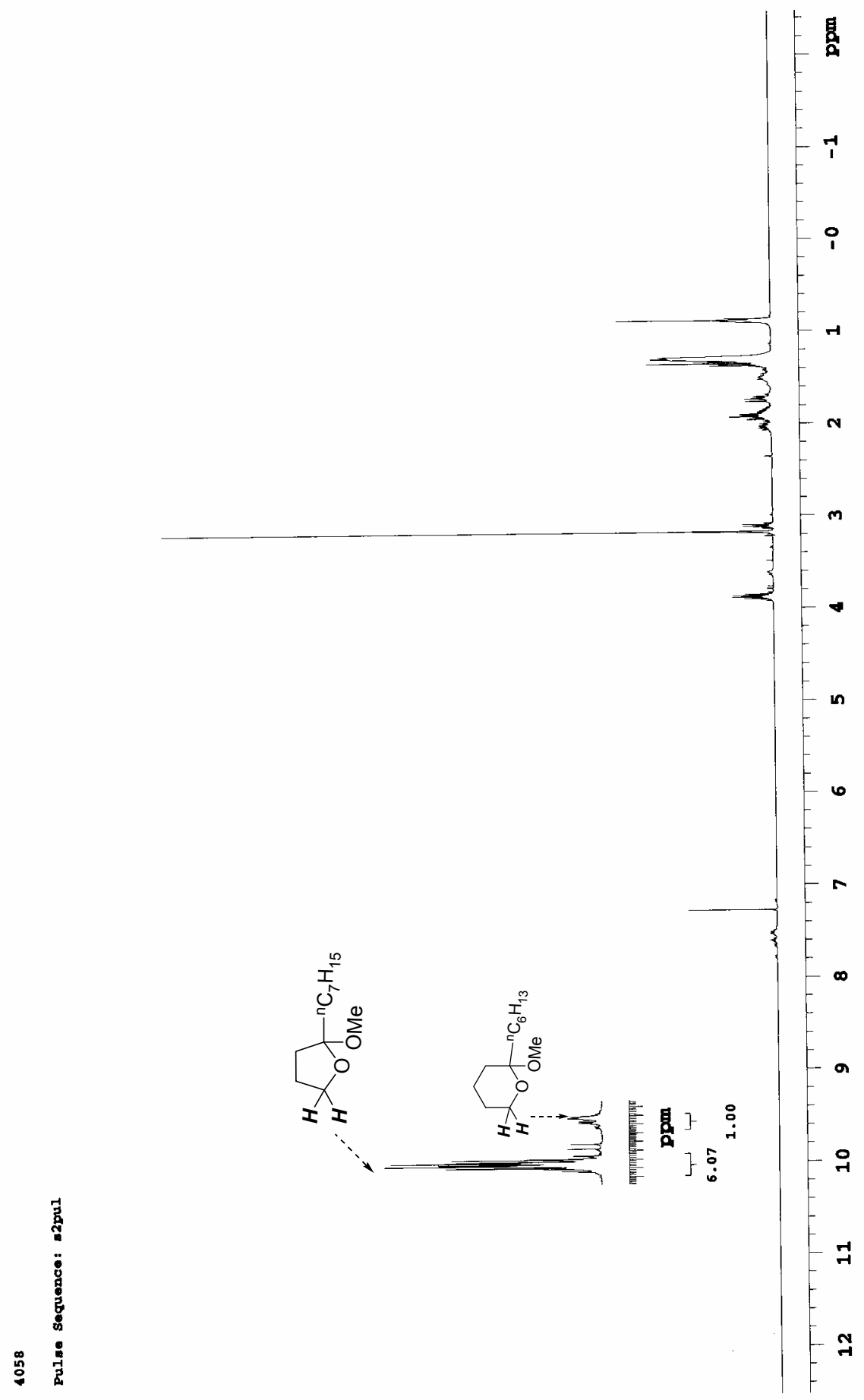


${ }^{1}$ H NMR-spectrum for Table 3, entry 14 (400 MHz)

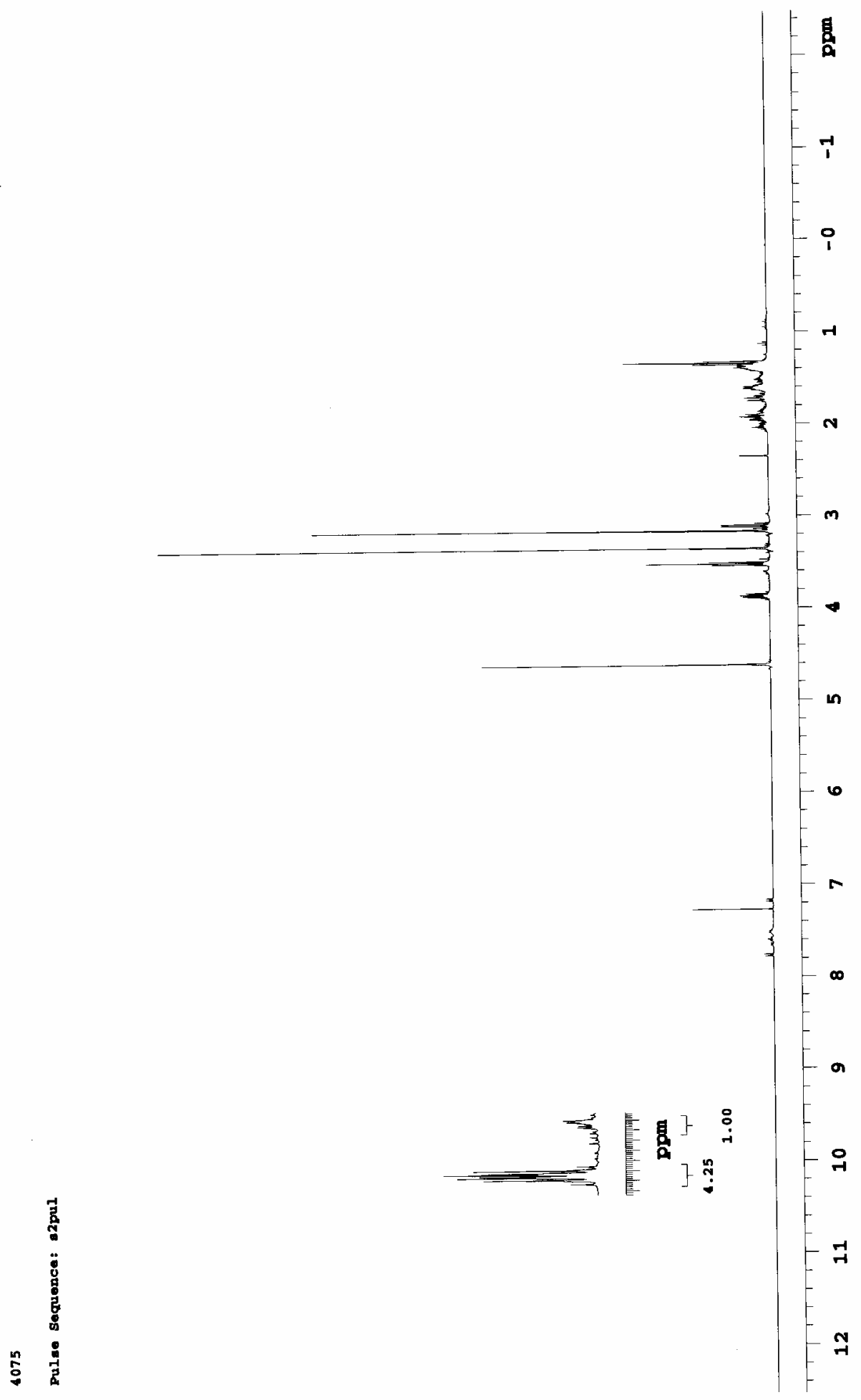


${ }^{1}$ H NMR for Table 4, entry $1\left(400 \mathrm{MHz}, \mathrm{CD}_{3} \mathrm{OD}\right)$

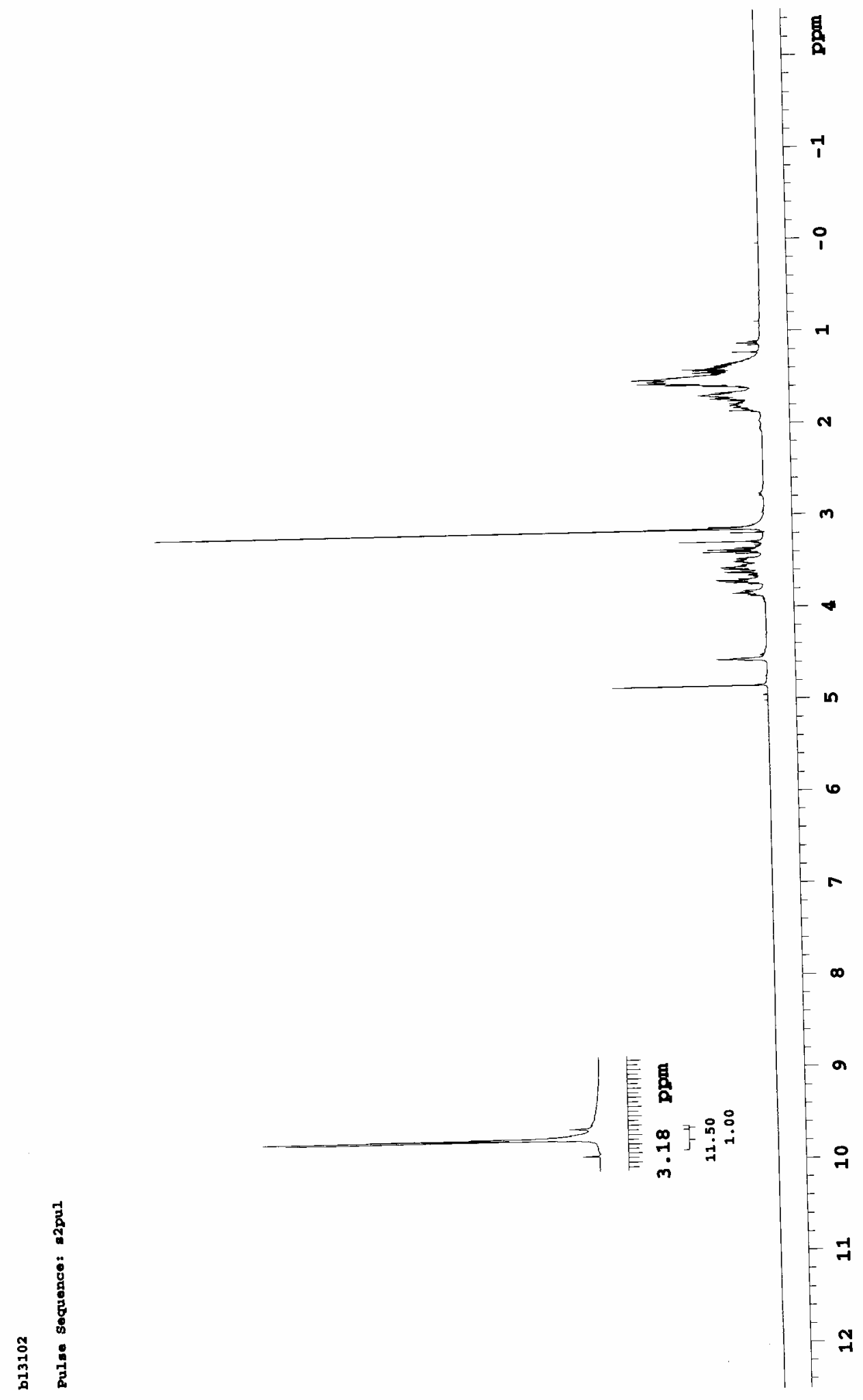


${ }^{13}$ C NMR for Table 4, entry 1 (100 MHz)

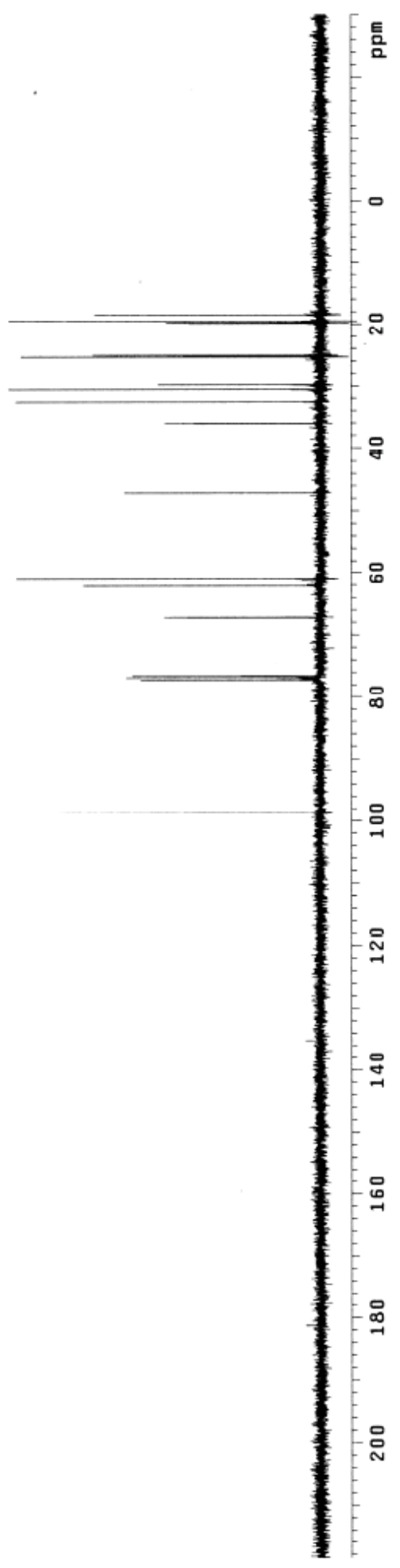


${ }^{1}$ H NMR for Table 4, entry 2 (300 MHz)

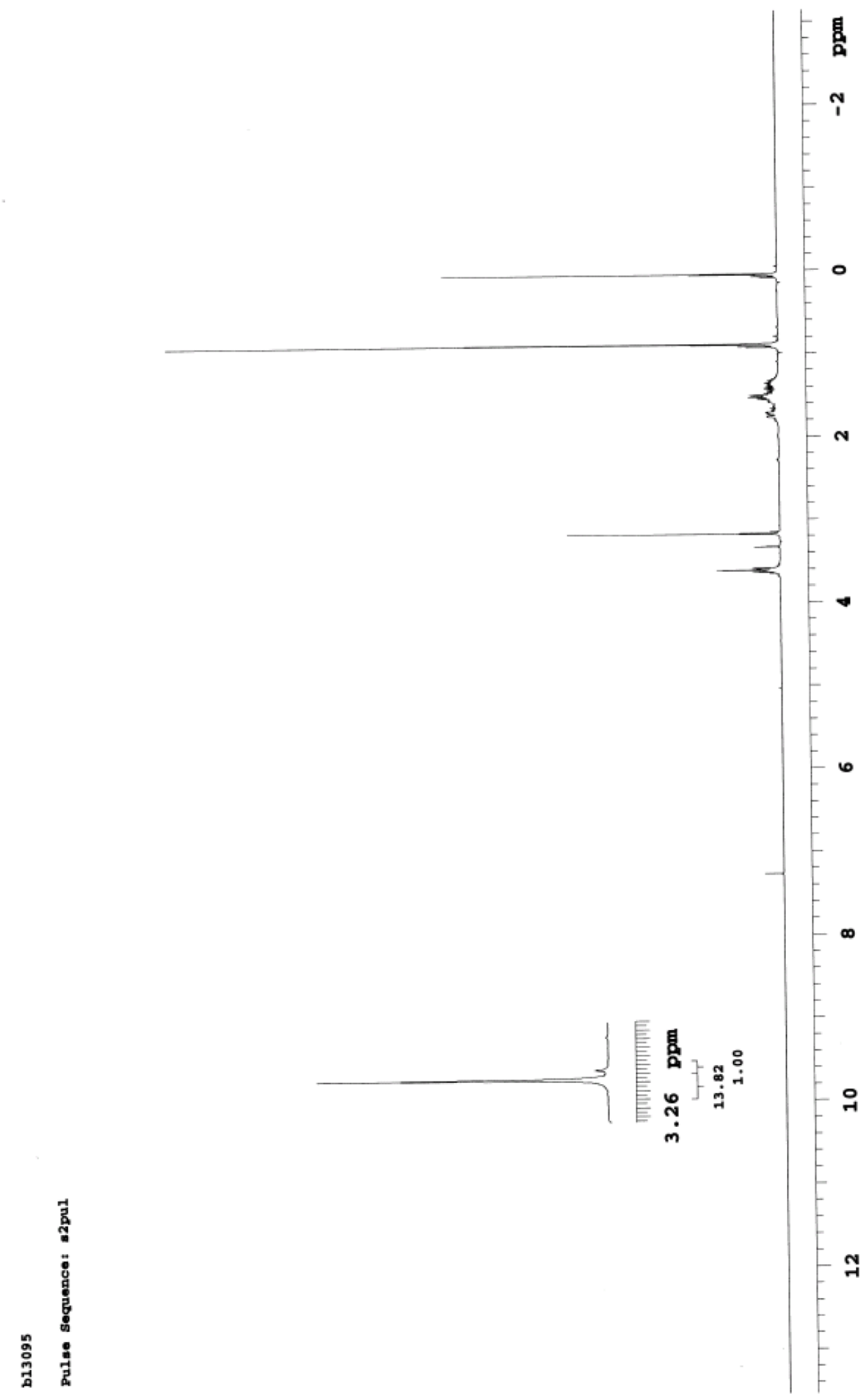


${ }^{13} \mathrm{C}$ NMR for Table 4, entry 2 (75 MHz)

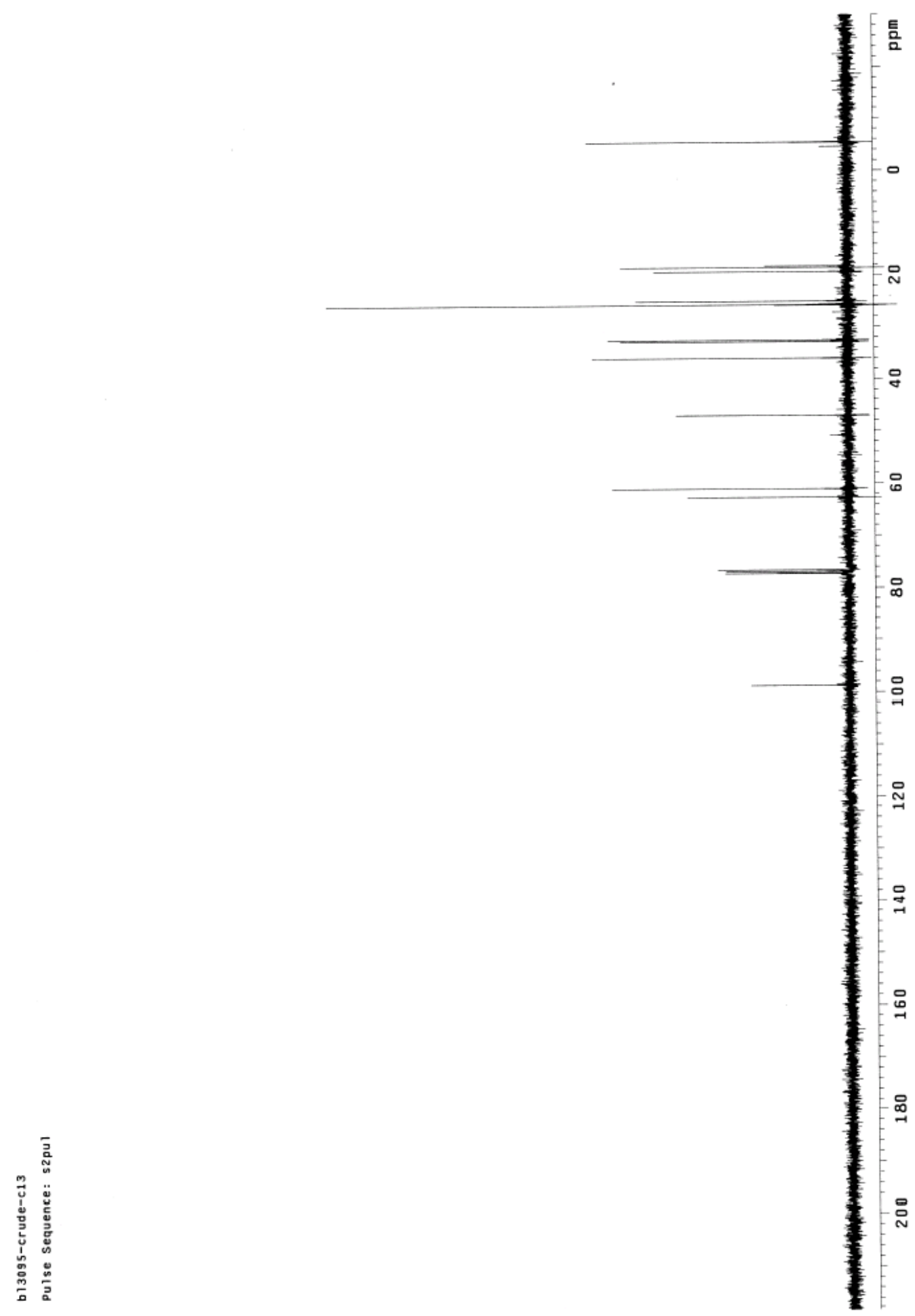


${ }^{1}$ H NMR for Table 4, entry 3 (400 MHz)

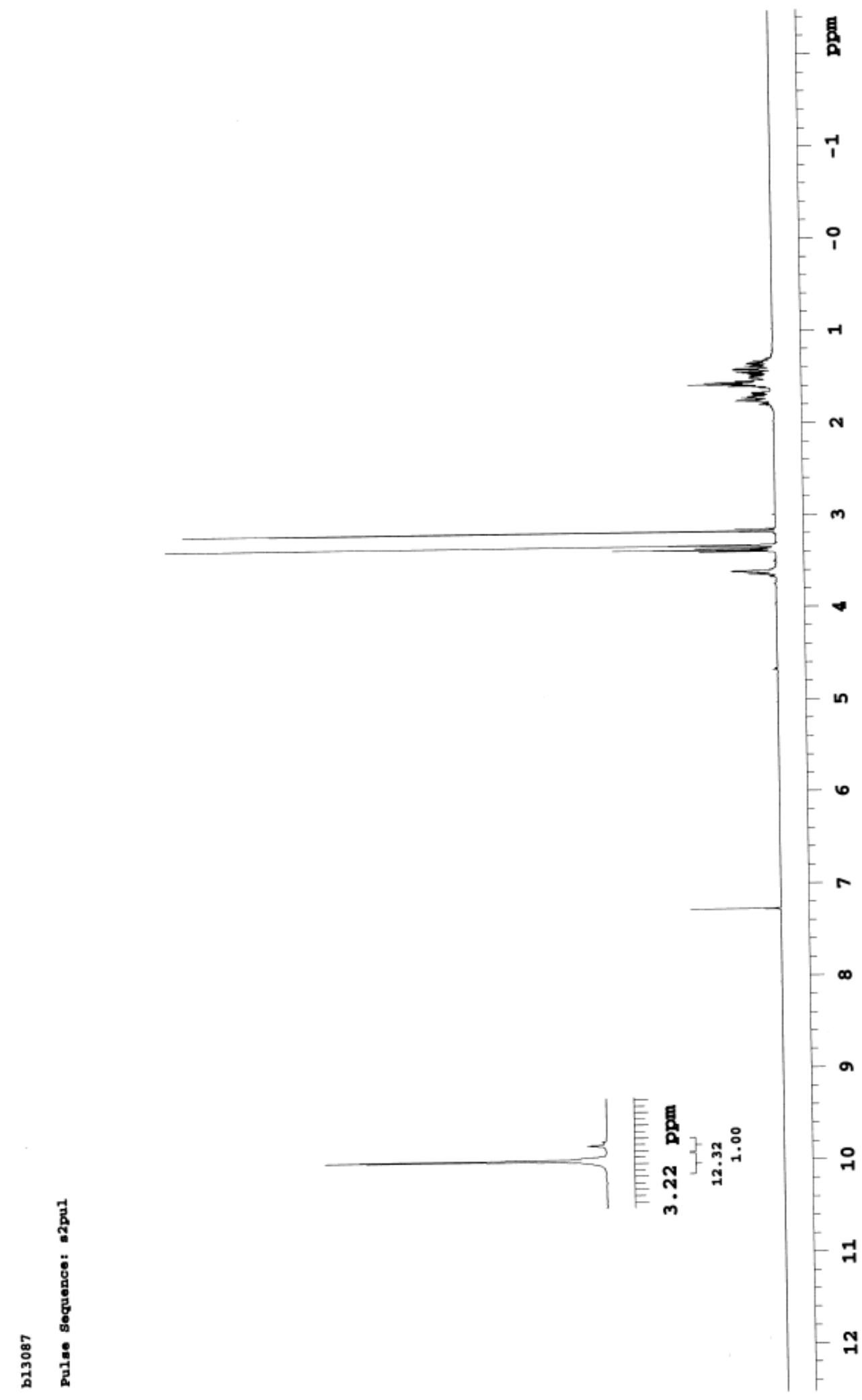


${ }^{13}$ C NMR for Table 4, entry $3(100 \mathrm{MHz})$

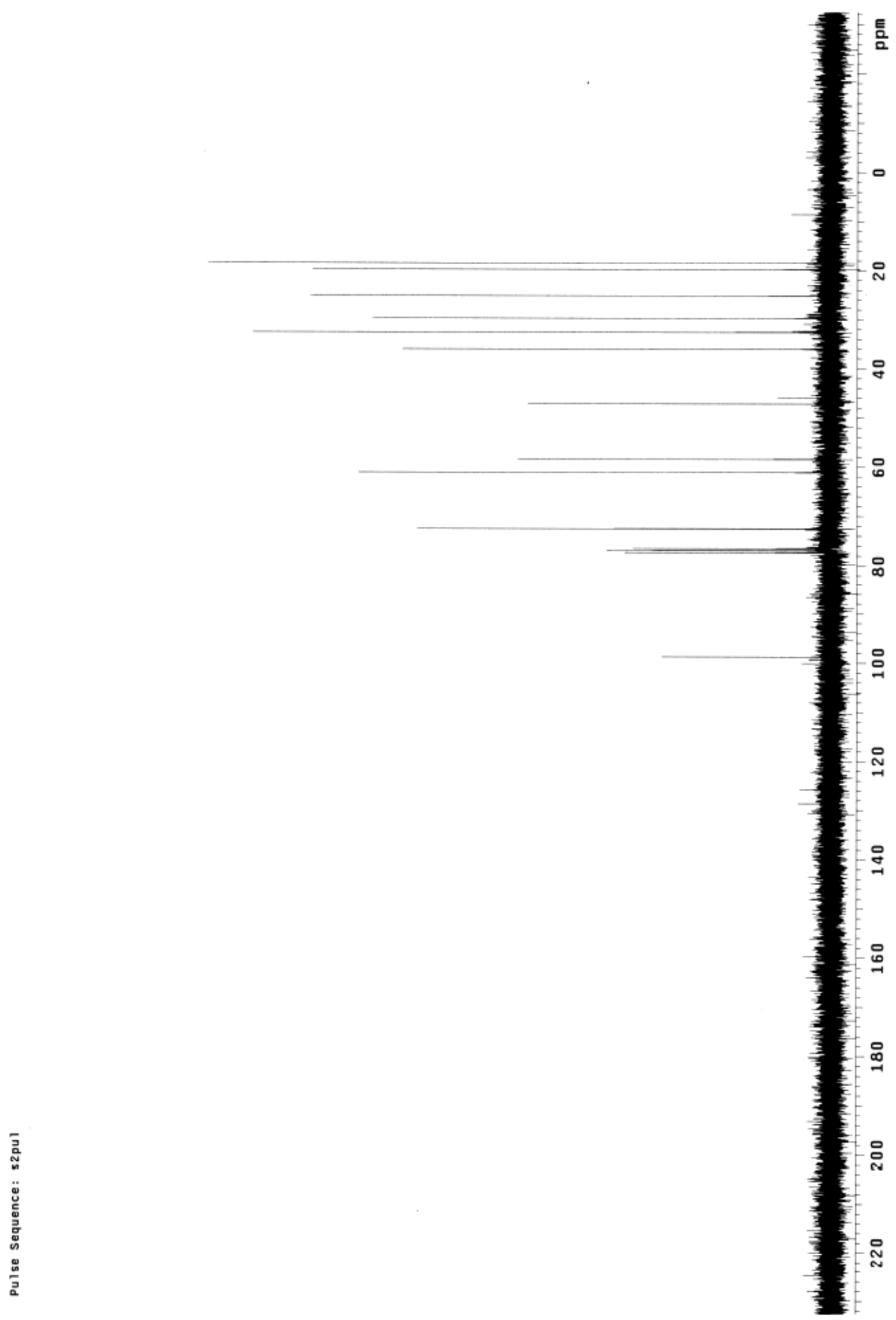


${ }^{1}$ H NMR for Table 4, entry 4 (400 MHz)

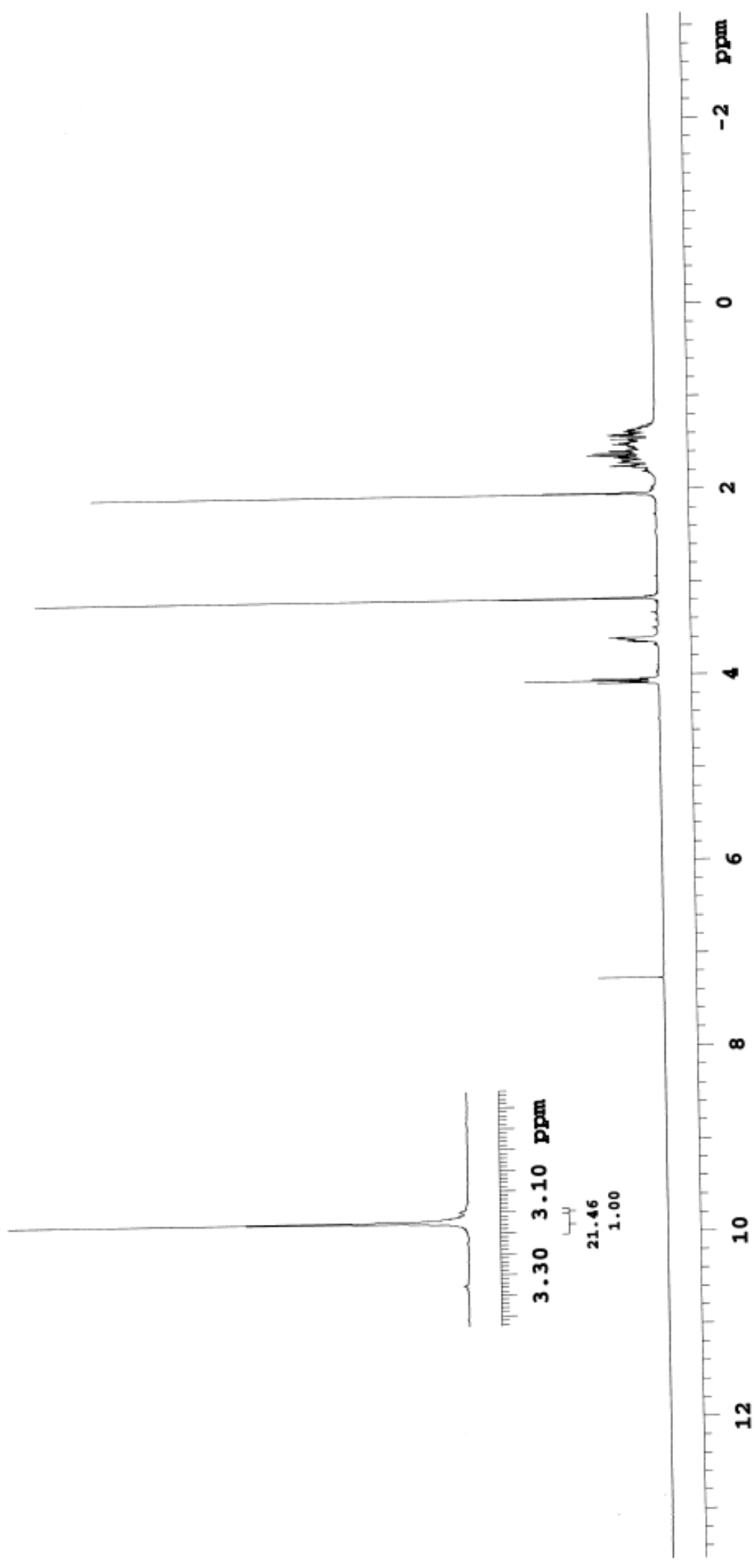


${ }^{13}$ C NMR for Table 4, entry 4 (100 MHz)

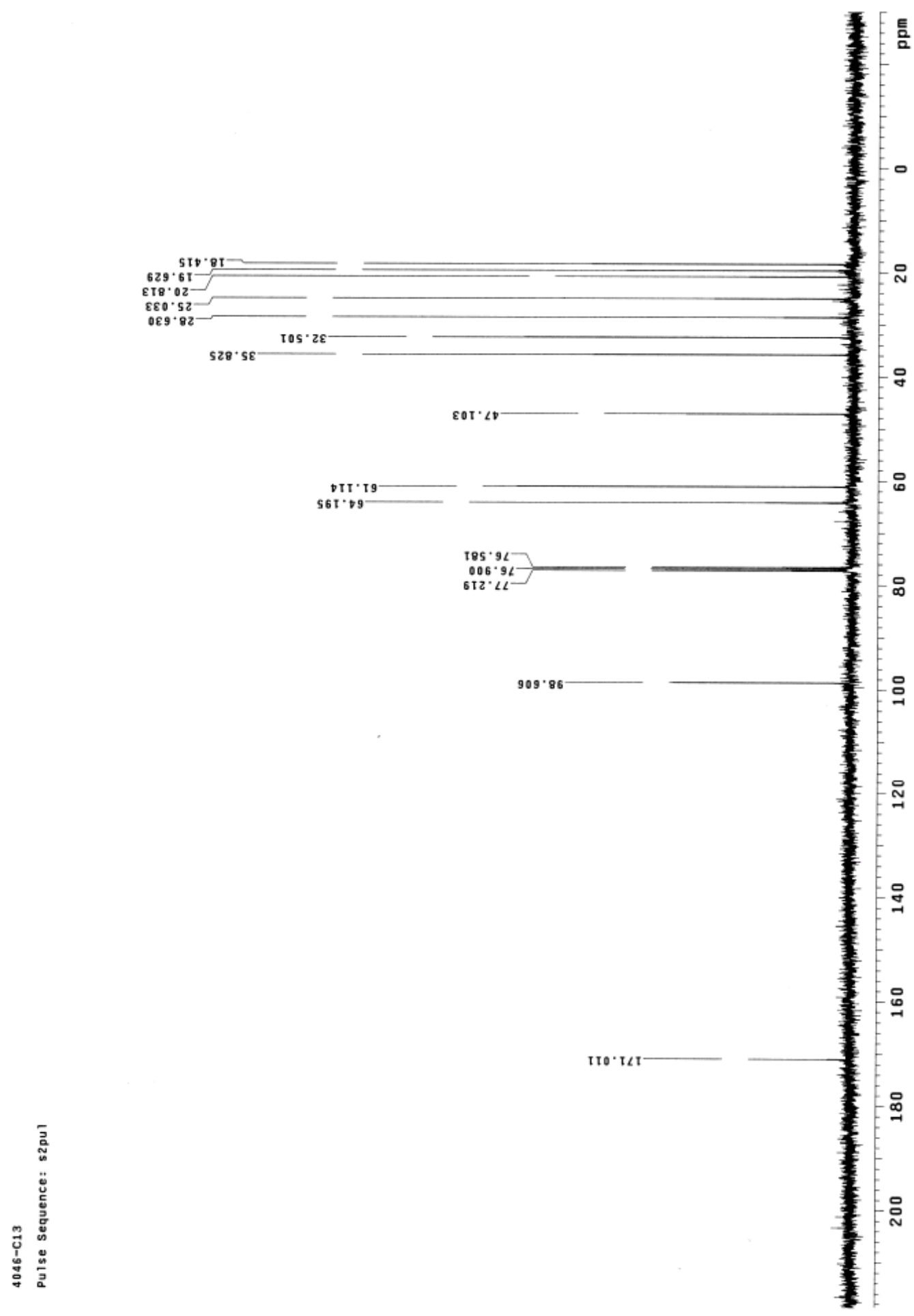


${ }^{1}$ H NMR for Table 4, entry 5 (400 MHz)

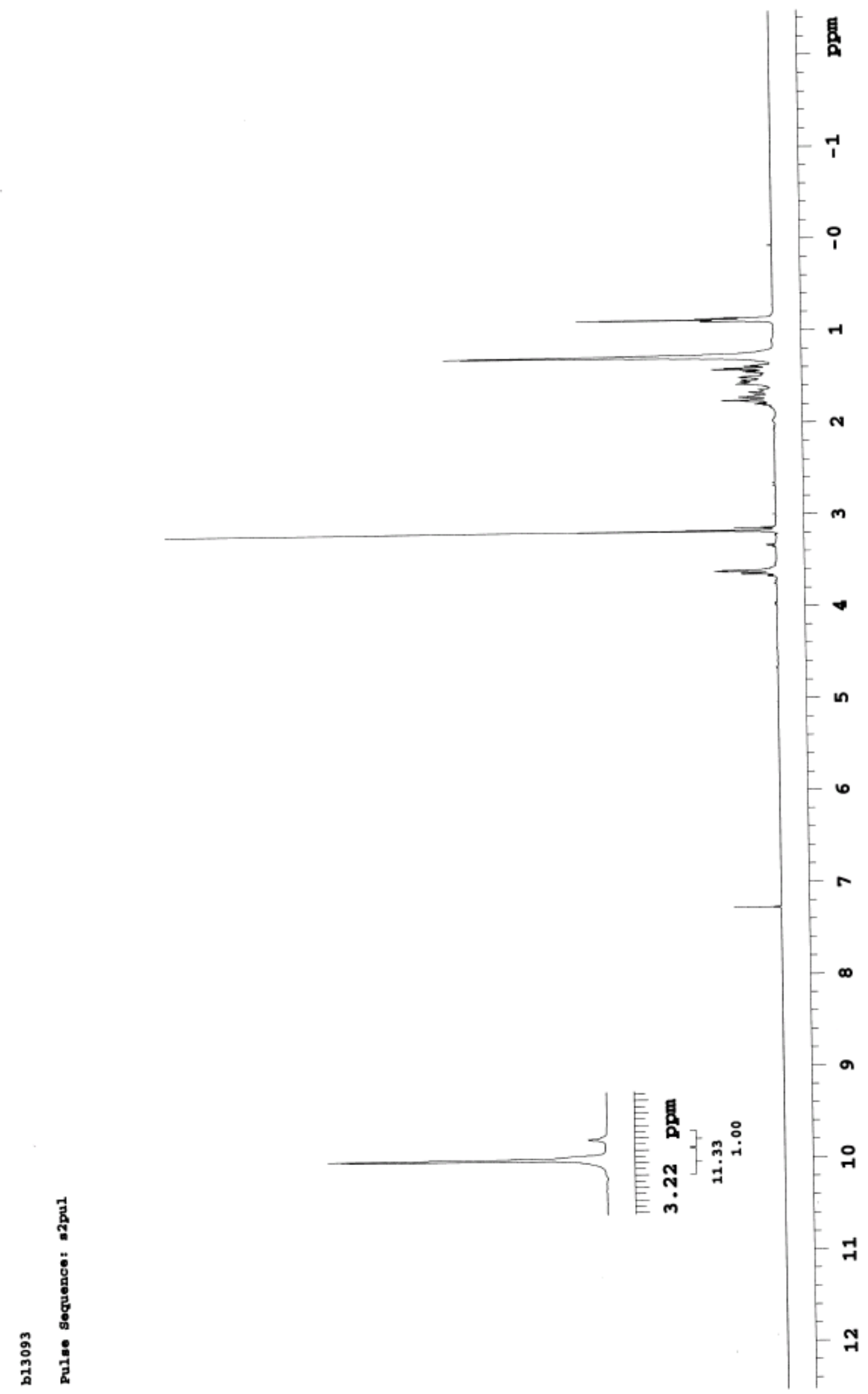


${ }^{13}$ C NMR for Table 4, entry 5 (100 MHz)

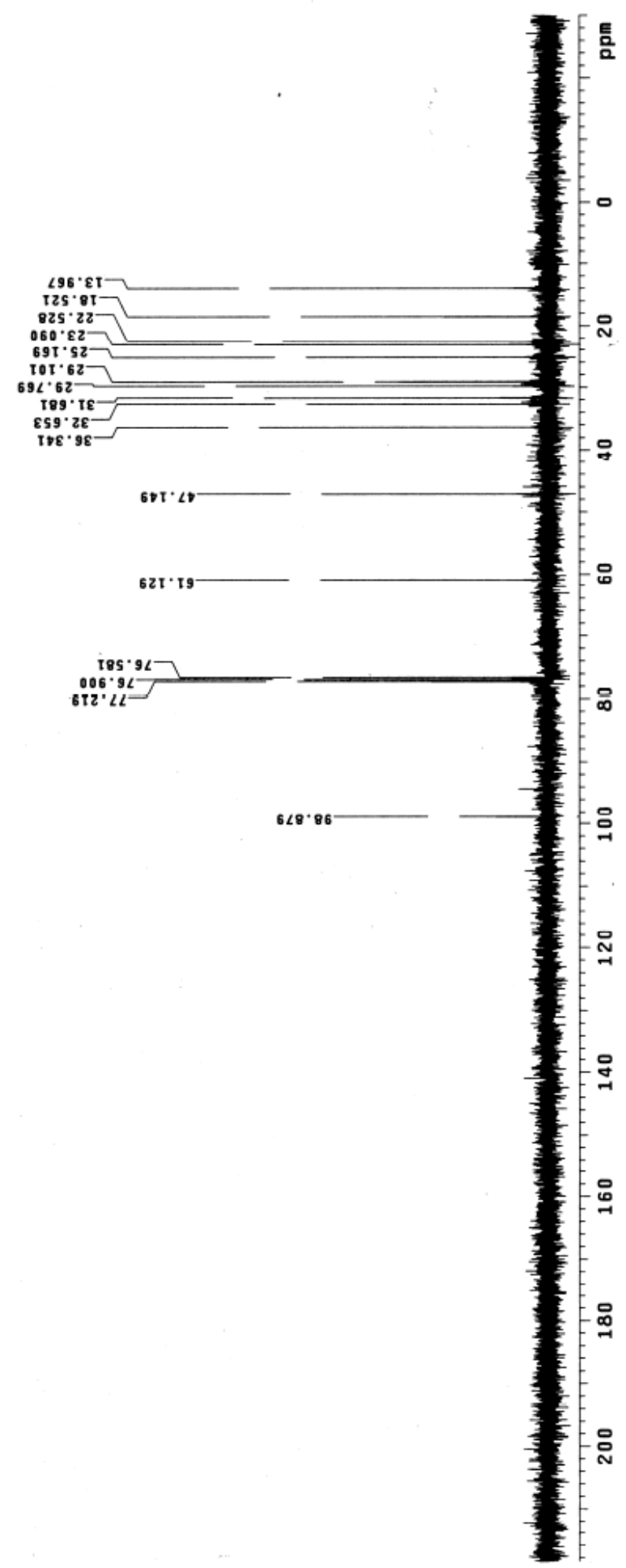


${ }^{1}$ H NMR for Table 4, entry 6 (300 MHz)

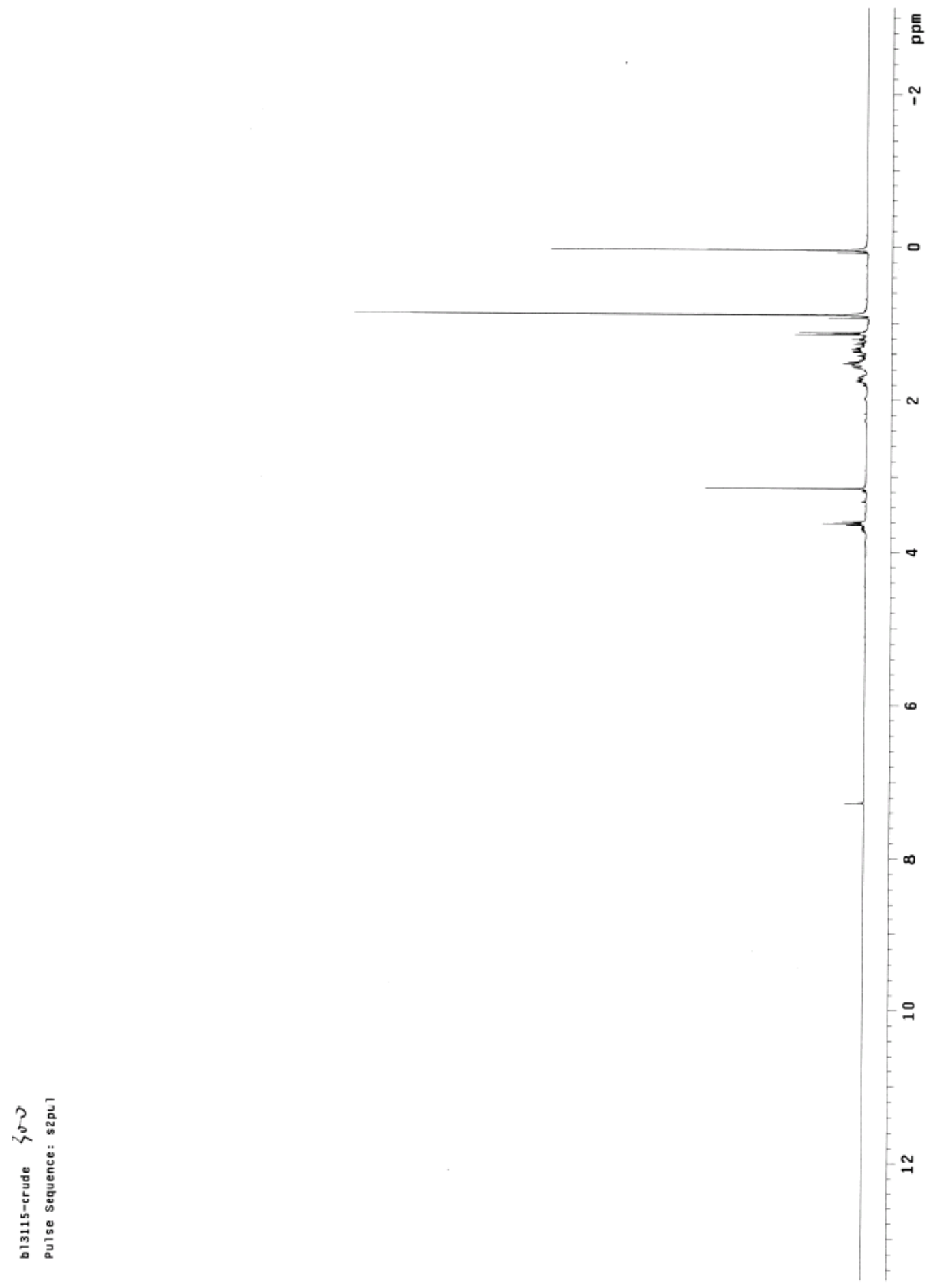


${ }^{13} \mathrm{C}$ NMR for Table 4, entry $6(75 \mathrm{MHz})$

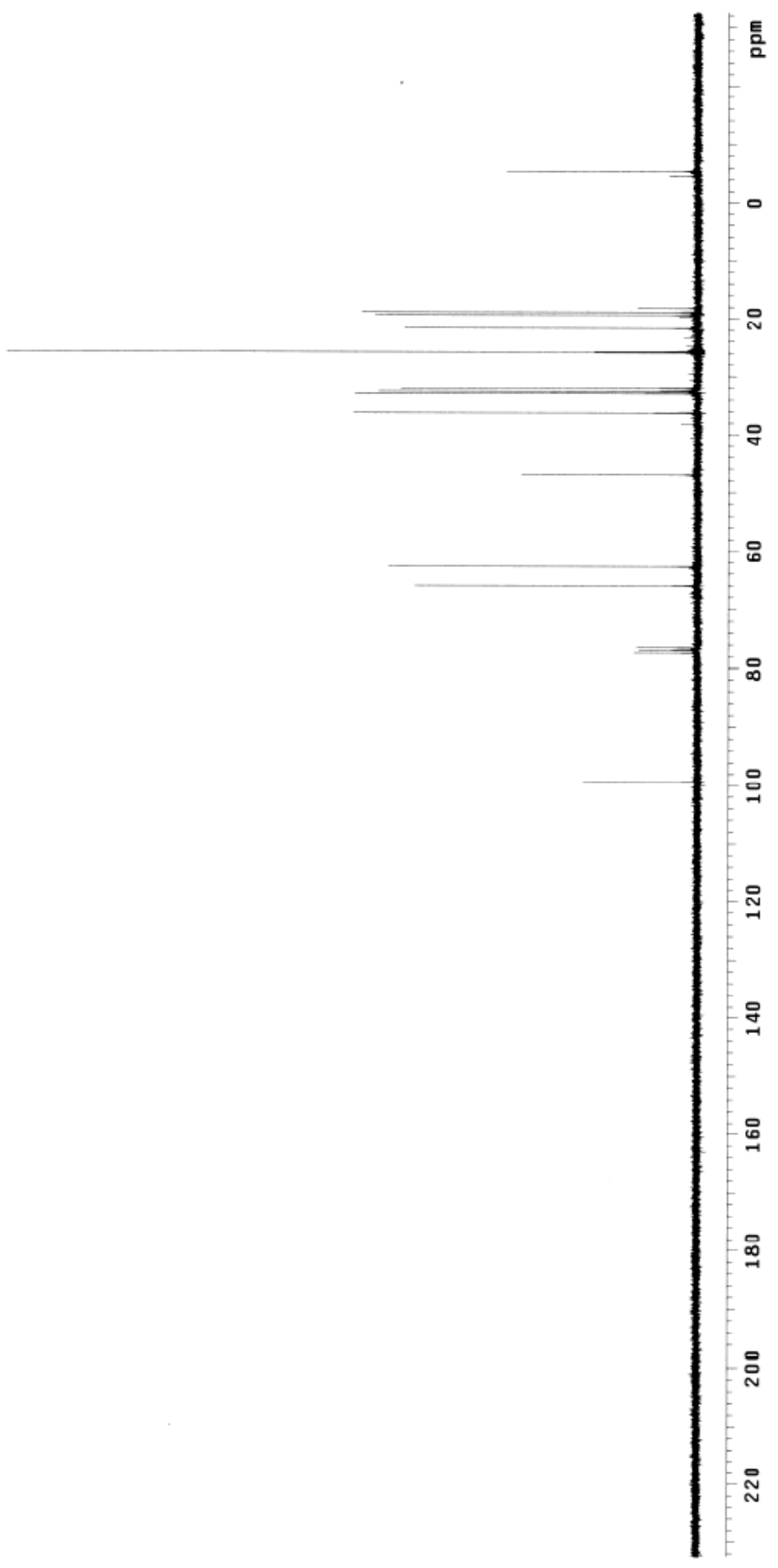


${ }^{1}$ H NMR for Table 4, entry 7 (400 MHz)

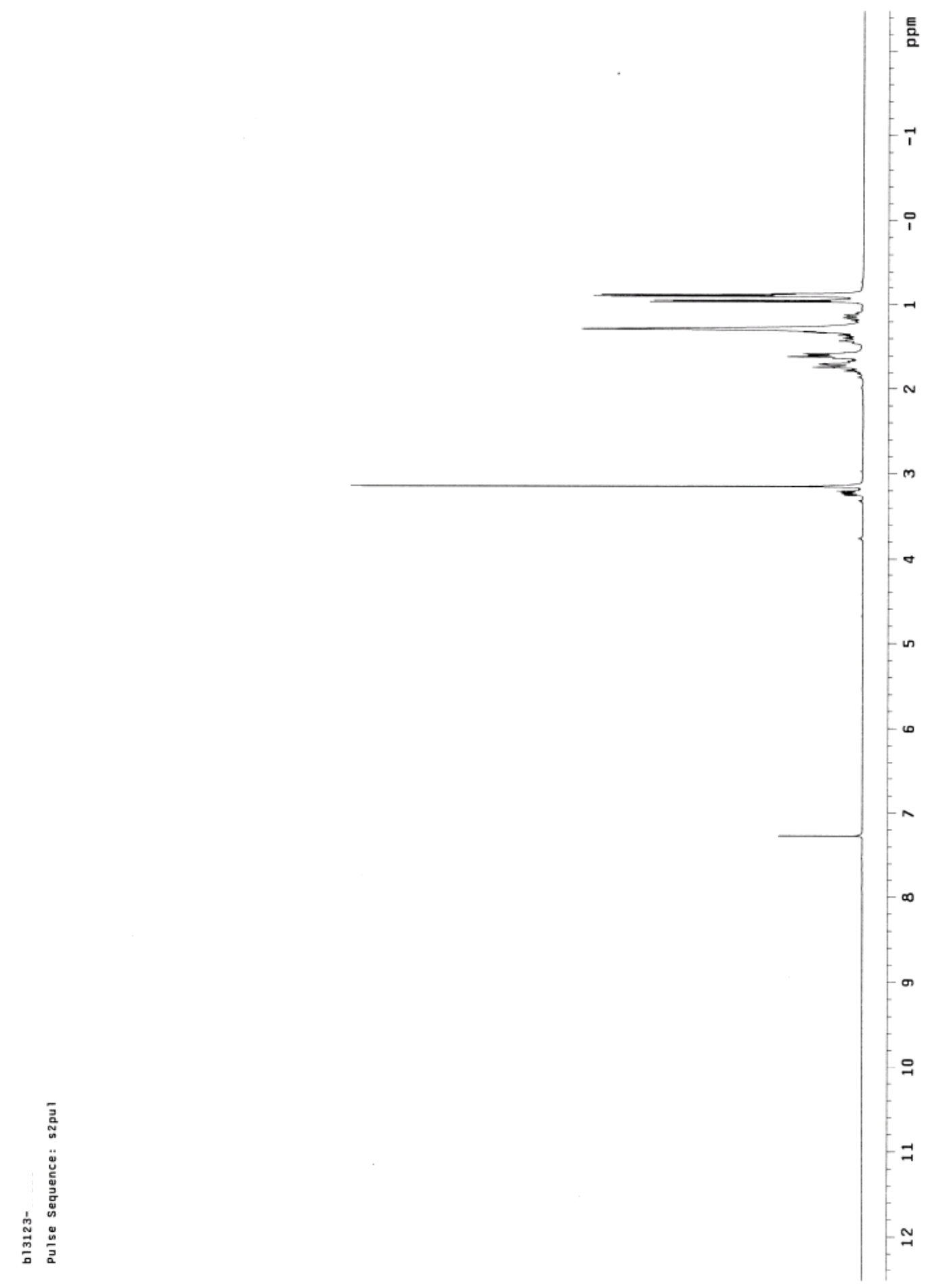


${ }^{13}$ C NMR for Table 4, entry 7 (100 MHz)

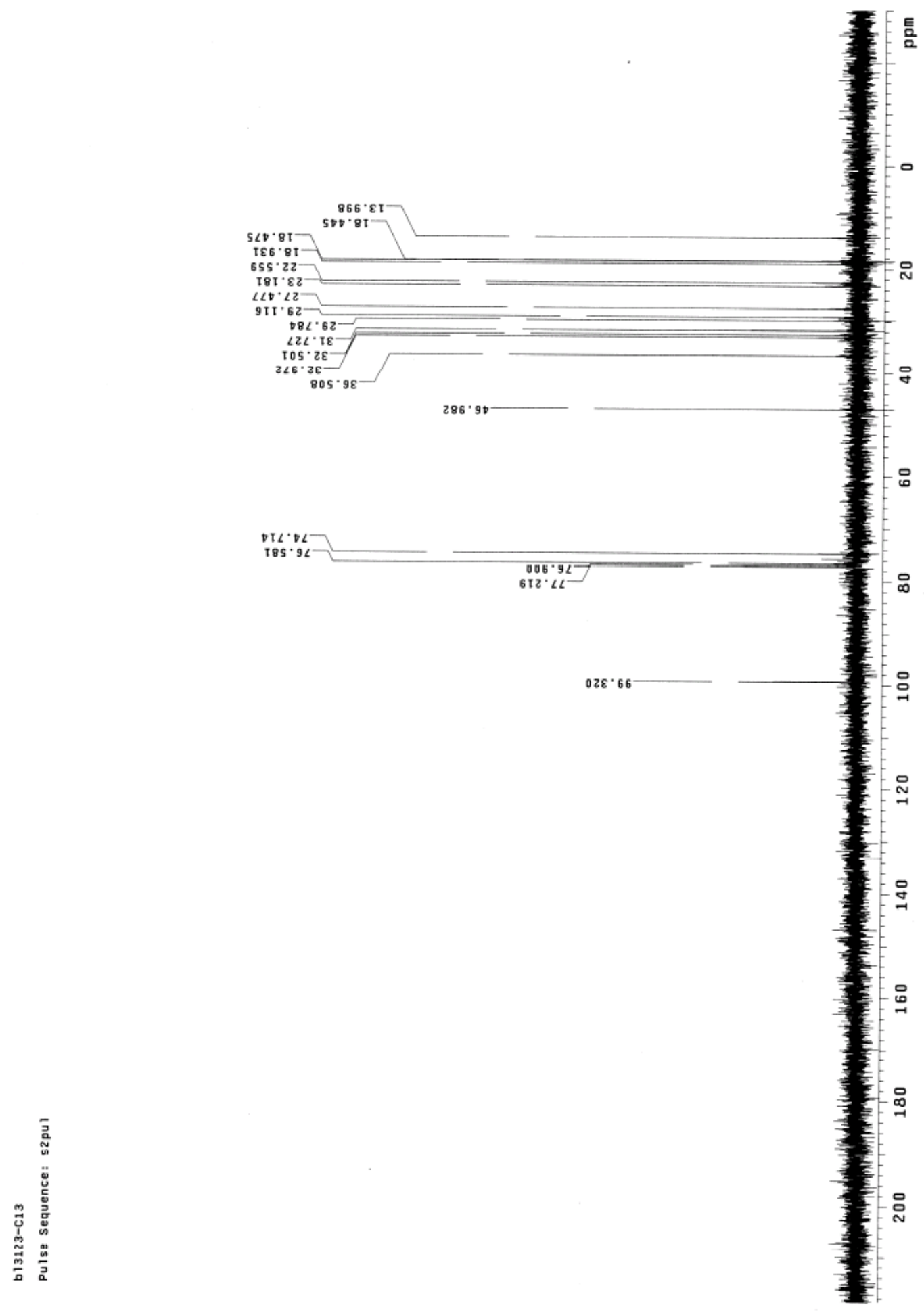


${ }^{1}$ H NMR for Table 4, entry 8 (300 MHz)

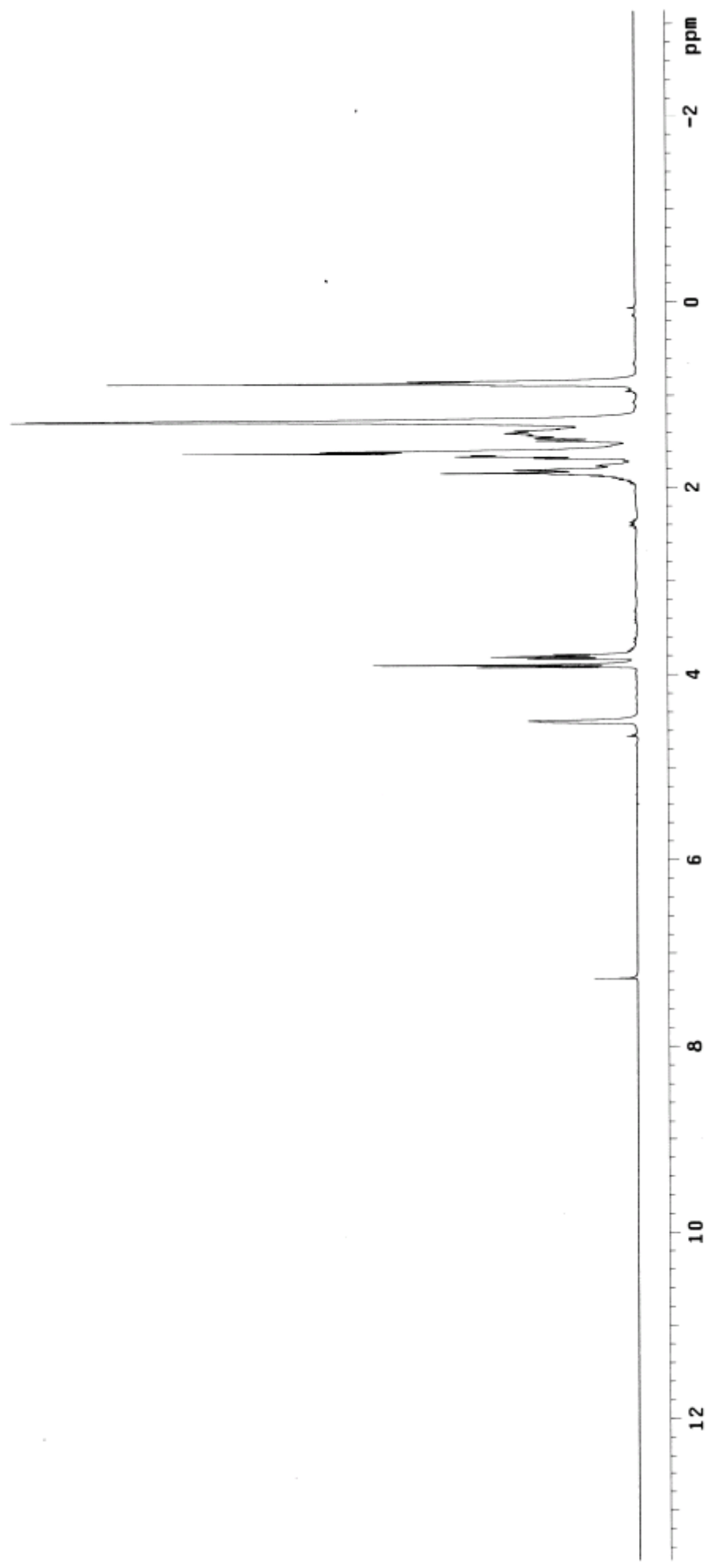


${ }^{13}$ C NMR for Table 4, entry 8 (100 MHz)

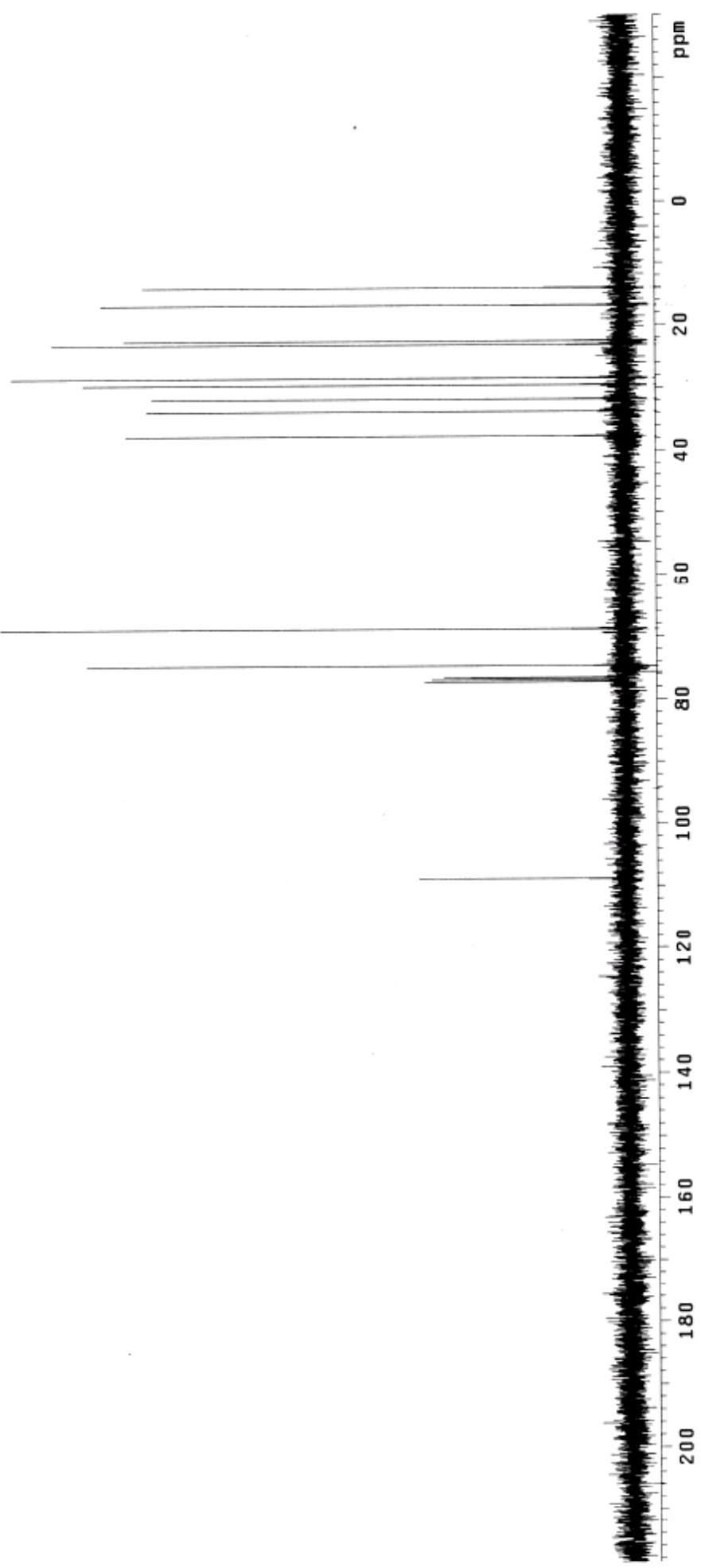


${ }^{1}$ H NMR for Table 4, entry $9(300 \mathrm{MHz})$

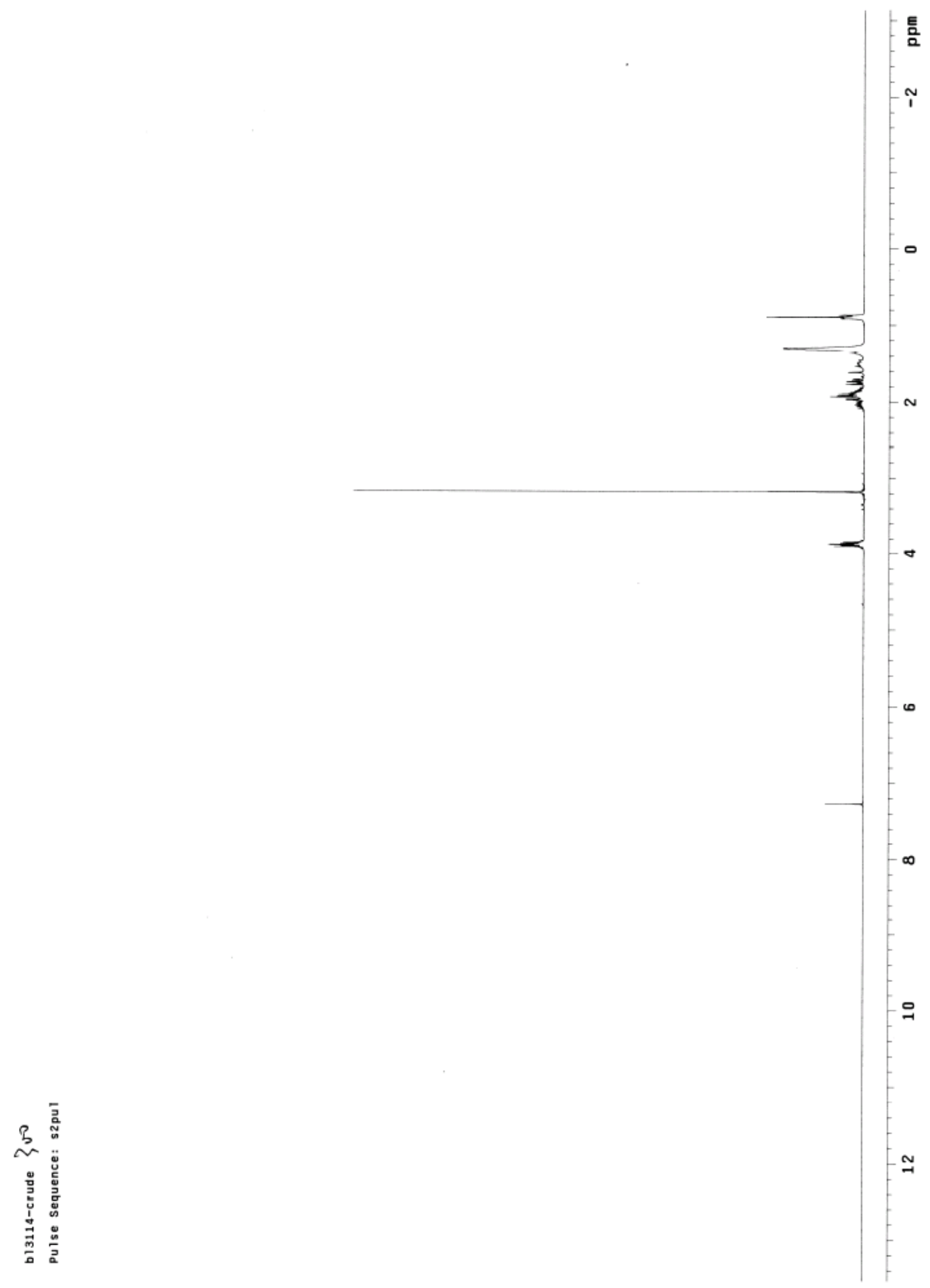


${ }^{13} \mathrm{C}$ NMR for Table 4, entry 9 (75 MHz)

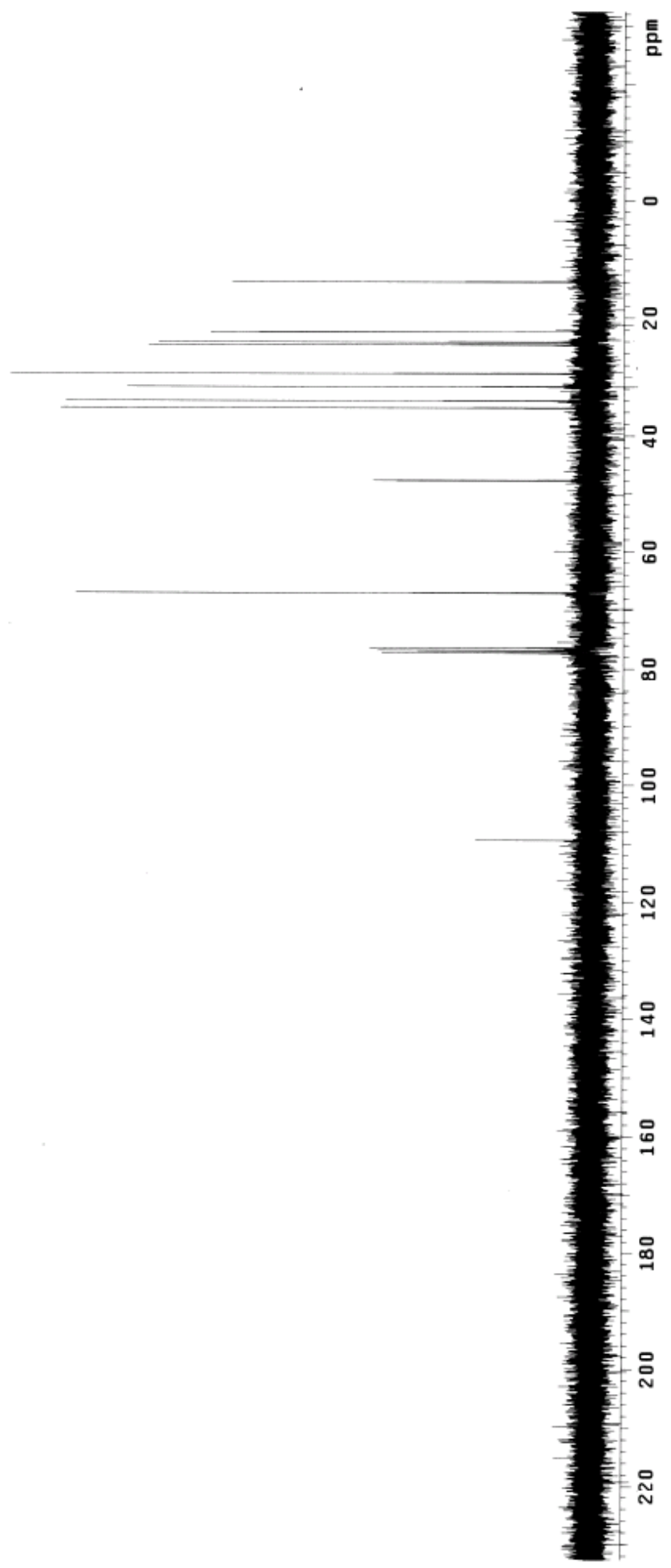


${ }^{1}$ H NMR for Table 4, entry 13 (300 MHz)

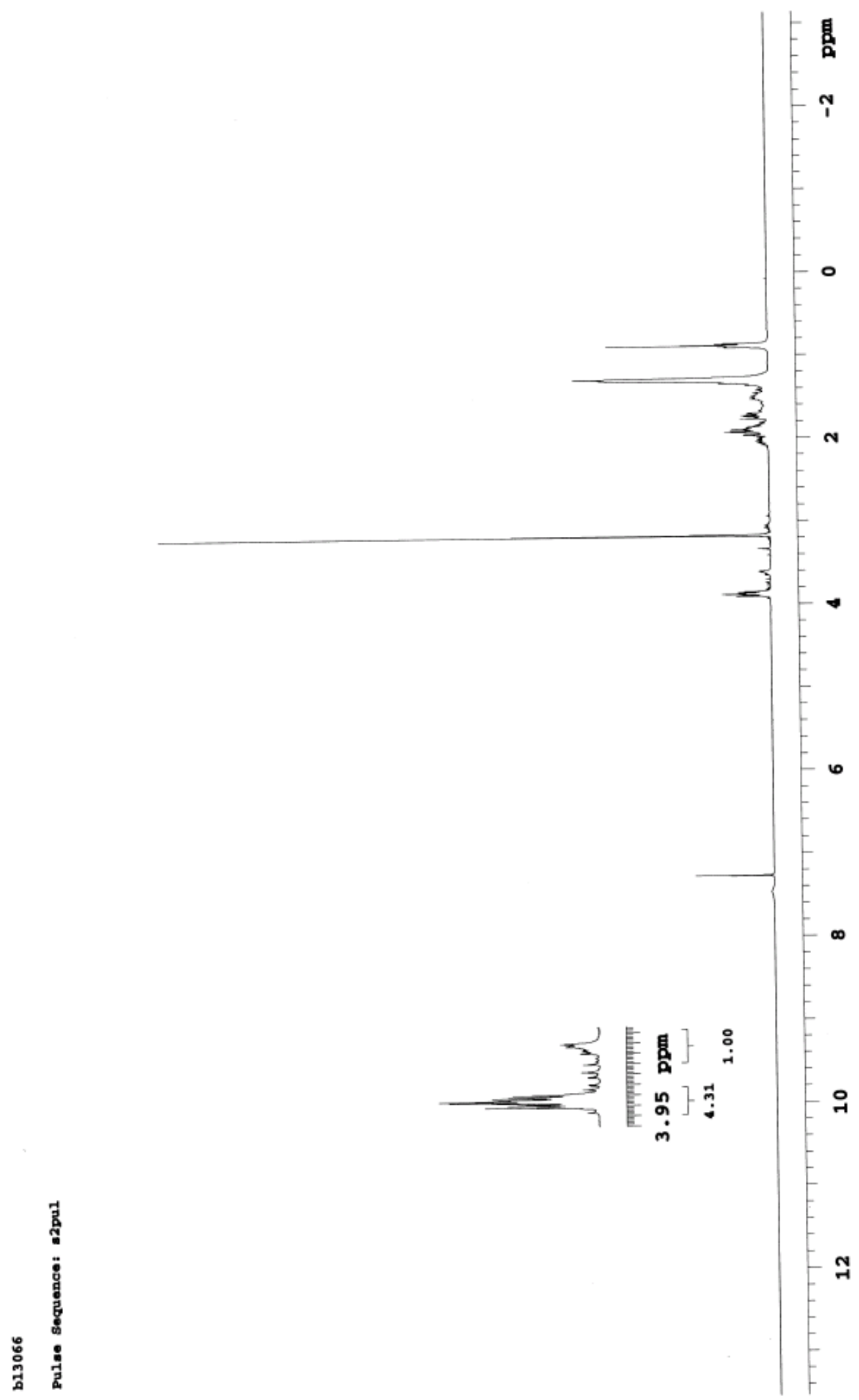



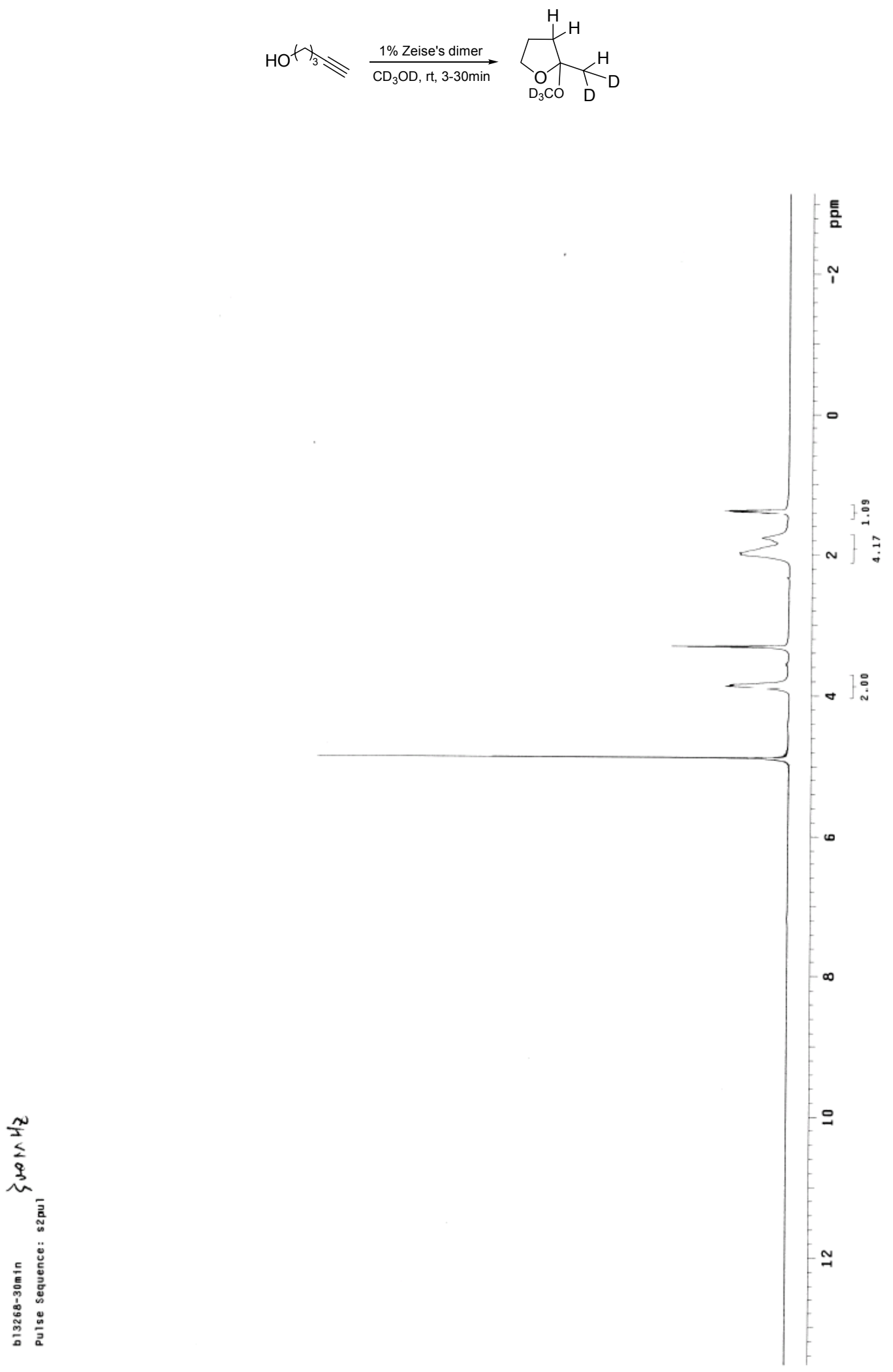


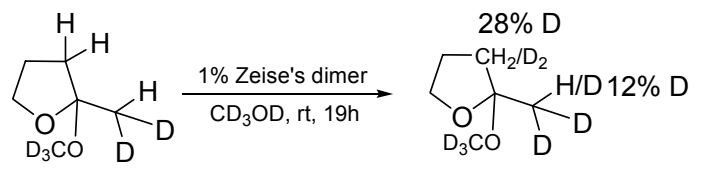

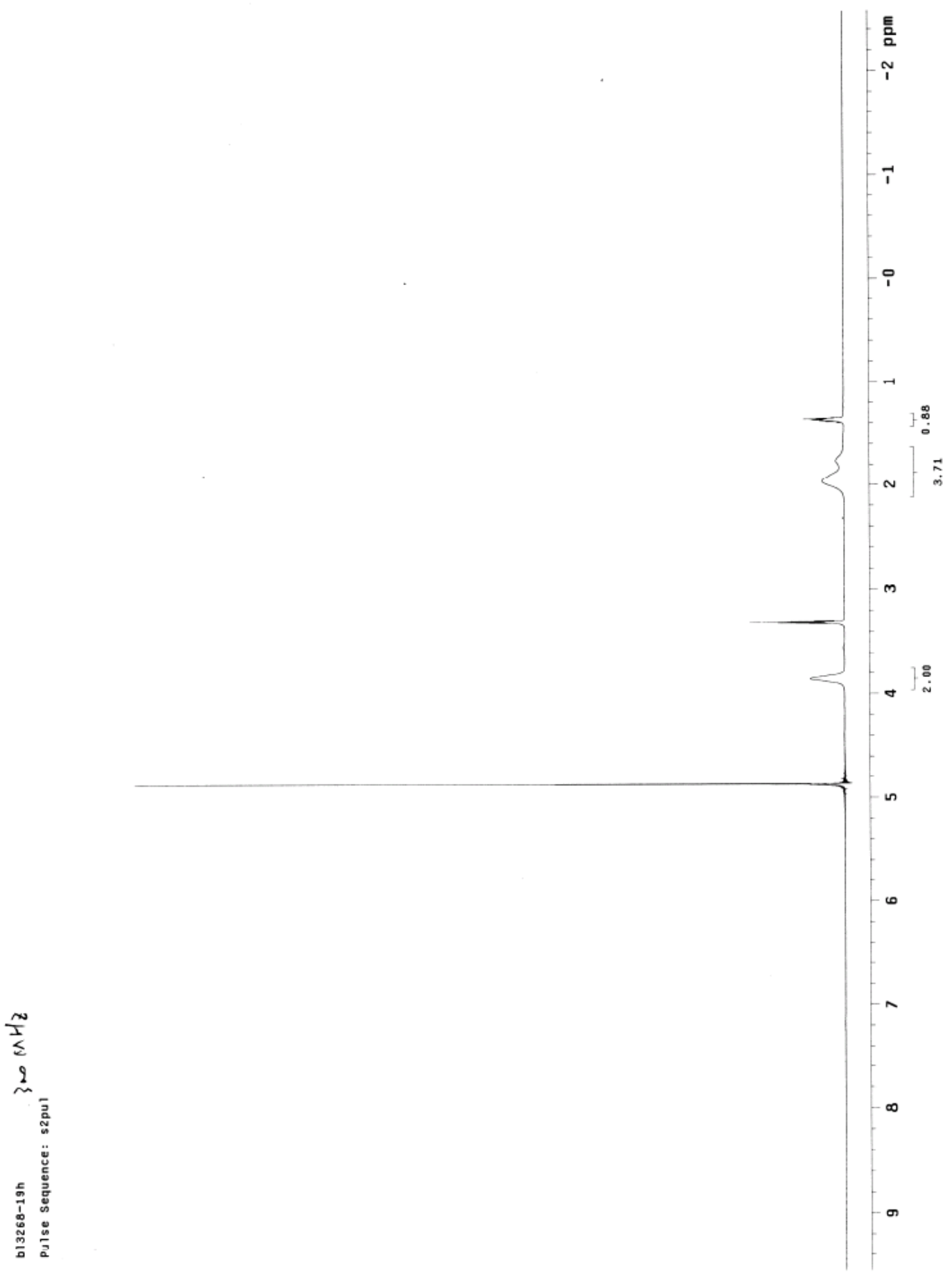

